Network Working Group

Request for Comments: 2564

Category: Standards Track

\author{
C. Kalbfleisch \\ Verio, Inc. \\ C. Krupczak
}

Empire Technologies, Inc.

R. Presuhn

BMC Software, Inc.

J. Saperia

IronBridge Networks

May 1999

\title{
Application Management MIB
}

Status of this Memo

This document specifies an Internet standards track protocol for the Internet community, and requests discussion and suggestions for improvements. Please refer to the current edition of the "Internet Official Protocol Standards" (STD 1) for the standardization state and status of this protocol. Distribution of this memo is unlimited.

Copyright Notice

Copyright (C) The Internet Society (1999). All Rights Reserved.

Abstract

This memo defines a standards track portion of the Management Information Base (MIB) for use with network management protocols in the Internet Community. In particular, it defines objects used for the management of applications. This MIB complements the system Application MIB, providing for the management of applications' common attributes which could not typically be observed without the cooperation of the software being managed.

Table of Contents

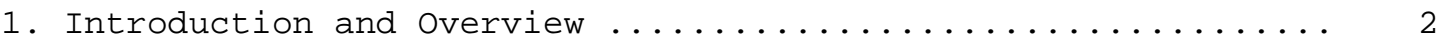

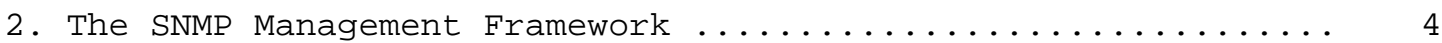

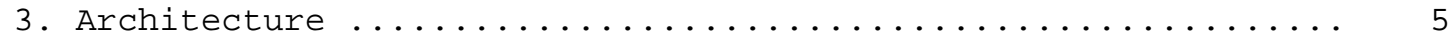

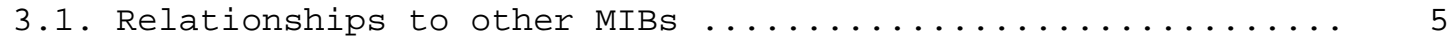

3.1.1. Relationship to the System Application MIB .......... 5

3.1.2. Relationship to the Host Resources MIB .............. 6

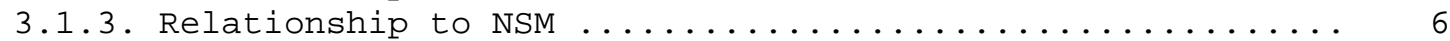

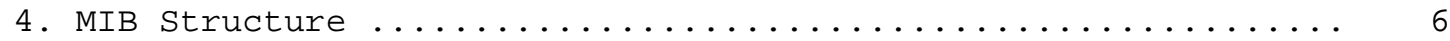

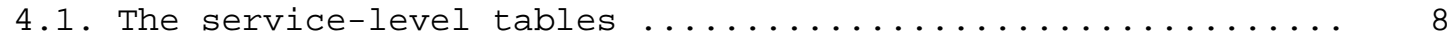

4.1.1. The service name to service instance table ........... 8

4.1.2. The service instance to service name table ........... 9

4.1.3. The service instance to running application element table 9

4.1.4. The running application element to service instance table 9

[Page 1] 


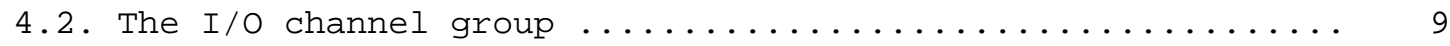

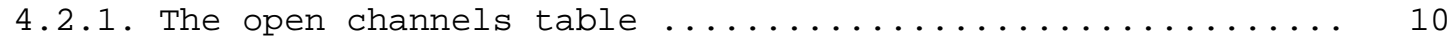

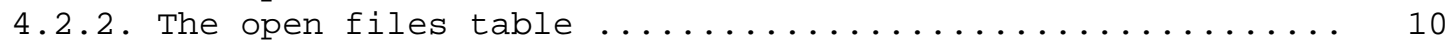

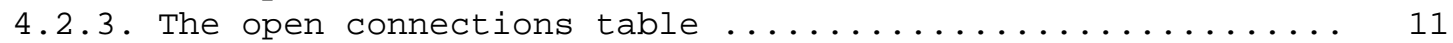

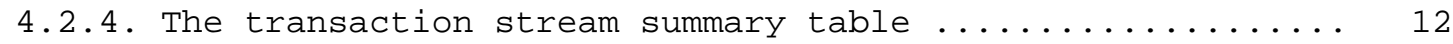

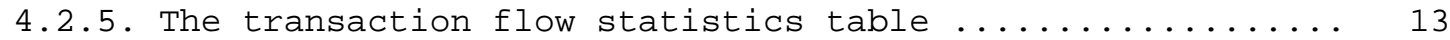

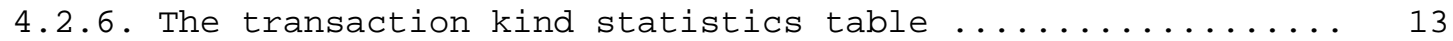

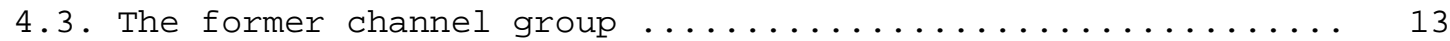

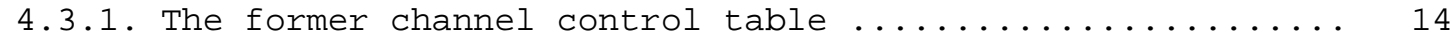

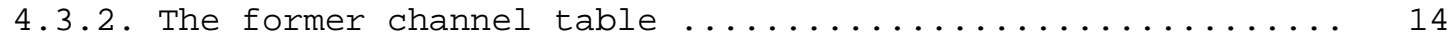

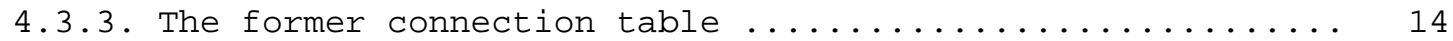

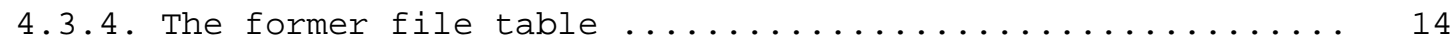

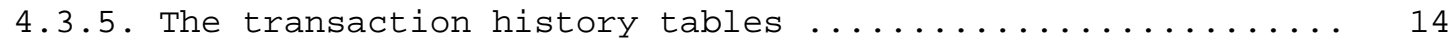

4.4. The running element status and control group ........... 15

4.4.1. The running application element status table ......... 15

4.4.2. The running application element control table ........ 15

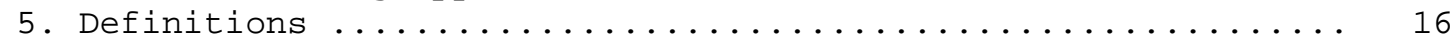

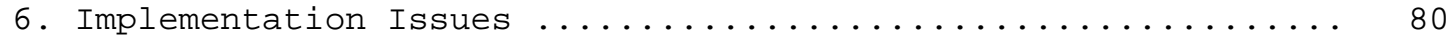

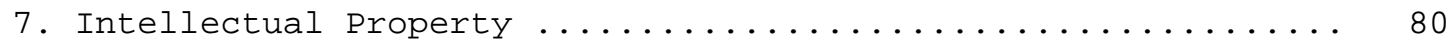

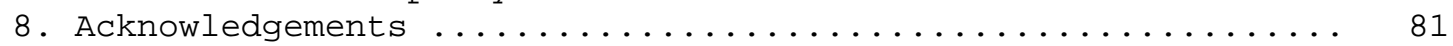

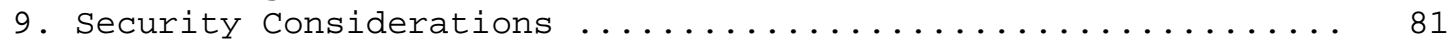

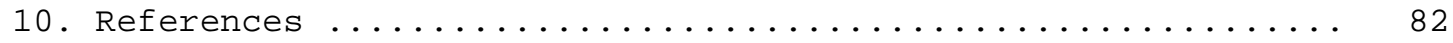

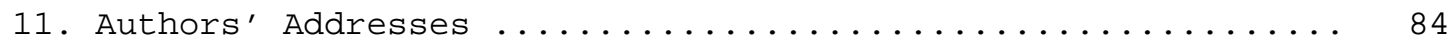

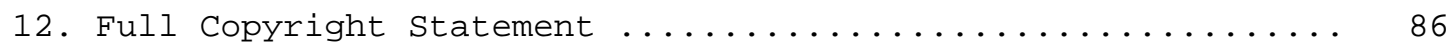

\section{Introduction and Overview}

This document furthers the work begun in the systems application MIB [31].

The development of the "Host Resources MIB" [10], "Network Services Monitoring MIB" [23], "Mail Monitoring MIB" [24], "Relational Database Management System (RDBMS) Management Information Base (MIB) using SMIv2" [12], "Entity MIB using SMIv2" [20], and "Applicability of Standards Track MIBs to Management of World Wide Web Servers" [21] provides us with a base of experience in making a variety of applications visible to management; this specification abstracts out the common aspects of applications management and provides a generic base usable for the management of almost any application.

The key words "MUST", "MUST NOT", "REQUIRED", "SHALL", "SHALL NOT", "SHOULD", "SHOULD NOT", "RECOMMENDED", "MAY", and "OPTIONAL" in this document are to be interpreted as described in RFC 2119 [22] .

Due to the design decision to not require application instrumentation, many important topics were not handled in system application MIB [31]. The following topics are within the scope of this document: 


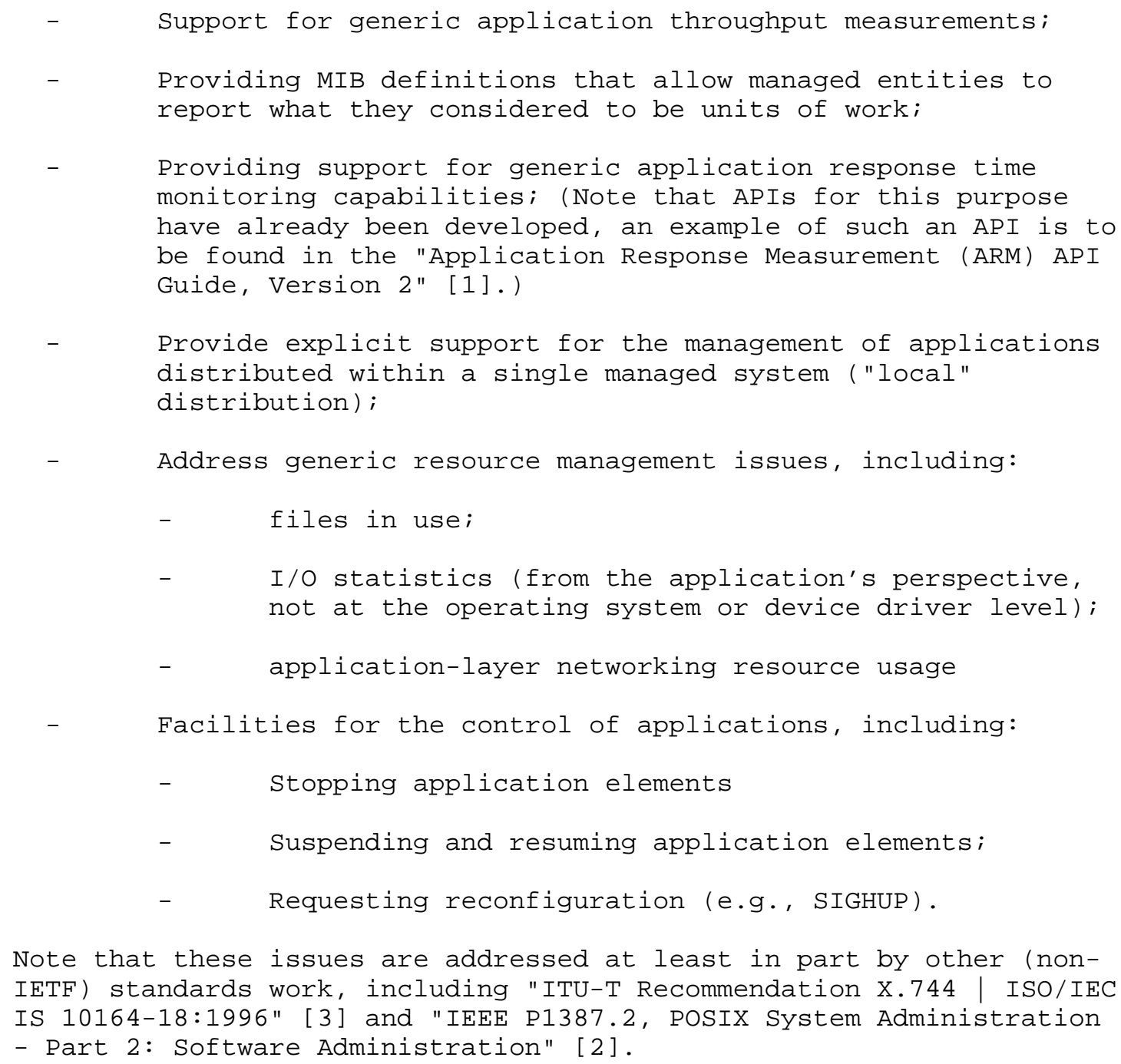


2. The SNMP Management Framework

The SNMP Management Framework presently consists of five major components:

An overall architecture, described in RFC 2571 [26].

Mechanisms for describing and naming objects and events for the purpose of management. The first version of this structure of Management Information (SMI) is called SMIV1 and described in STD 16, RFC 1155 [4], STD 16, RFC 1212 [6] and RFC 1215 [7]. The second version, called SMIv2, is described in STD 58, RFC 2578 [15], RFC 2579 [16] and RFC 2580 [17].

Message protocols for transferring management information. The first version of the SNMP message protocol is called SNMPv1 and described in STD 15, RFC 1157 [5]. A second version of the SNMP message protocol, which is not an Internet standards track protocol, is called SNMPV2c and described in RFC 1901 [14] and RFC 1906 [19]. The third version of the message protocol is called SNMPv3 and described in RFC 1906 [19], RFC 2572 [27] and RFC 2574 [29].

Protocol operations for accessing management information. The first set of protocol operations and associated PDU formats is described in STD 15, RFC 1157 [5]. A second set of protocol operations and associated PDU formats is described in RFC 1905 [18].

A set of fundamental applications described in RFC 2573 [28] and the view-based access control mechanism described in RFC 2575 [30].

Managed objects are accessed via a virtual information store, termed the Management Information Base or MIB. Objects in the MIB are defined using the mechanisms defined in the SMI.

This memo specifies a MIB module that is compliant to the SMIv2. A MIB conforming to the SMIv1 can be produced through the appropriate translations. The resulting translated MIB must be semantically equivalent, except where objects or events are omitted because no translation is possible (use of Counter64). Some machine readable information in SMIV2 will be converted into textual descriptions in SMIv1 during the translation process. However, this loss of machine readable information is not considered to change the semantics of the MIB. 


\section{Architecture}

Object-oriented modeling techniques like subclassing and multiple inheritance can be emulated in the SNMP information model through the use of tables with common indexes.

The challenge for the developer of management applications is to recognize those situations in which various aspects of a single logical resource are represented in several different tables, possibly defined in different MIBs.

Most of the management information defined here may pertain to any number of applications in a managed system. The simplest way of supporting this requirement within the SNMP information model is to use tables. This means that the management information for a particular resource may be found in one or more rows of one or more tables; the fact that this information pertains to a single resource may be inferred from the index values used, possibly with the support of mapping tables. This also means that a single table may contain management information relevant to a number of applications. This has significant implementation implications; see the implementation issues section below for more information.

\subsection{Relationships to other MIBs}

This section outlines the relationships of the components of this MIB (usually in the form of common indexing structures) to:

$$
\begin{aligned}
& \text { - } \quad \text { the systems applications MIB [31] } \\
& \text { - } \quad \text { the host resources MIB [10] } \\
& \text { - } \quad \text { the network services monitoring MIB [23] }
\end{aligned}
$$

\subsubsection{Relationship to the System Application MIB}

The system application MIB defines attributes for management of applications which can be realized without instrumenting the application itself. This specification extends that framework to include additional attributes which will typically require instrumentation within the managed resource. The sysApplRunElmtIndex is the key connection between these two MIBs; it is essential that implementations of this MIB and of the system applications MIB running concurrently on a given platform employ a consistent policy for assigning this value to identify running application elements. 


\subsubsection{Relationship to the Host Resources MIB}

The Host Resources MIB [10] supplies information on the hardware, operating system, installed and running software on a host.

The Host Resources MIB has three hardware groups ("hrsystem", "hrStorage" and "hrDevice") and three software groups ("hrSwRun", "hrSWRunPerf" and "hrSWInstalled"). Of these, the software groups are of greatest significance to this MIB.

The software groups define management information on the software used in the system. The information provided is grouped into (1) the currently running, (2) the performance and (3) the installed applications.

The index "hrSWRunIndex" used in the "hrSWRunTable" and other tables to identify running software by process identifier (or equivalent) relates information in the Host Resources MIB to information in the System Applications MIB and this MIB. It is essential that the values assigned to hrSWRunIndex from the Host Resources MIB be consistent with the values used for sysApplRunElmtIndex.

\subsubsection{Relationship to NSM}

The Network Services Monitoring MIB [23] is defined as the base set of attributes for managing network applications. The Application MIB includes information normally obtainable only from the managed resource itself, rather than the supporting system. Due to differences in index representation, the relationship between the Network Services Monitoring MIB and the Application MIB is not formally defined.

\section{MIB structure}

This MIB is organized into several groups, which in turn are organized into tables to provide the monitoring and control of information relevant to the management of applications. The groups model:

- $\quad$ the service-level view of applications
- $\quad \begin{aligned} & \text { information on open channels (files, connections, } \\ & \text { transaction streams) in use by applications }\end{aligned}$
- $\quad$ historical information on former channels
- $\quad$ process-level status and control information

Kalbfleisch, et al. Standards Track [Page 6] 
These groups are organized into various tables. Information for a particular running managed application appears in the form of entries in the appropriate tables. The tables are:

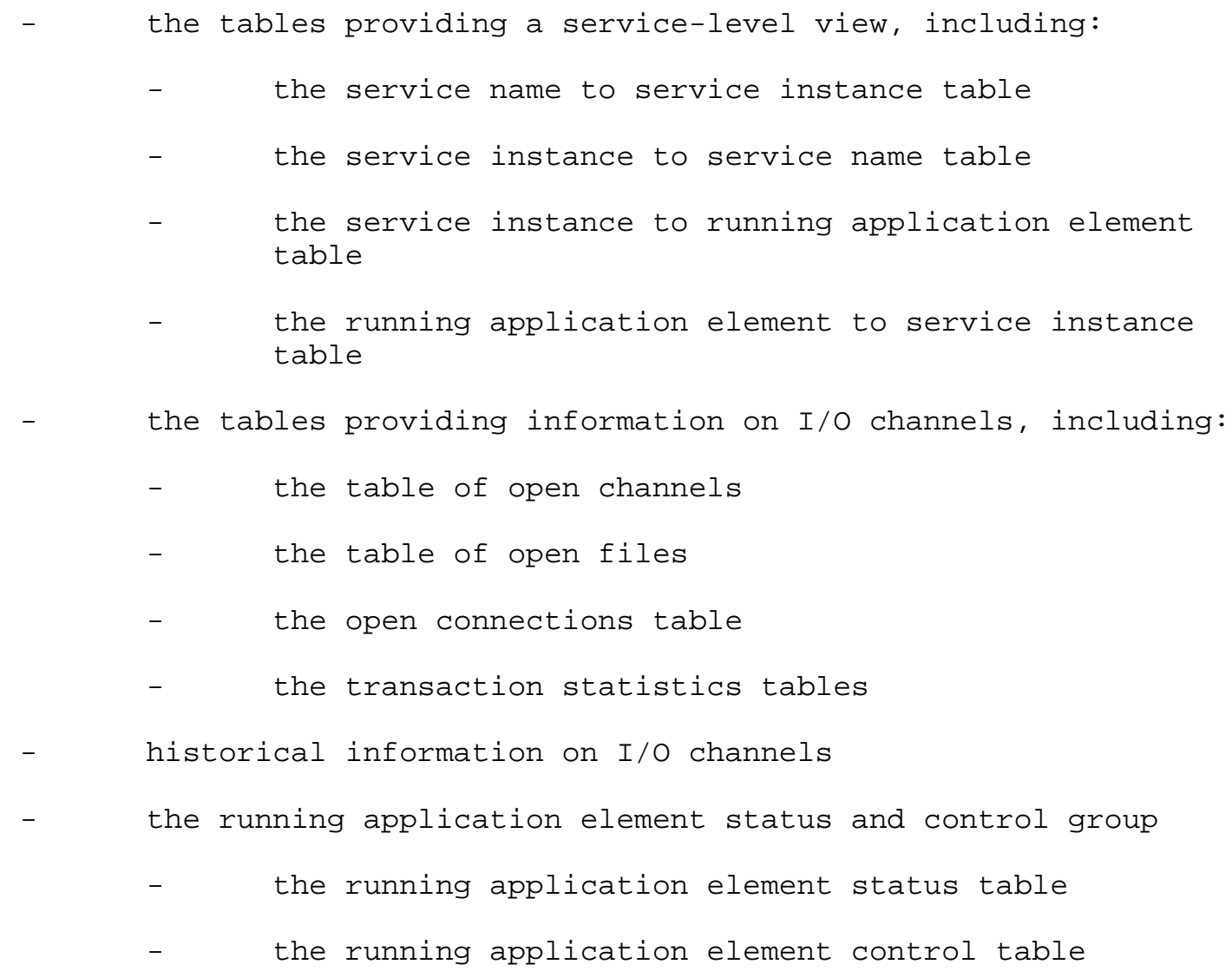


the relevant DESCRIPTION clauses. Note that a discontinuity in one of these counters does not imply a sysUpTime.0 discontinuity, nor does a sysuptime.0 discontinuity imply a discontinuity in any of these counters.

4.1. The service-level tables

The service-level tables permit the identification of one or more instances of named services on a system, and the association of running application elements to these services.

Service names are represented as human-readable strings, using values assigned by IANA where possible. The allocation of unique values for service instance identifiers is a local administrative issue; the values allocated must be constant for the lifetime of the service instance, and re-use of values should be avoided.

It is important to understand that a service is not the same thing as a protocol. Rather, some services may be at least partially described by the protocol(s) used to provide that service.

In deciding what should or should not be considered a service, the following factors merit consideration:

- is there an identifiable set of resources associated with providing this service?

- is there a reasonably long-lived server or client process?

Following this reasoning, one can see where SMTP and HTTP service providers would be good candidates for classification as services for purposes of application management, where finger probably would not. Of course, implementors of this MIB are free to define additional services. An applicability statement may be an appropriate vehicle for standardizing how a specific service's information is reported using this MIB.

4.1.1. The service name to service instance table

The service name to service instance table uses the service name as its primary key, and the service instance identifier as its secondary key. It facilitates the identification and lookup of the instances of a given service in a system. 


\subsubsection{The service instance to service name table}

The service instance to service name table uses the service instance identifier as its primary key, and the service name as its secondary key. Given a service instance identifier, it facilitates the lookup of the name of the service being provided.

4.1.3. The service instance to running application element table

The service instance to running application element table uses the service instance identifier as its primary key, and the running application element index as its secondary key. This facilitates the identification of the set of running application elements providing a given instance of a service.

4.1.4. The running application element to service instance table

The running application element to service instance table uses the running application element index as its primary key and the service instance identifier as its secondary key. It identifies the set of services provided by a given running application element.

4.2. The I/O channel group

Information processed by an application can be modeled using the concept of a channel. Two kinds of channels, for example, are files and network connections.

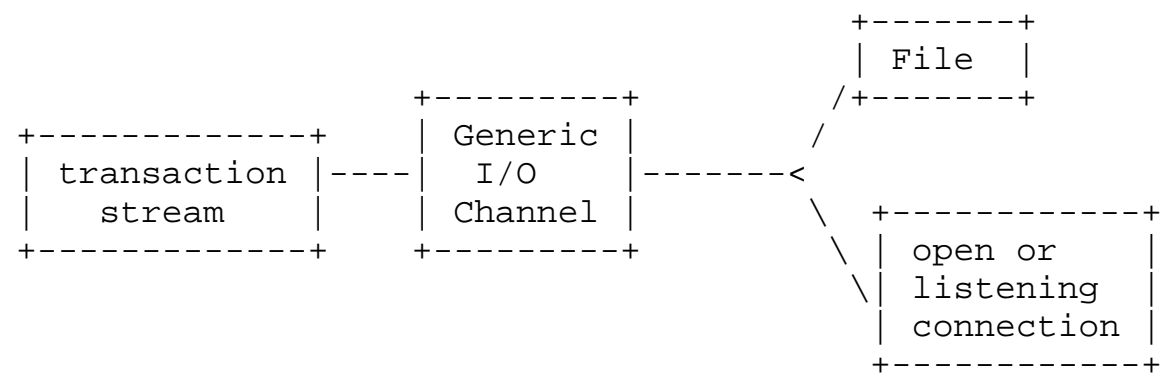

For each entry in the open channel table, there will be a corresponding entry in either the open file table or the open connection table.

The information flowing on a channel may be structured as transactions. When the information flow on a channel is being monitored as a transaction stream, an entry in the transaction stream table will represent this fact and the associated information about 
that stream.

To facilitate traversal of these tables and retrieval of information relevant to a specific running application element or service instances, the initial indexes of these tables are the same. In each case, the first index determines whether the second index is interpreted as a running application element identifier or as a service instance identifier. The third index serves to uniquely identify a channel (and consequently, an open connection or file) in the context of a running application element or service instance.

The transaction stream summary table contains per-stream summaries of transaction statistics. The transaction flow statistics table contains statistics broken into both transmit and receive counts for requests and responses on each stream. The transaction kind statistics table contains information further broken down by transaction kind.

The transaction tables have a common structure for their indexing, with additional indexes added for increasing detail. The initial three indexes are the same as all the other tables in this group, serving to uniquely identify each transaction stream.

4.2.1. The open channels table

The following information is available in this table:

- time at which the channel was opened

- number of read requests

- number of bytes read

- time at which most recent read operation was initiated

- number of write requests

- number of bytes written

- time at which most recent write operation was initiated

\subsubsection{The open files table}

The open files table contains one entry for each file in use by a manageable running application element. (See "Definitions of System-Level Managed Objects for Applications" [31] for a detailed definition of a running application element.) The purpose of this table is to identify the files in use and to record information 


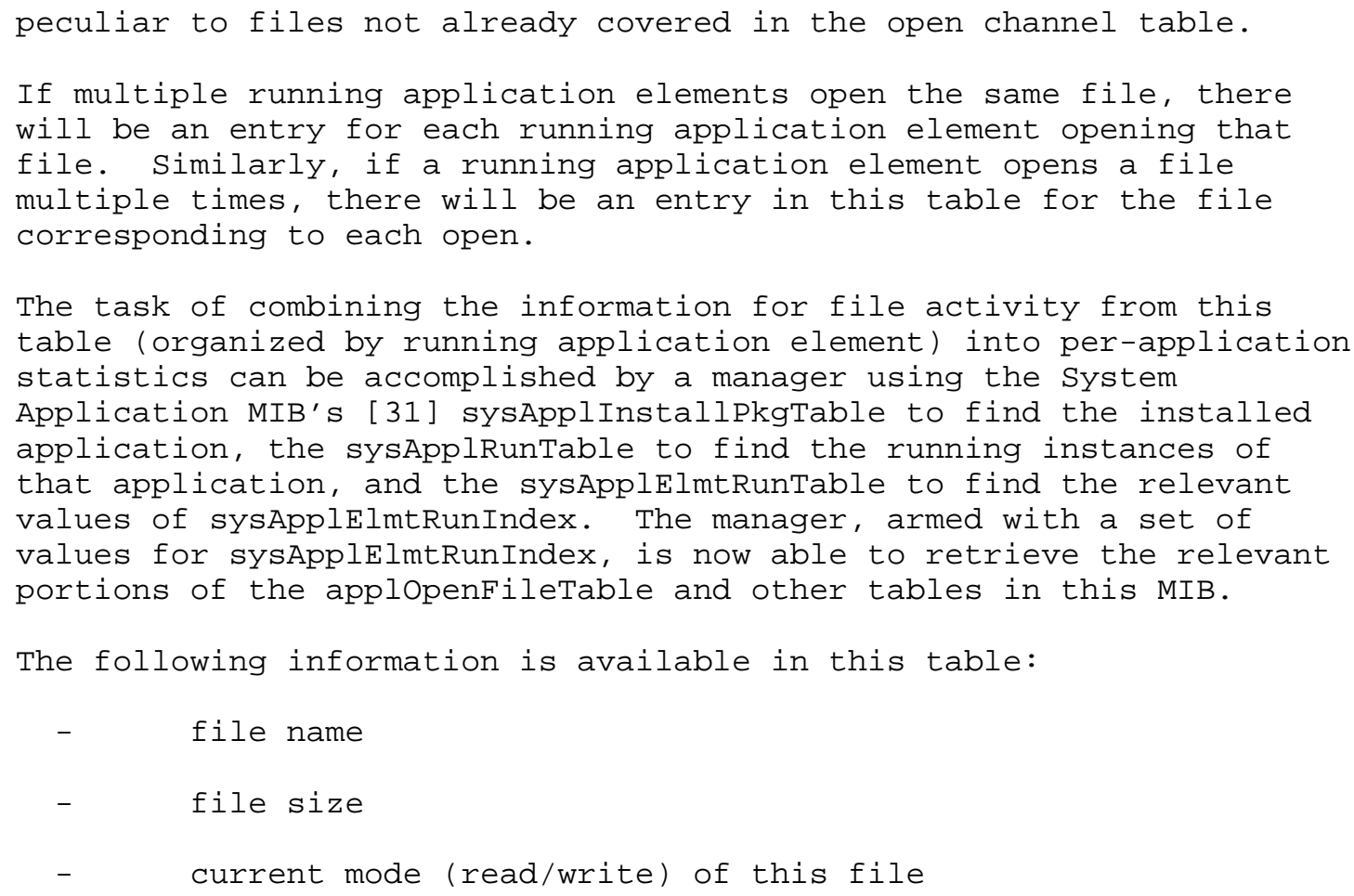

By convention, the names "stdin", "stdout" and "stderr" are used when these streams cannot be resolved to actual file names.

\subsubsection{The open connections table}

This table provides information on channels that are open connections or listeners.

The following information is available for each connection:

- identification of the transport protocol in use

- near-end address and port

- far-end address and port

- identification of the application layer protocol in use 


\subsubsection{The transaction stream summary table}

The transaction stream summary table contains per-stream summaries of transaction statistics. The simple model of a transaction used here looks like this:

invoker $\left|\begin{array}{c|}\text { Request } \\ -----> \\ \text { Response } \\ <------\end{array}\right|$ performer

Since in some protocols it is possible for an entity to take on both the invoker and performer roles, information here is accumulated for transmitted and received requests, as well as for transmitted and received responses. Counts are maintained for both transactions and bytes transferred. The information represented in this table includes:

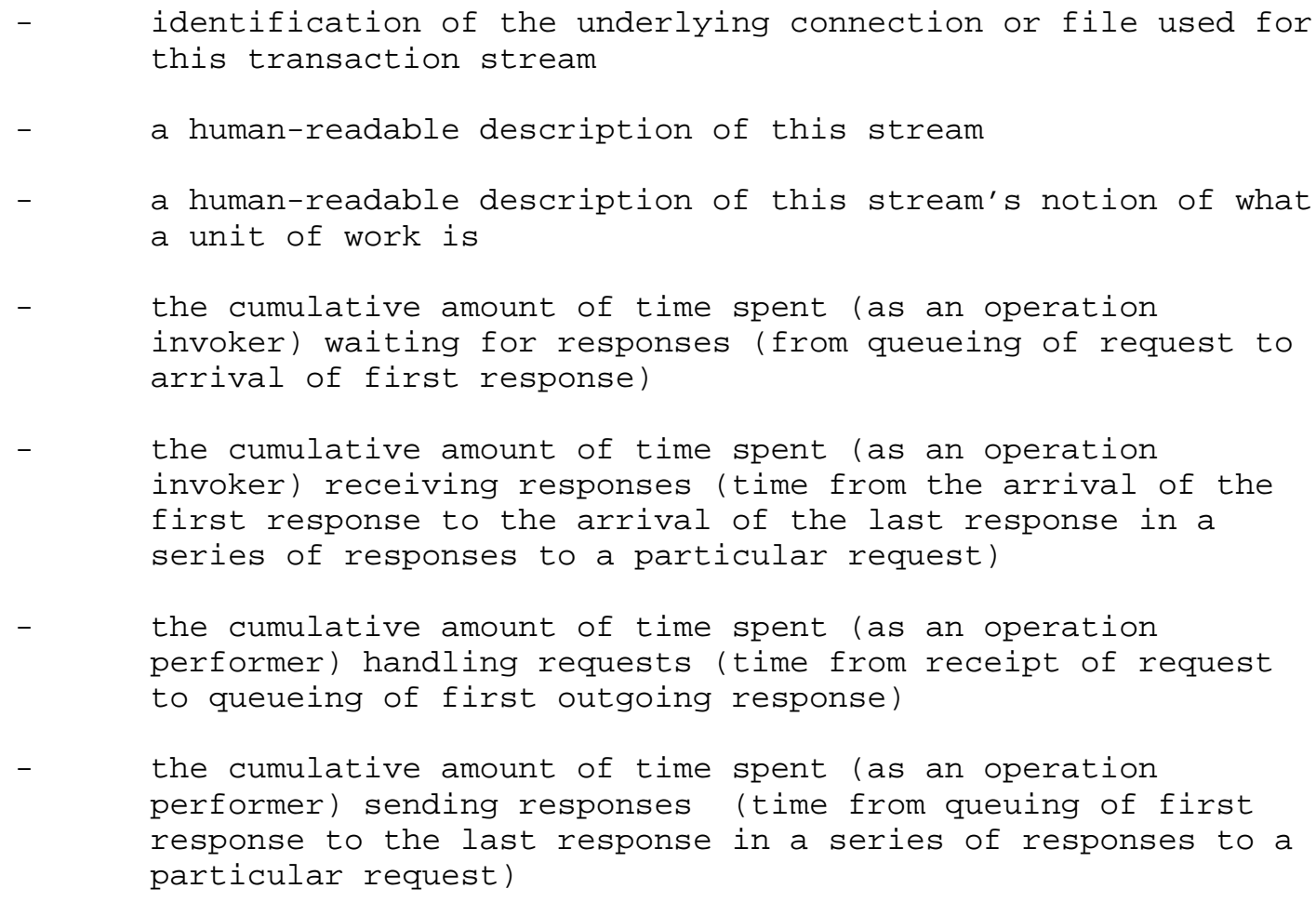




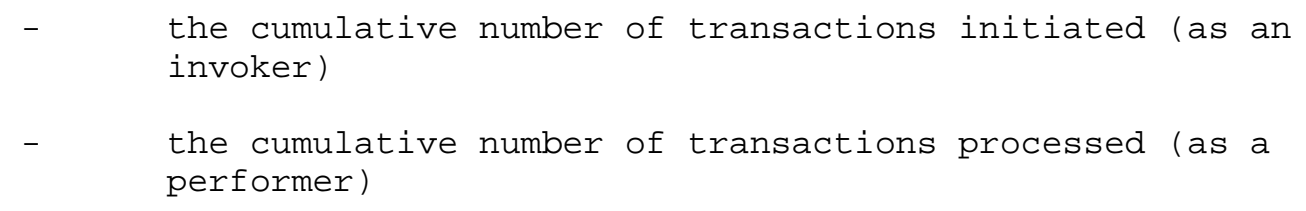

4.3. The former channel group

The former channel group has several tables. The former channel control table controls the retention of history information by a running application element or service instance. The remaining tables parallel the structure of the channel group, with one significant difference in indexing structure. The closed channel index is independent from the open channel index. 


\subsubsection{The former channel control table}

The former channel control table provides control over the accumulation of information on former connections for running application elements and service instances. For each one, this table, indexed by the running application element or service instance index, controls whether information on former channels is accumulated, how many of these history records are retained, how long these are retained (within the lifetime of the process), and a count of history entries that were deleted before their expiration time in order to make room for new entries.

\subsubsection{The former channel table}

The former channel table provides historical information on channels that have been closed. The number and lifetime of these entries is controlled, for each running application element or service instance, by the former channel control table. Most of the information in this table corresponds to information in the open channel table.

For the connection or file-specific aspects of a given former channel, an entry will exist in the former connection table or in the former file table.

\subsubsection{The former connection table}

For formerly open channels that were connections, connection-specific historical information is kept in the former connection table. For each entry in the former connection table, there will be an identically indexed entry in the former channel table.

\subsubsection{The former file table}

For formerly open channels that were files, file-specific historical information is kept in the former file table. For each entry in the former file table, there will be an identically indexed entry in the former channel table.

\subsubsection{The transaction history tables}

Two tables provide per-transaction-kind breakdowns for channels carrying transaction-structured flows. These tables are analogous to the transaction flow and kind statistics tables, with similar index structures. 
4.4. The running element status and control group

The running application element status and control group has two tables.

4.4.1. The running application element status table

This table provides information for a running application element. Indexed by the sysApplelmtRunIndex, an entry in this table reports useful information on that running element's resource usage. Entries in this table contain:

- current heap usage for this running application element

- current number of open network connections for this running application element

- the most recent error status message issued by this running application element

Note that other information, such as the current number of open files for this running application element, is available from the sysapplelmtRunTable in [31].

4.4.2. The running application element control table

This table provides rudimentary control over a running application element. Indexed by the sysApplelmtRunIndex, an entry in this table gives a manager with appropriate permissions the ability to suspend and resume processing by this running element, the ability to request reconfiguration, and the ability to terminate the running element.

Variables in this table include:

- a suspend/resume control

- a reconfiguration request control

- a termination request control 
5. Definitions

APPLICATION-MIB DEFINITIONS : := BEGIN

IMPORTS

MODULE-IDENTITY, OBJECT-TYPE, Counter64, Counter32, Gauge32, mib-2, Unsigned32, zeroDotZero

FROM SNMPV2-SMI

DateAndTime, TEXTUAL-CONVENTION, TestAndIncr, TDomain, Timestamp, TruthValue FROM SNMPV2-TC

SnmpAdminString

FROM SNMP-FRAMEWORK-MIB

MODULE-COMPLIANCE, OBJECT-GROUP

FROM SNMPV2-CONF

LongUtf8String, sysApplElmtRunIndex FROM SYSAPPL-MIB;

applicationMib MODULE-IDENTITY

LAST-UPDATED "9811171815Z"

ORGANIZATION "Application MIB Working Group"

CONTACT-INFO

"http://www. ietf.org/html. charters/applmib-charter.html

Randy Presuhn

BMC Software, Inc.

965 Stewart Drive

Sunnyvale, CA 94086

USA

Telephone: +1 408 616-3100

Facsimile: +1 408 616-3101

EMail: randy_presuhnebmc.com

"

DESCRIPTION

"This MIB defines objects representing generic aspects of applications that are of interest to management but typically require instrumentation within managed application elements.

"

$::=\{\operatorname{mib}-262\}$

$--$

-- $\quad$ Registration hierarchy for this MIB

$--$

applicationMibobjects OBJECT IDENTIFIER : :=

\{ applicationMib 1 \}

Kalbfleisch, et al.

Standards Track

[Page 16] 


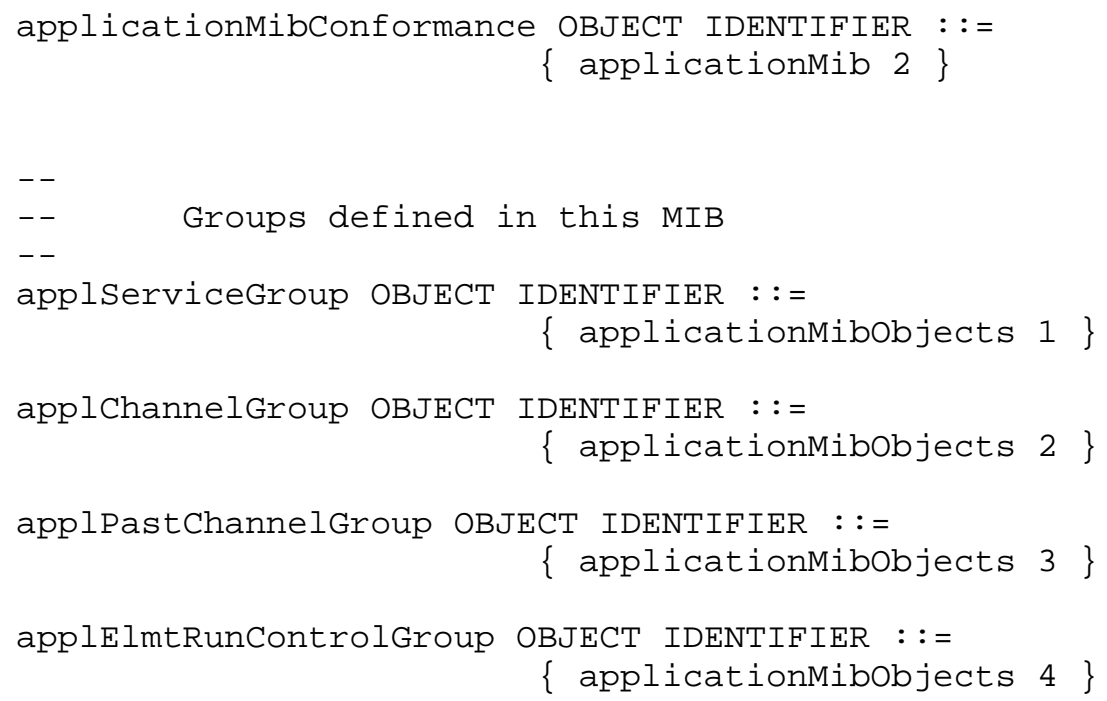




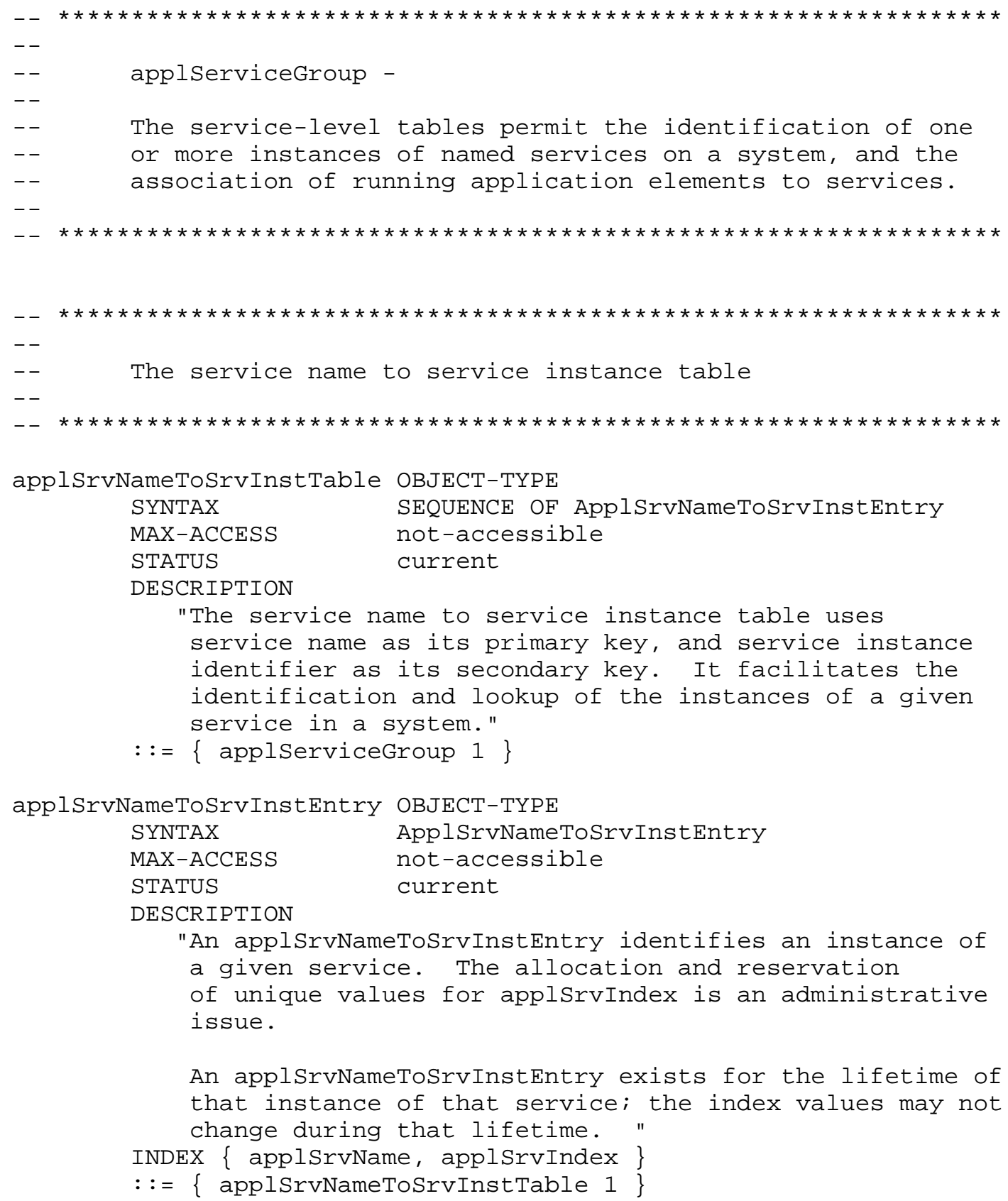




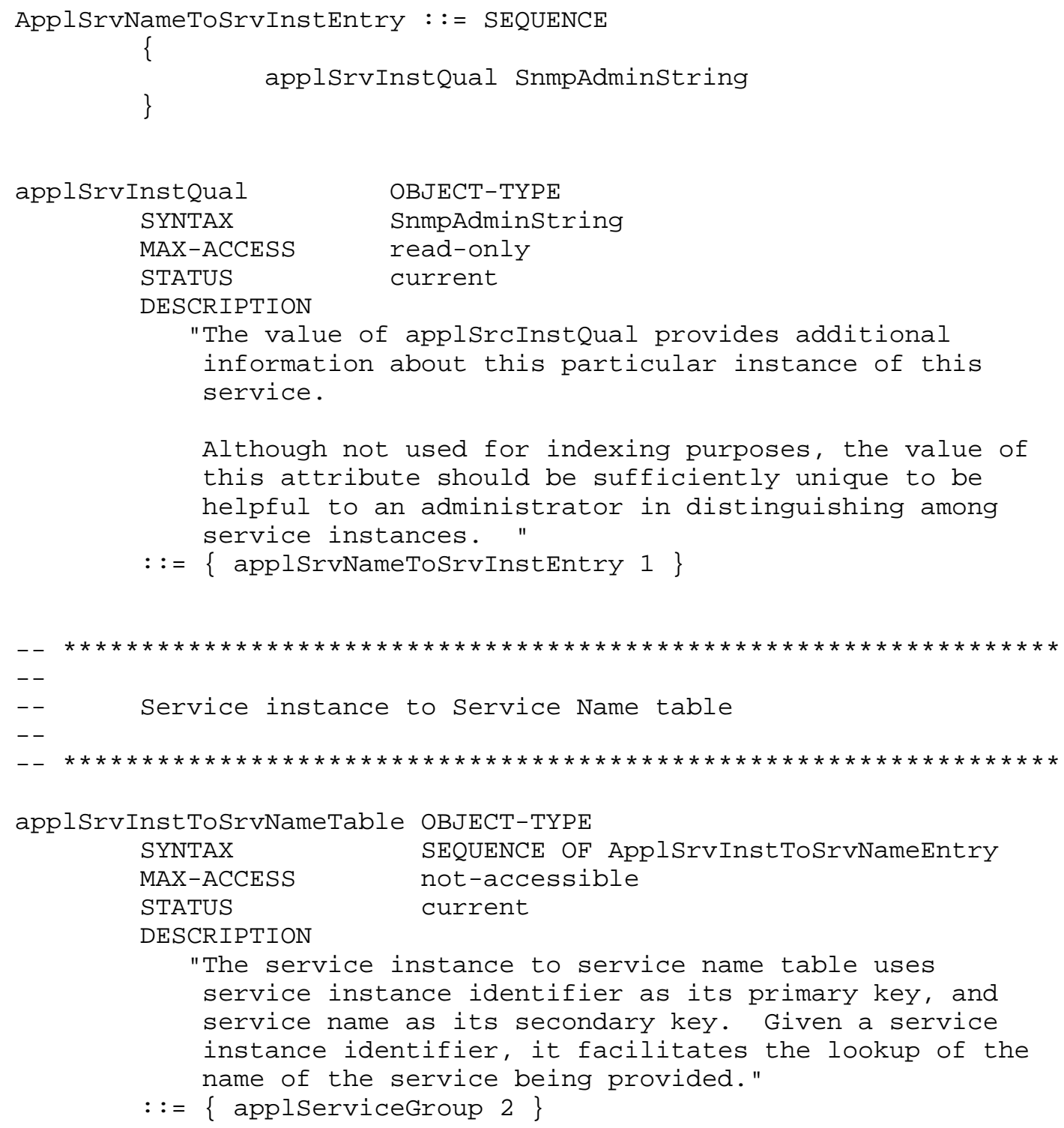




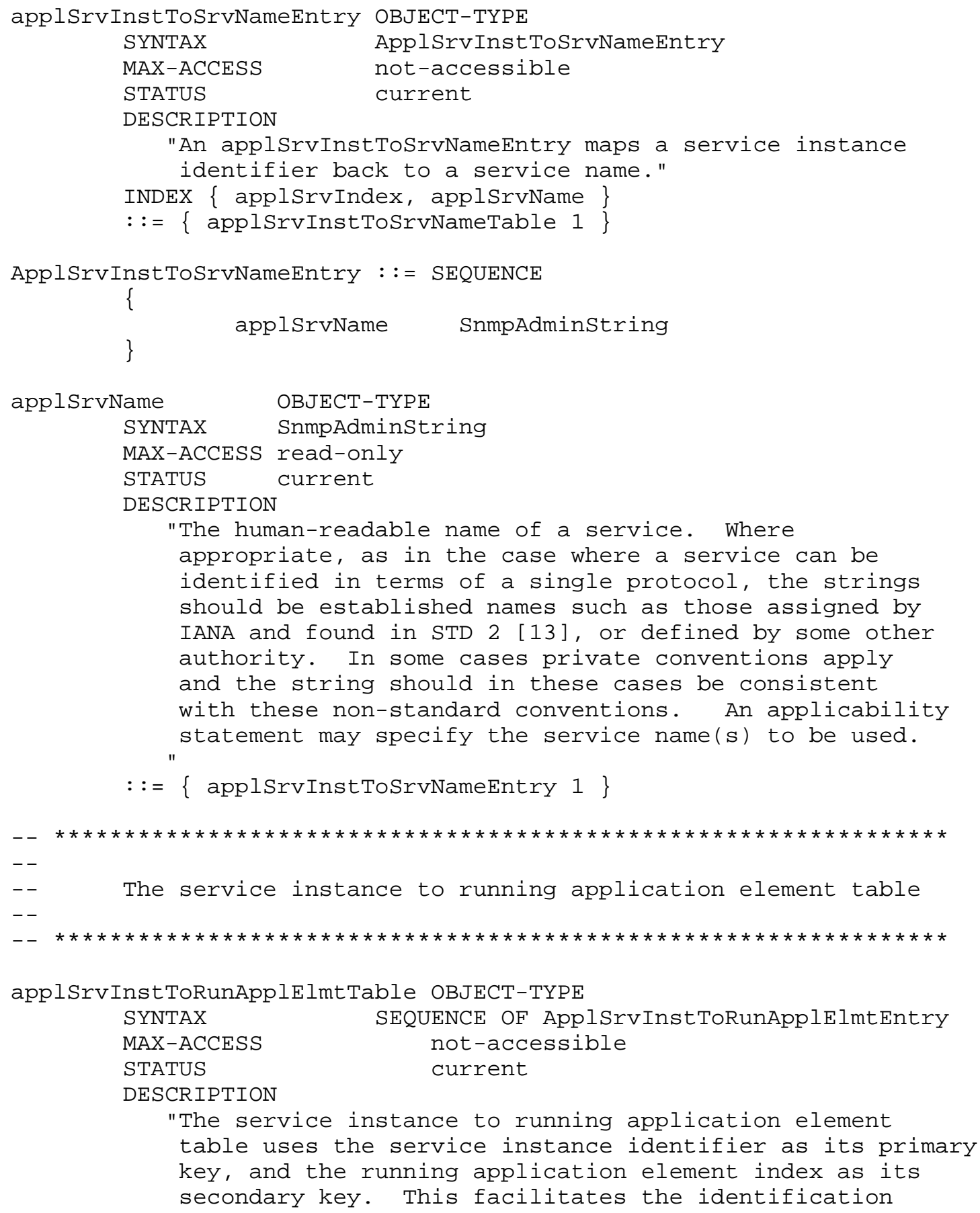




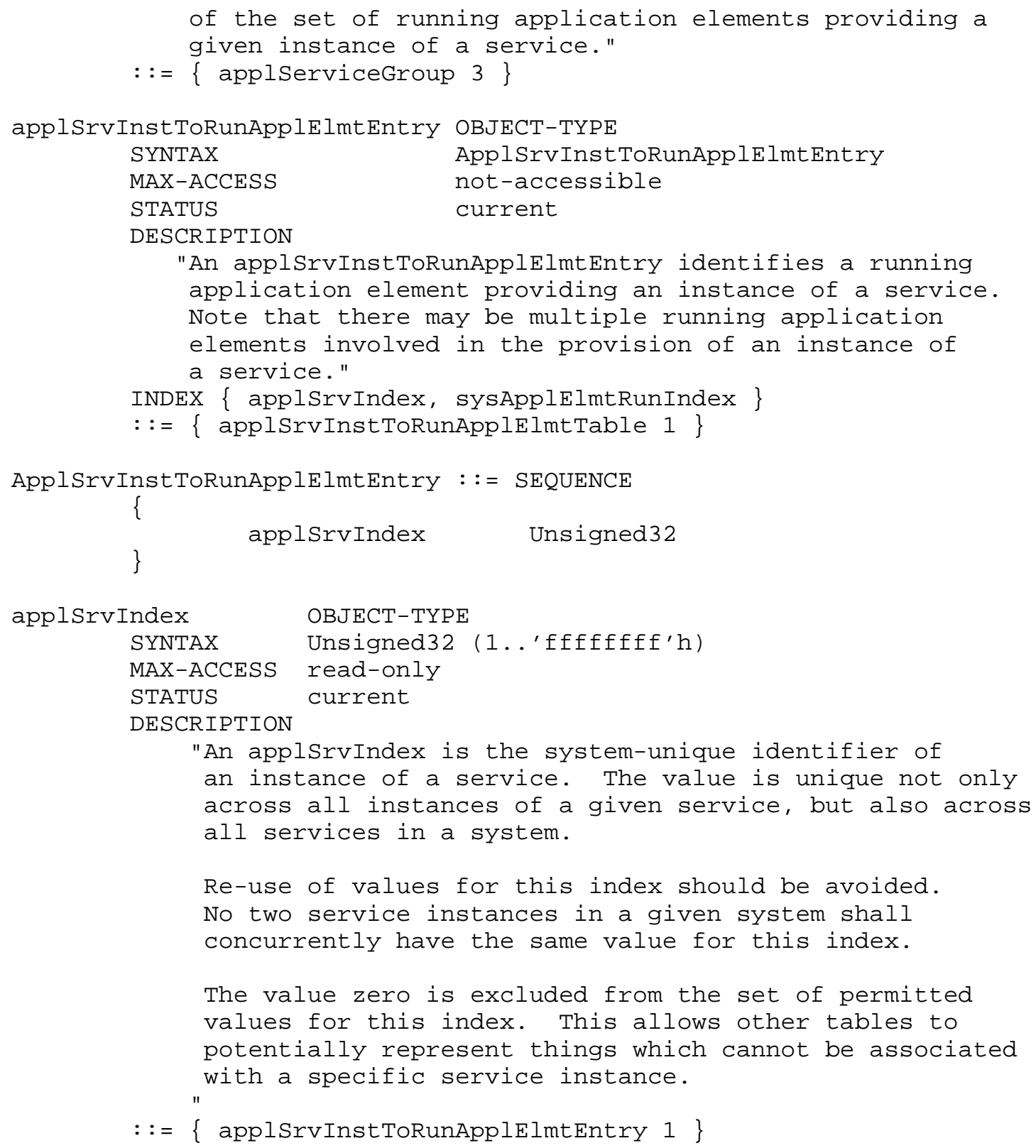




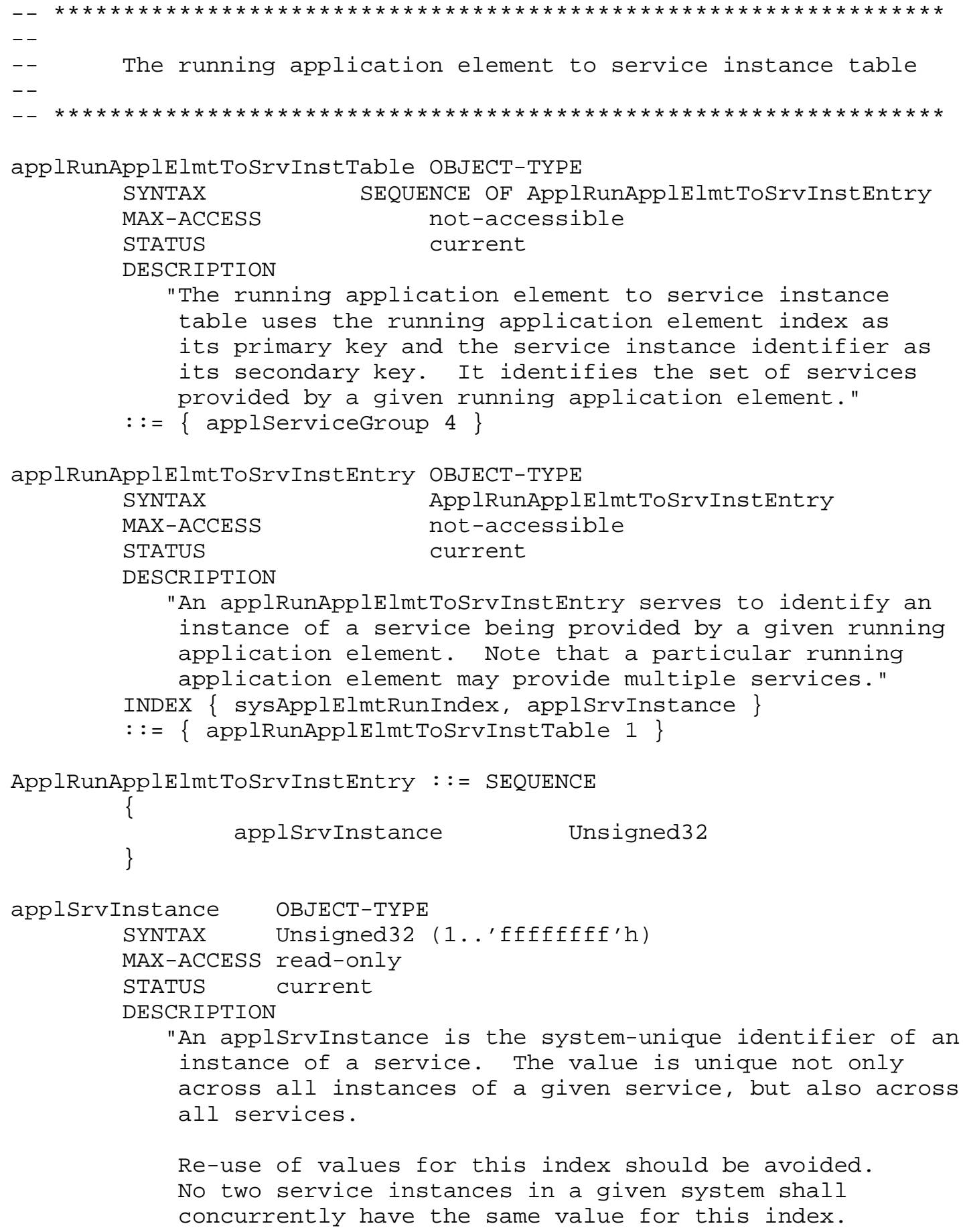


The value zero is excluded from the set of permitted values for this index. This allows other tables to potentially represent things which cannot be associated with a specific service instance.

This attribute is semantically identical to applSrvIndex."

$::=\{$ applRunApplelmtToSrvInstEntry 1$\}$
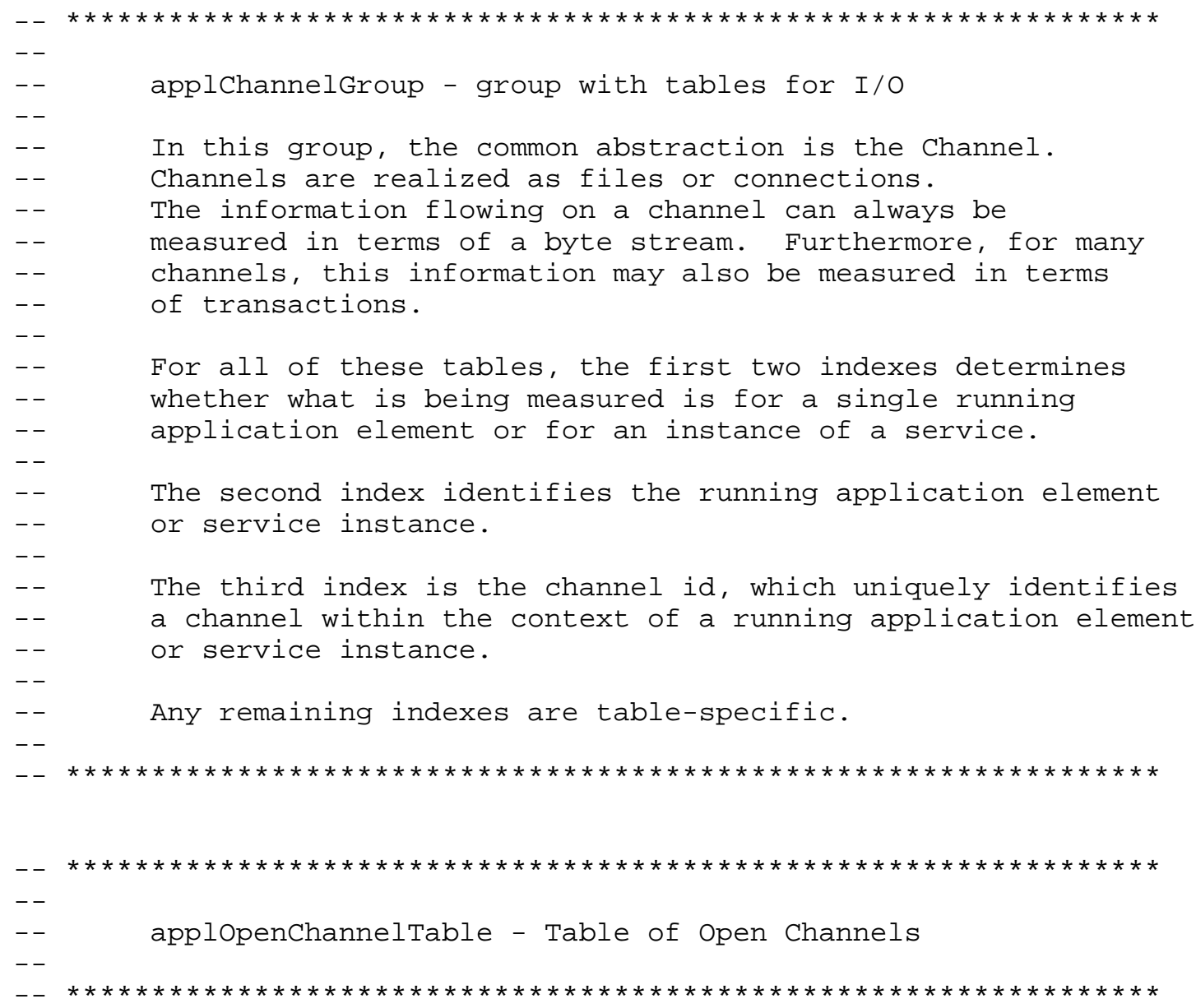

applopenChannelTable OBJECT-TYPE

SYNTAX SEQUENCE OF ApplOpenChannelEntry

MAX-ACCESS not-accessible

STATUS current

[Page 23] 
DESCRIPTION

"The applopenchannelTable reports information on open channels for running application elements and for service instances. This table is indexed by applElmtOrSvc, applElmtOrSvcId, and applopenchannelIndex. This effectively groups all entries for a given running application element or service instance together. Applchannelindex uniquely identifies an open channel (and, consequently, a file or connection) within the context of a particular running application element or service instance.

Some of the information in this table is available through both sixty-four and thirty-two bit counters. The sixty-four bit counters are not accessible in protocols that do not support this data type." $::=\{$ applChannelGroup 1$\}$

applOpenChannelEntry OBJECT-TYPE

SYNTAX ApplOpenChannelEntry

MAX-ACCESS not-accessible

STATUS current

DESCRIPTION

"An applopenchannelEntry indicates that a channel has been opened by this running application element or service instance and is still open. Note that if a file has been opened multiple times, even by the same process, it will have multiple channel entries."

INDEX \{ applelmtOrSvc, applelmtorSvcId, applopenChannelIndex \}

$::=\{$ applopenchannelTable 1$\}$

ApplOpenChannelEntry $\quad::=$ SEQUENCE

\{

applElmtOrSvC

applElmtOrSvcId

applopenChannelIndex

applopenChannelopenTime

applopenChannelReadRequests

applOpenChannelReadRequest sLow

applopenChannelReadFailures

applopenChannelBytesRead

applopenchannelBytes ReadLow

applOpenChannellastReadTime

applOpenChannelWriteRequests

applOpenChannelWriteRequests Low

applOpenChannelWriteFailures

applOpenChannelBytesWritten
INTEGER, Unsigned32, Unsigned32, Timestamp, Counter64, Counter32, Counter32, Counter64, Counter32, DateAndTime, Counter64, Counter32, Counter32, Counter64, 


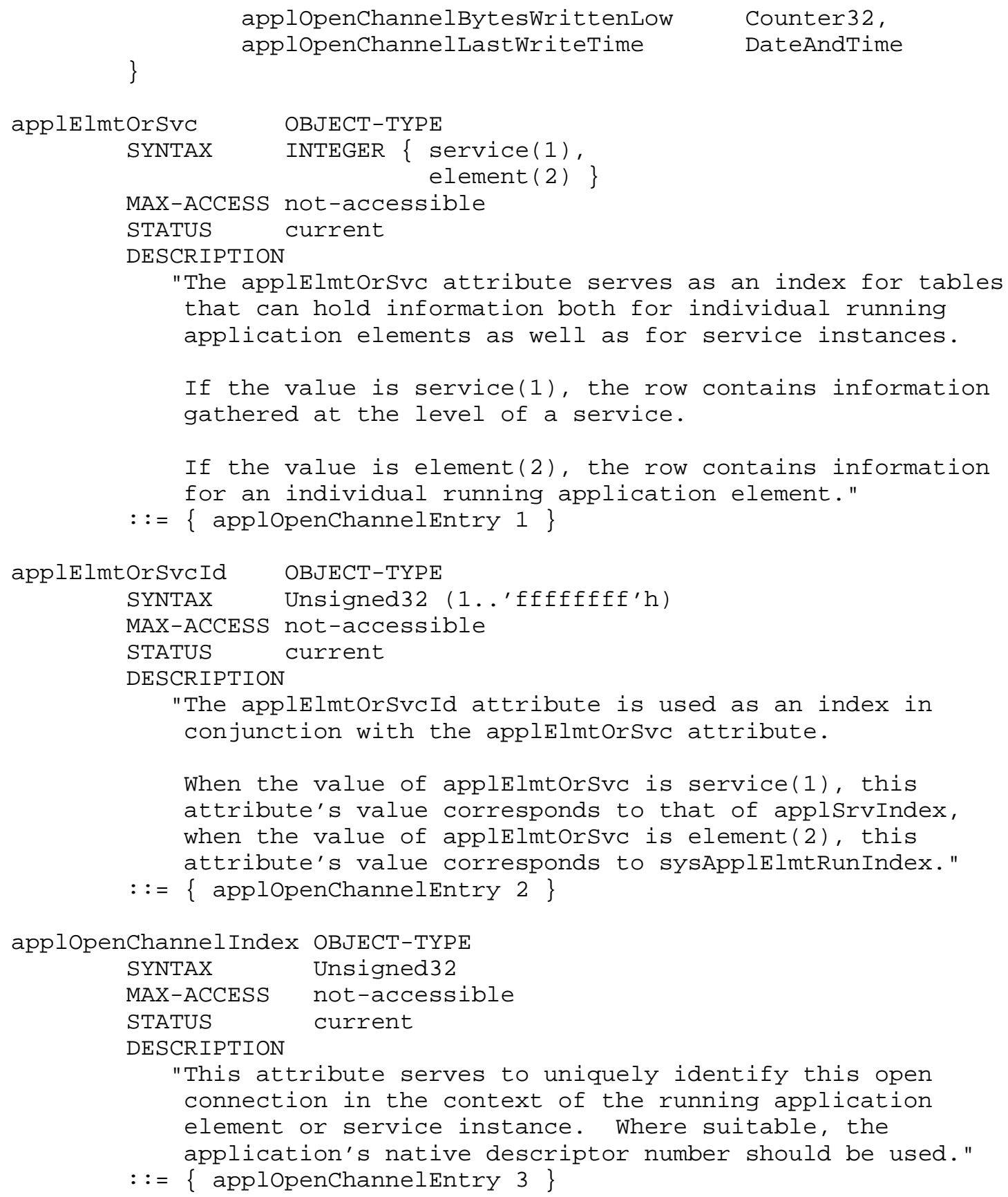




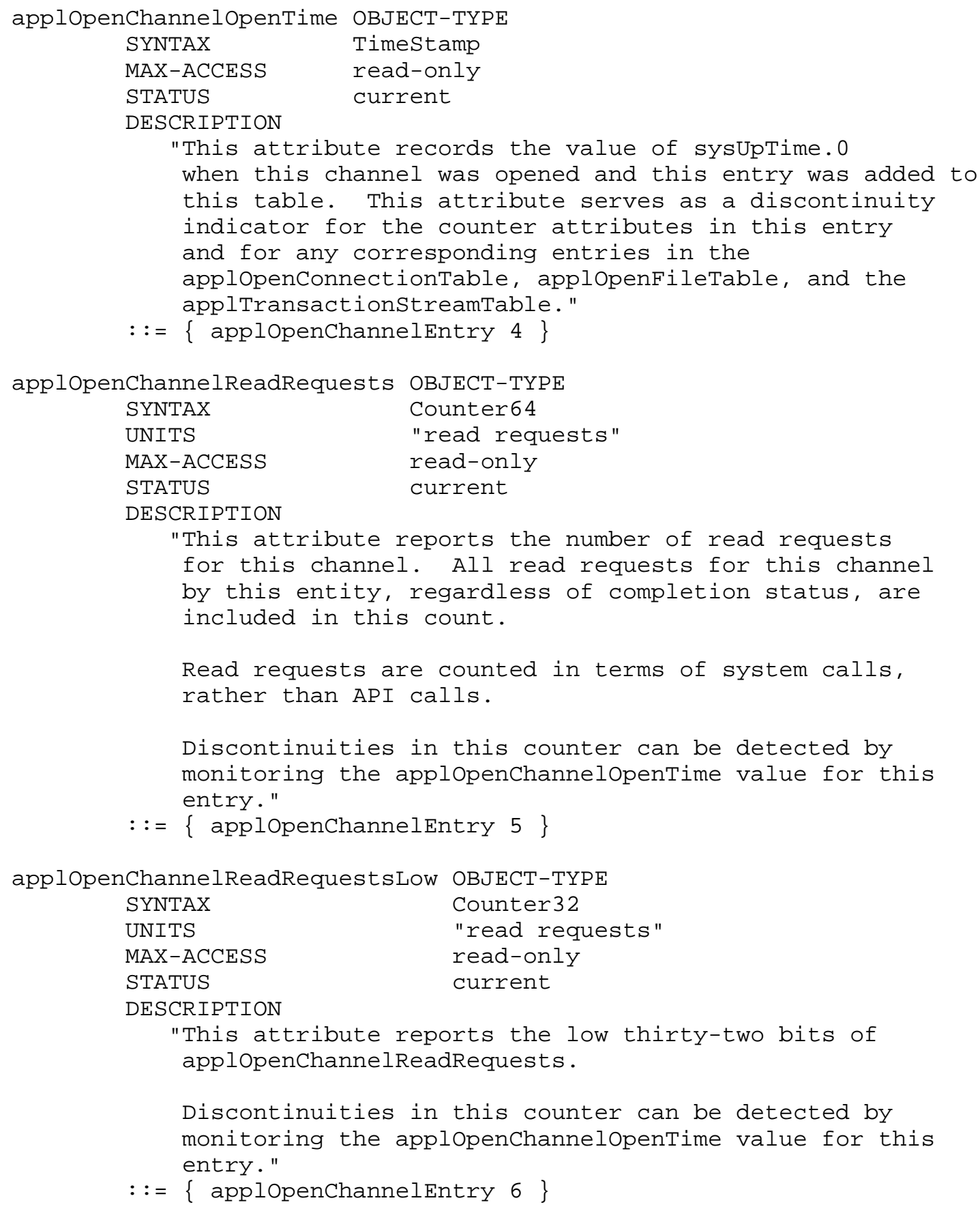




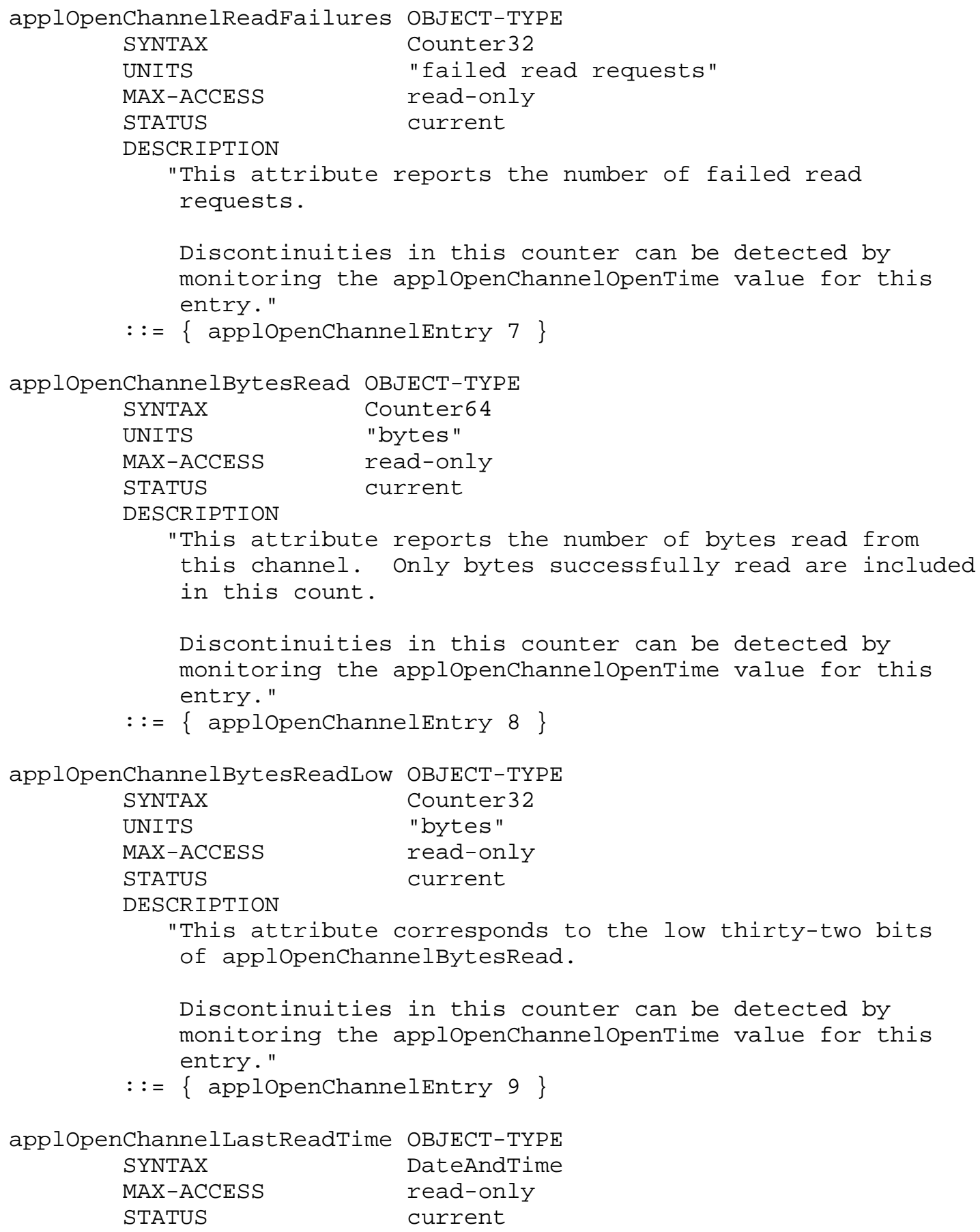

Kalbfleisch, et al. Standards Track [Page 27] 


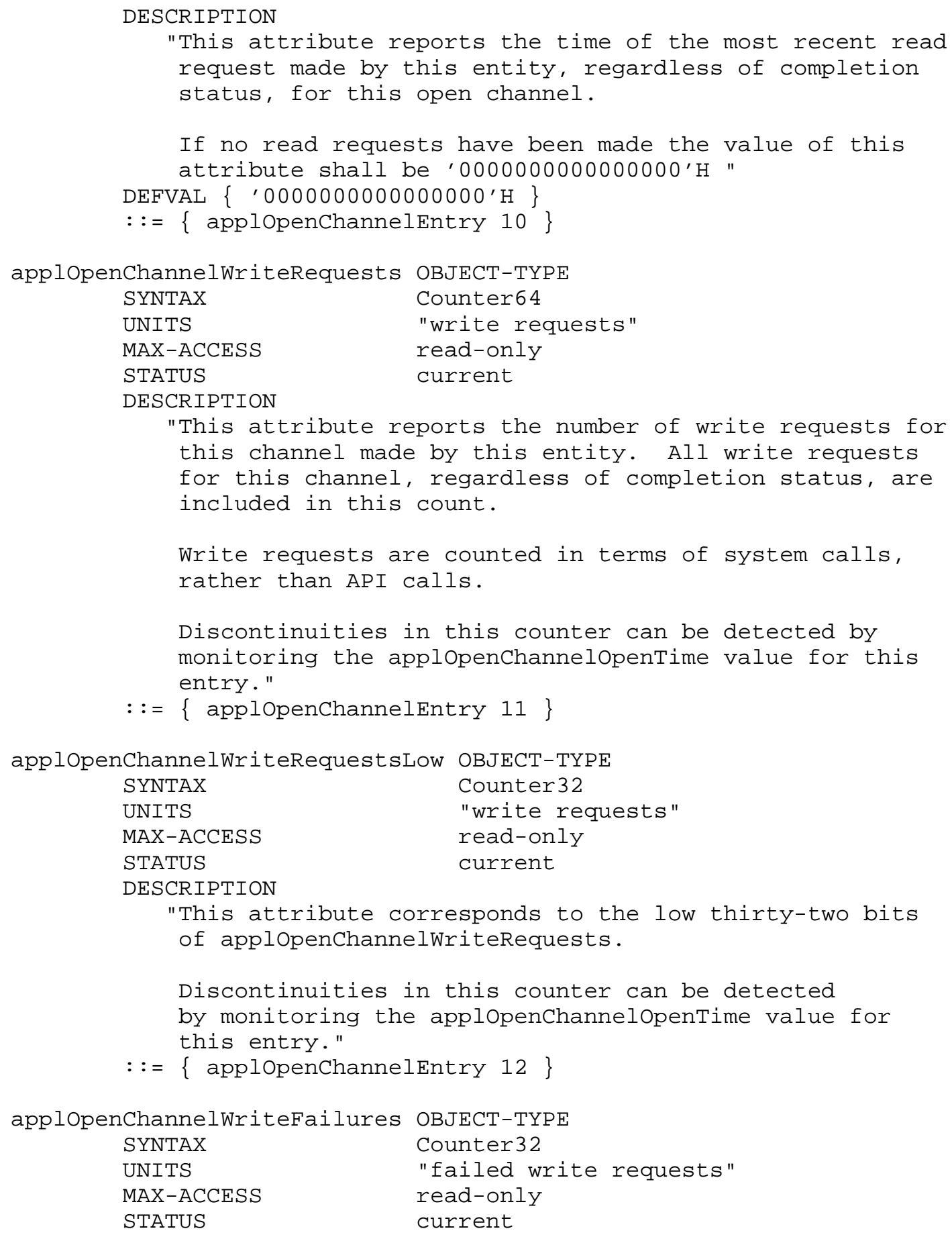

Kalbfleisch, et al. Standards Track [Page 28] 


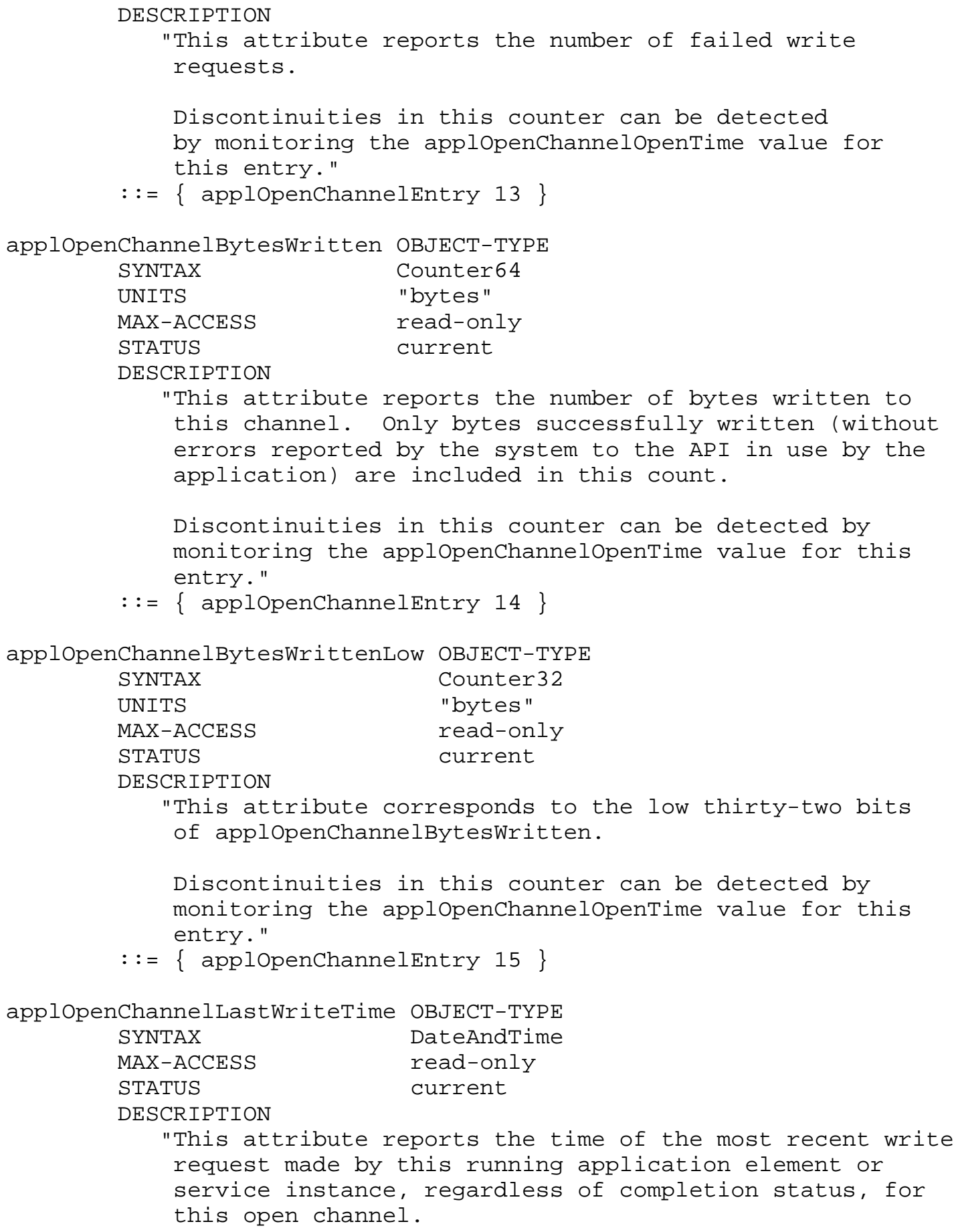




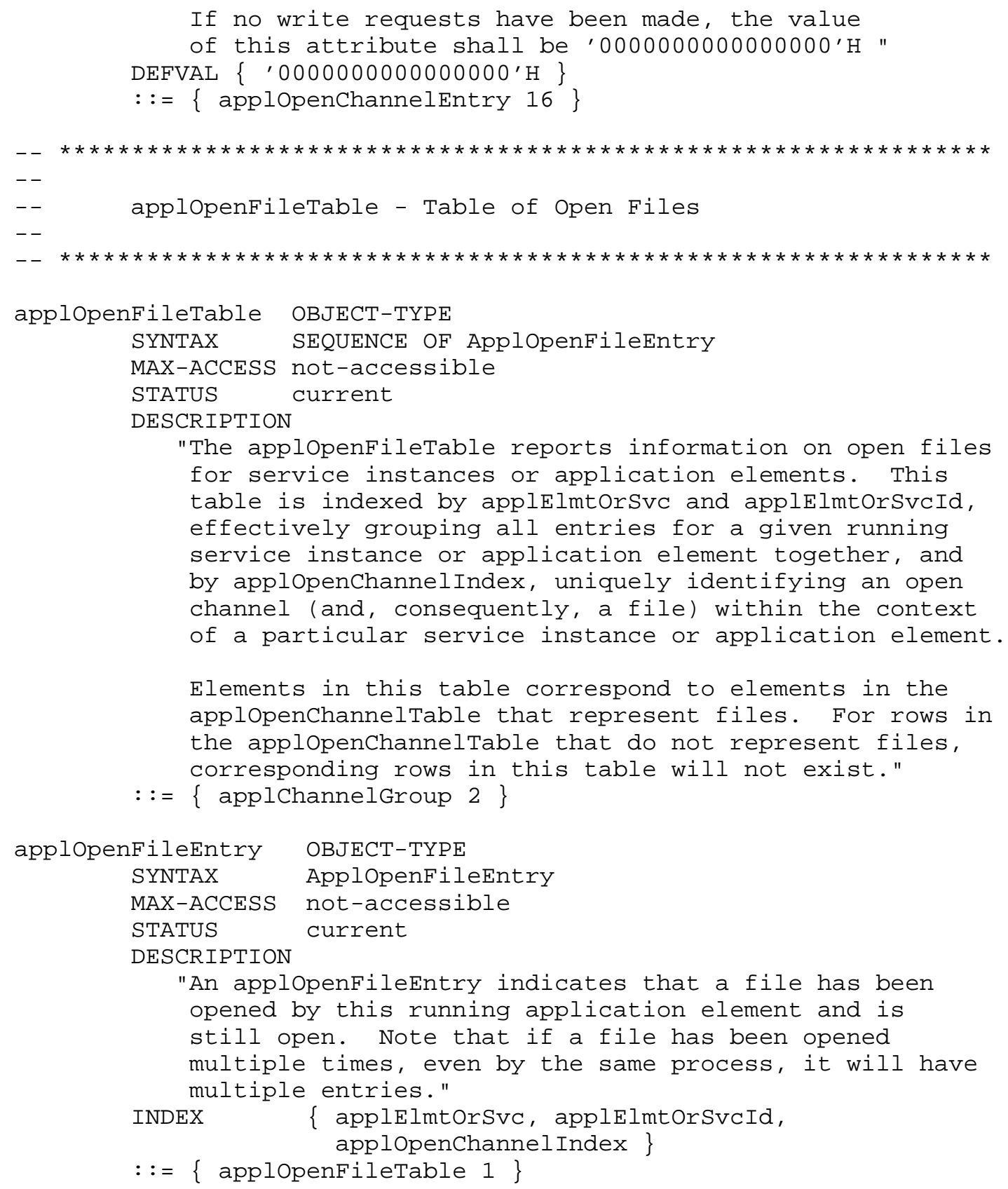




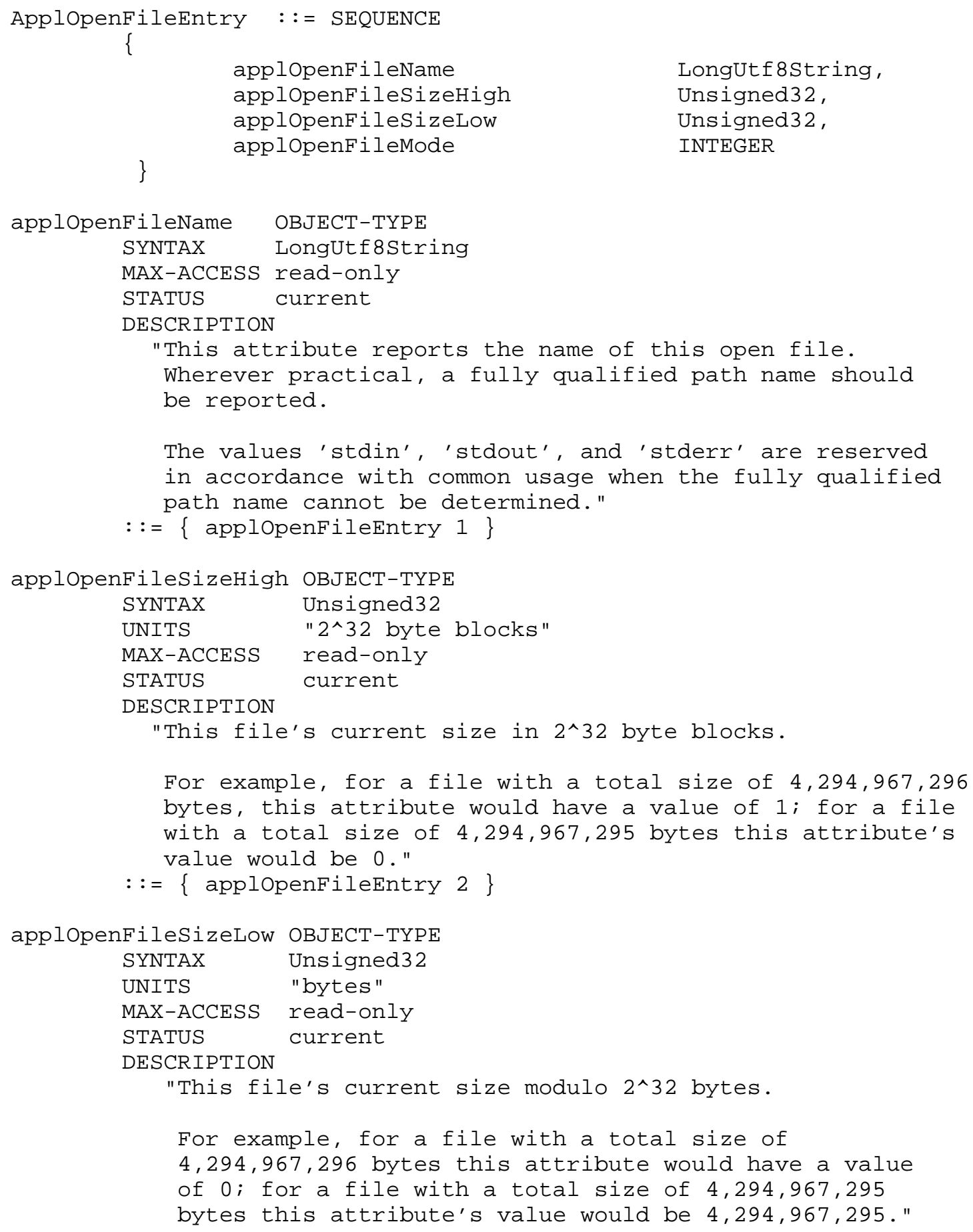




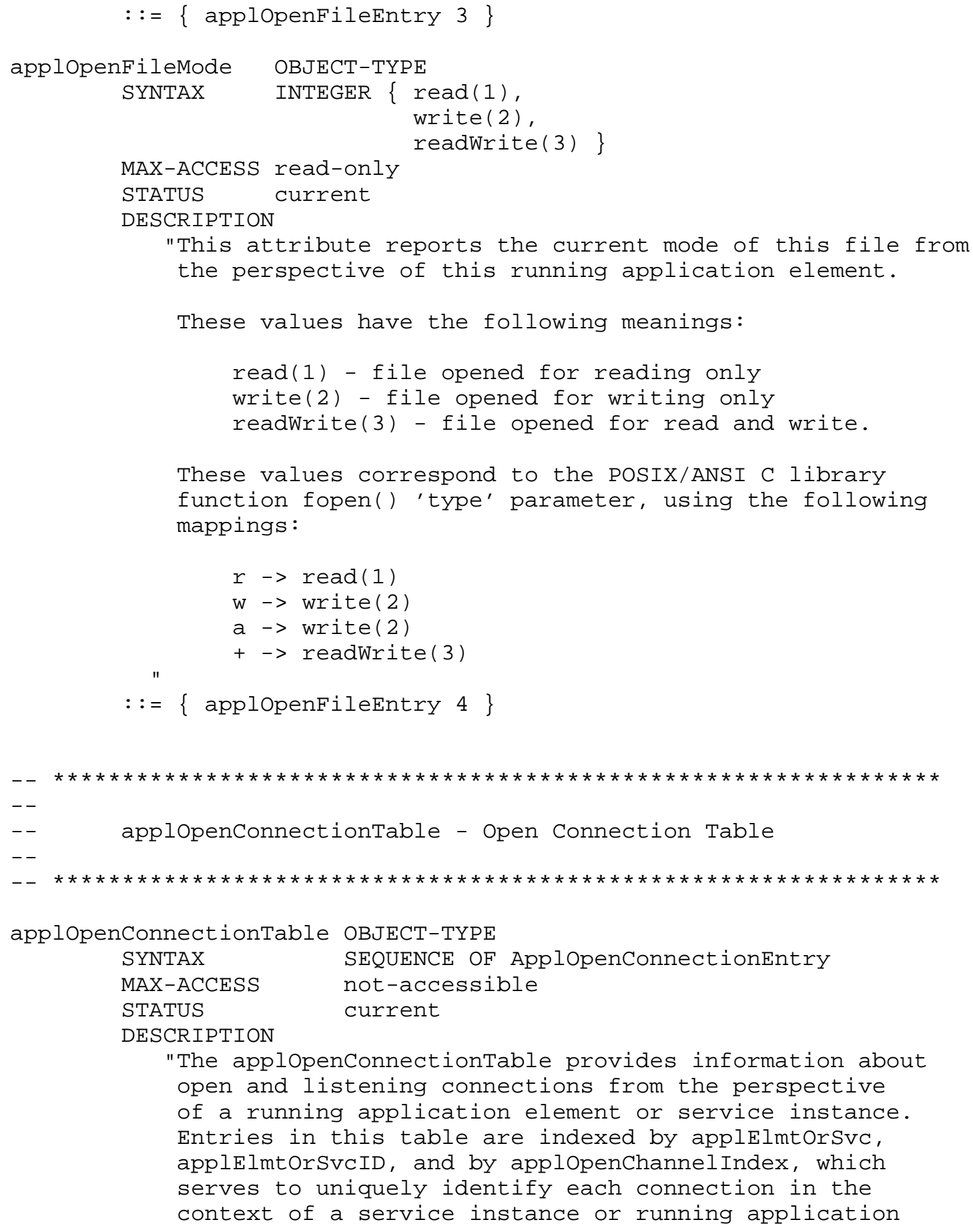




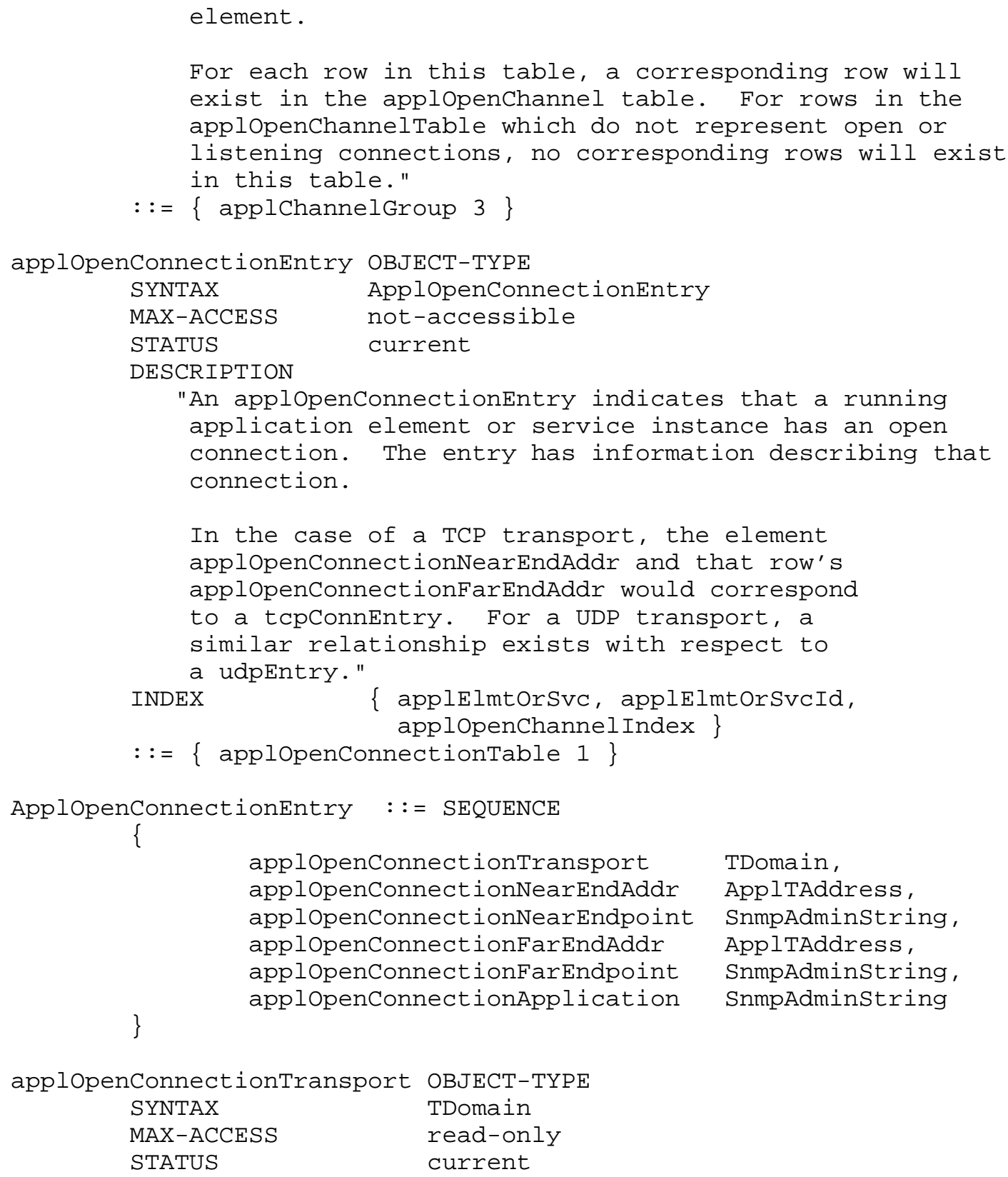




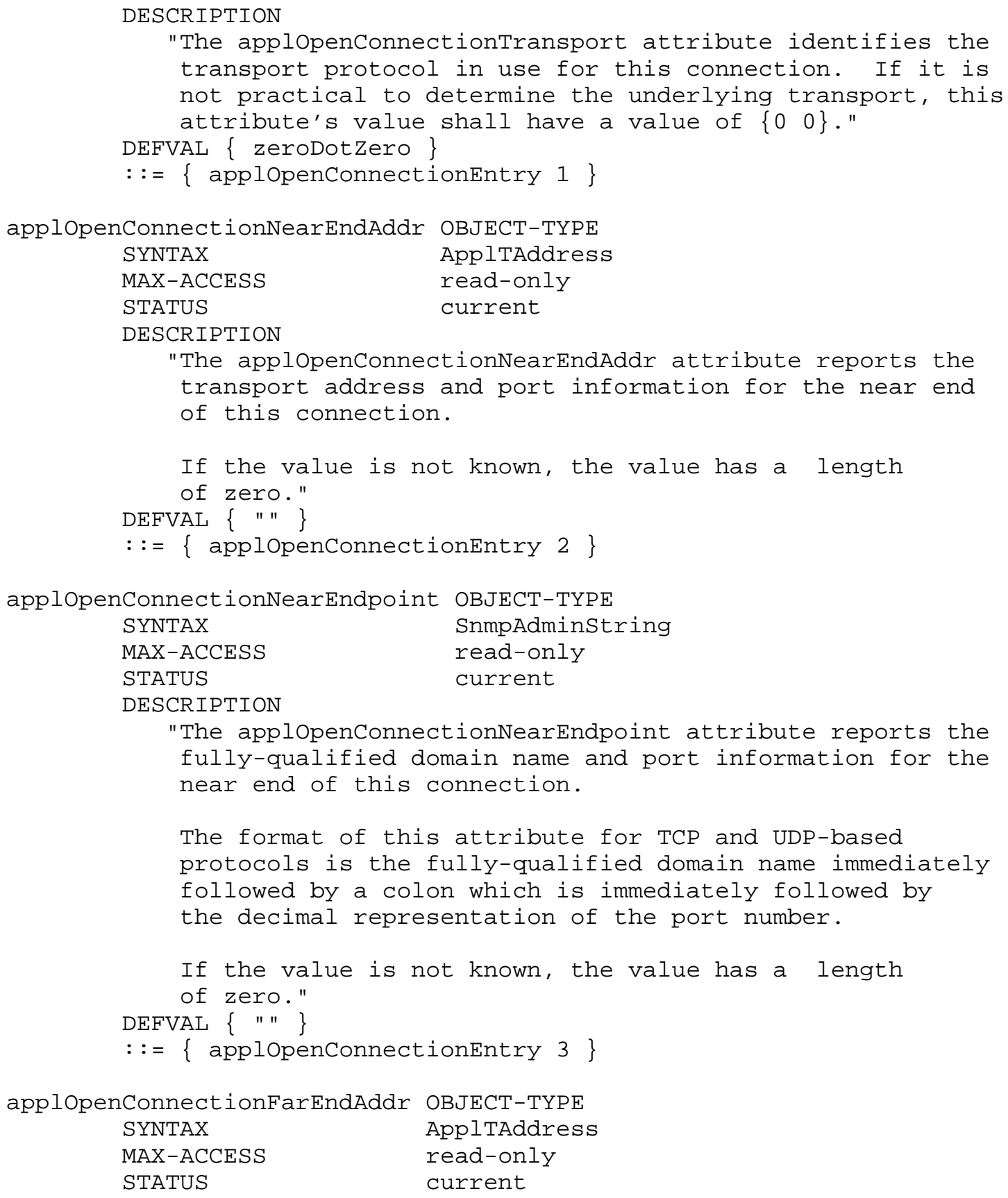

Kalbfleisch, et al. Standards Track [Page 34] 
DESCRIPTION
"The applOpenConnectionFarEndAddr attribute reports the transport address and port information for the far end of this connection.

If not known, as in the case of a connectionless transport, the value of this attribute shall be a zero-length string."

DEFVAL $\{$ " $\}$

$::=\{$ applopenConnectionEntry 4$\}$

applopenConnectionFarEndpoint OBJECT-TYPE

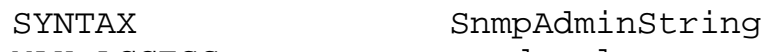




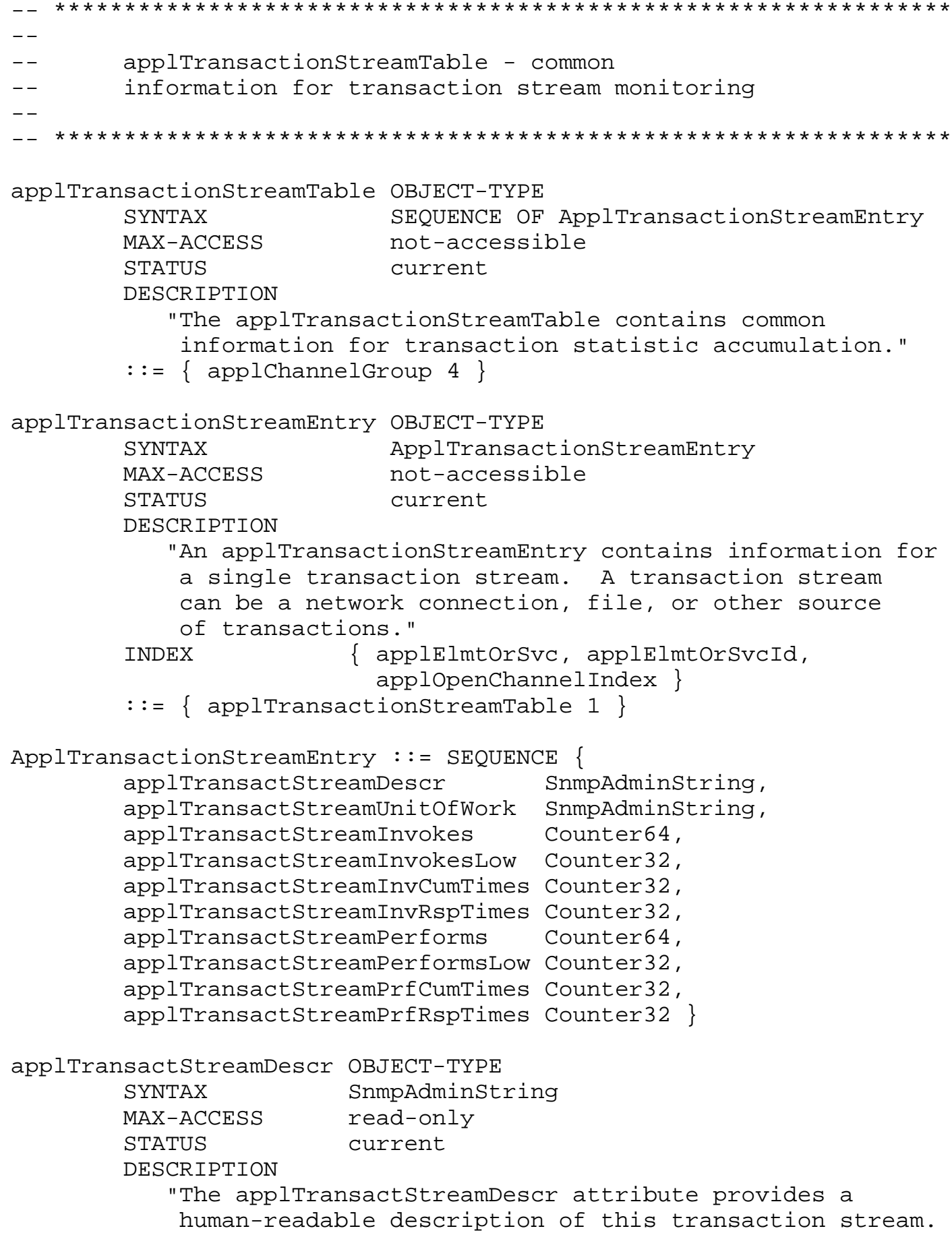

"The applTransactstreamDescr attribute provides a

human-readable description of this transaction stream. 


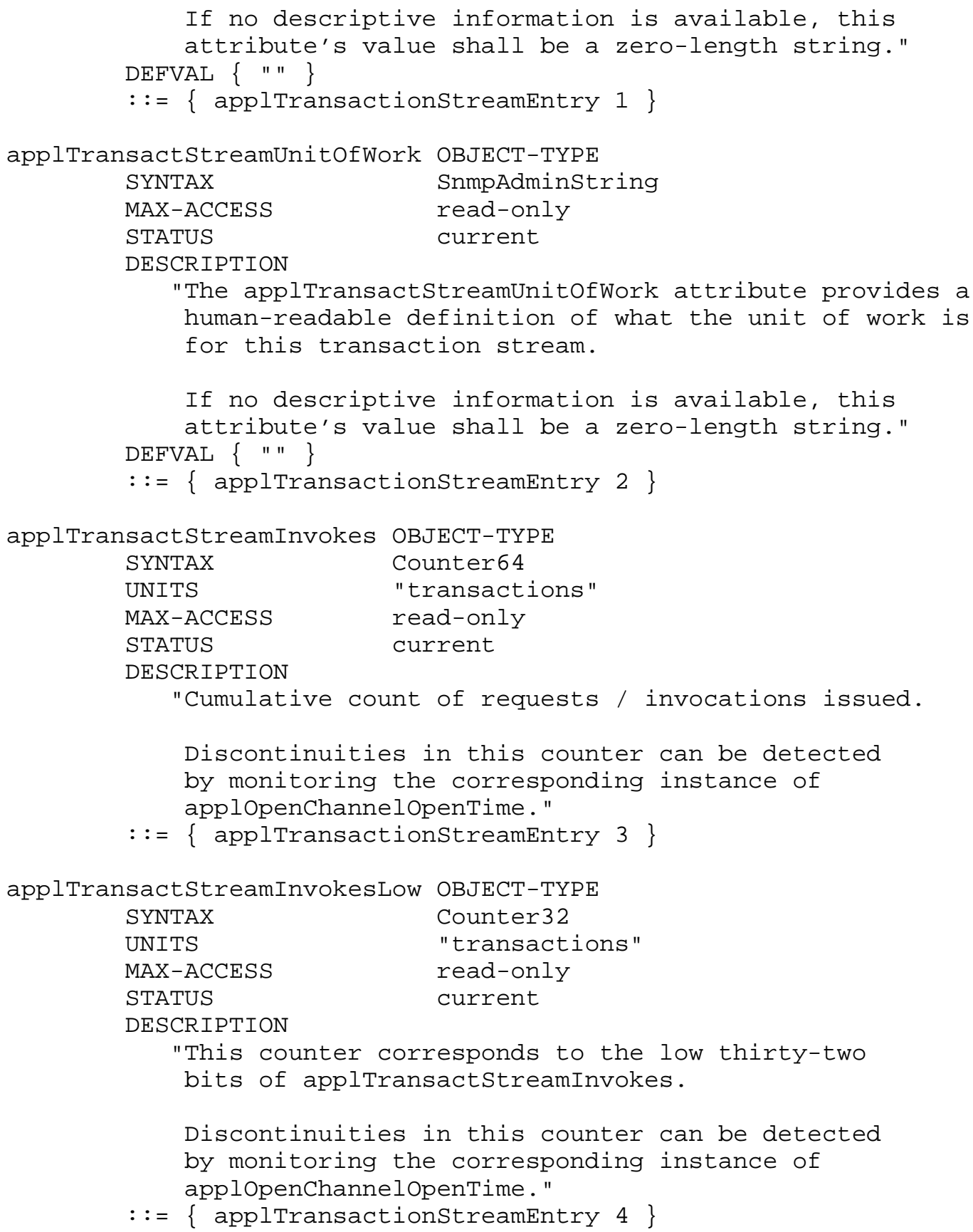

Kalbfleisch, et al.

Standards Track

[Page 37] 


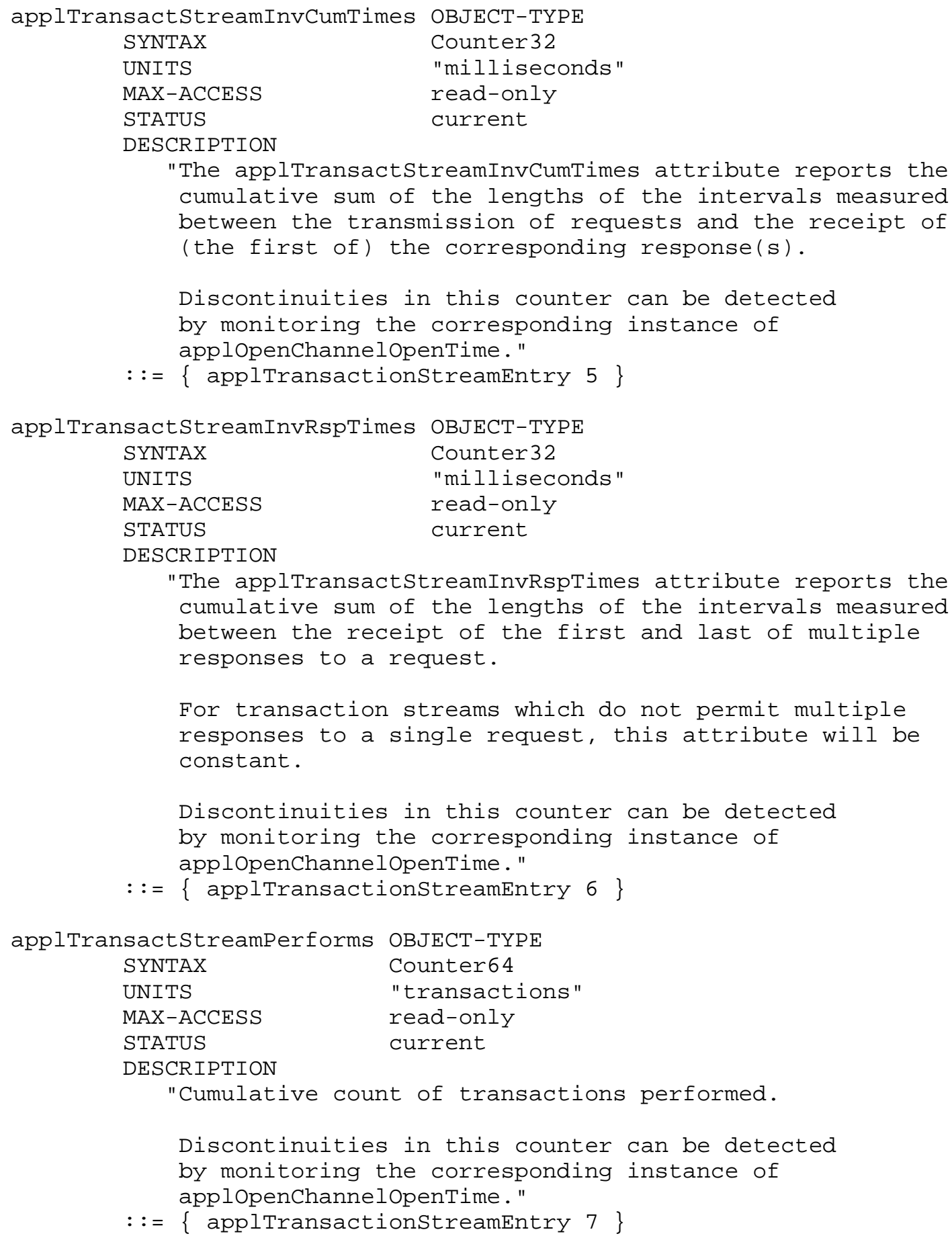

Kalbfleisch, et al.

Standards Track

[Page 38] 


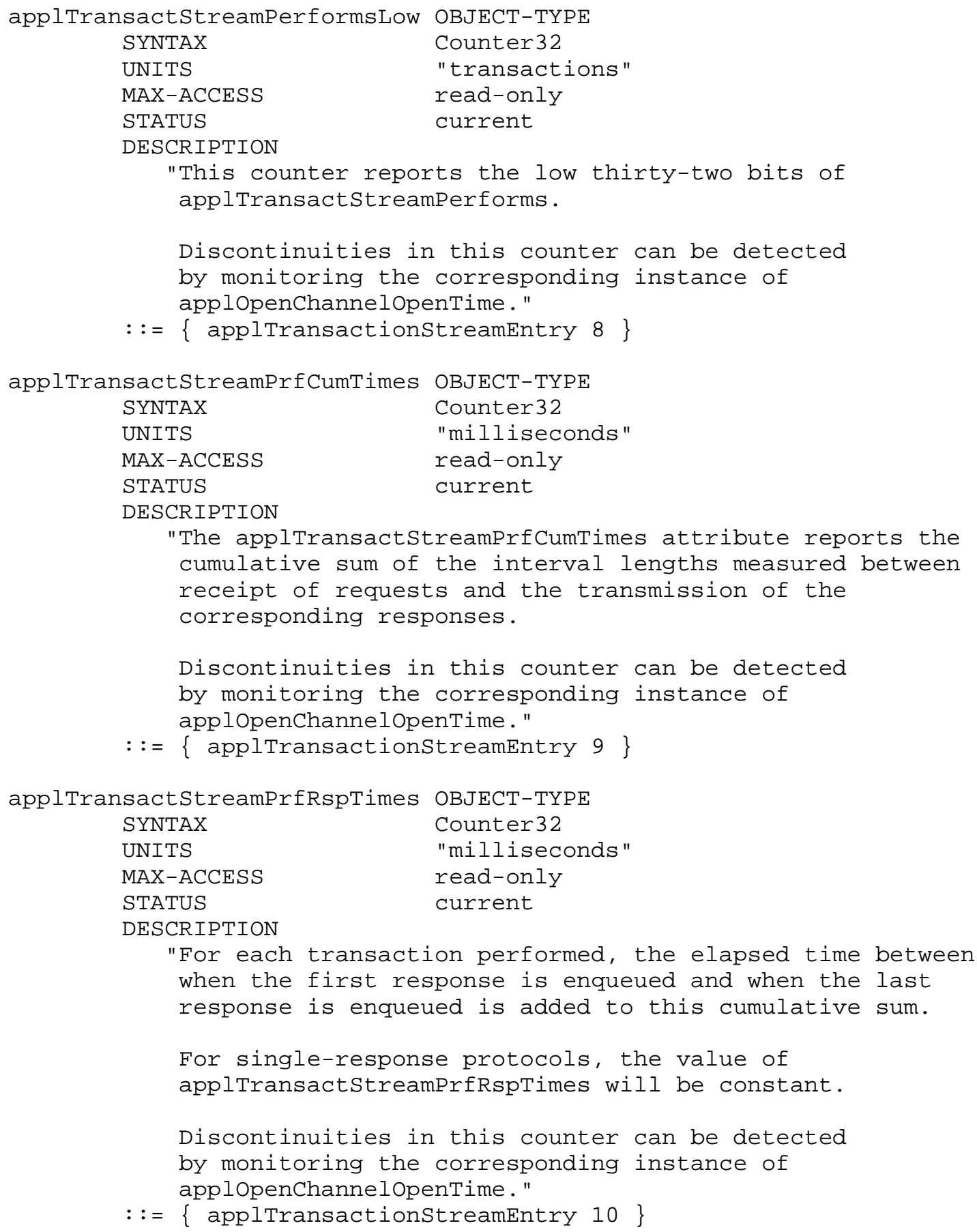

Kalbfleisch, et al.

Standards Track

[Page 39] 


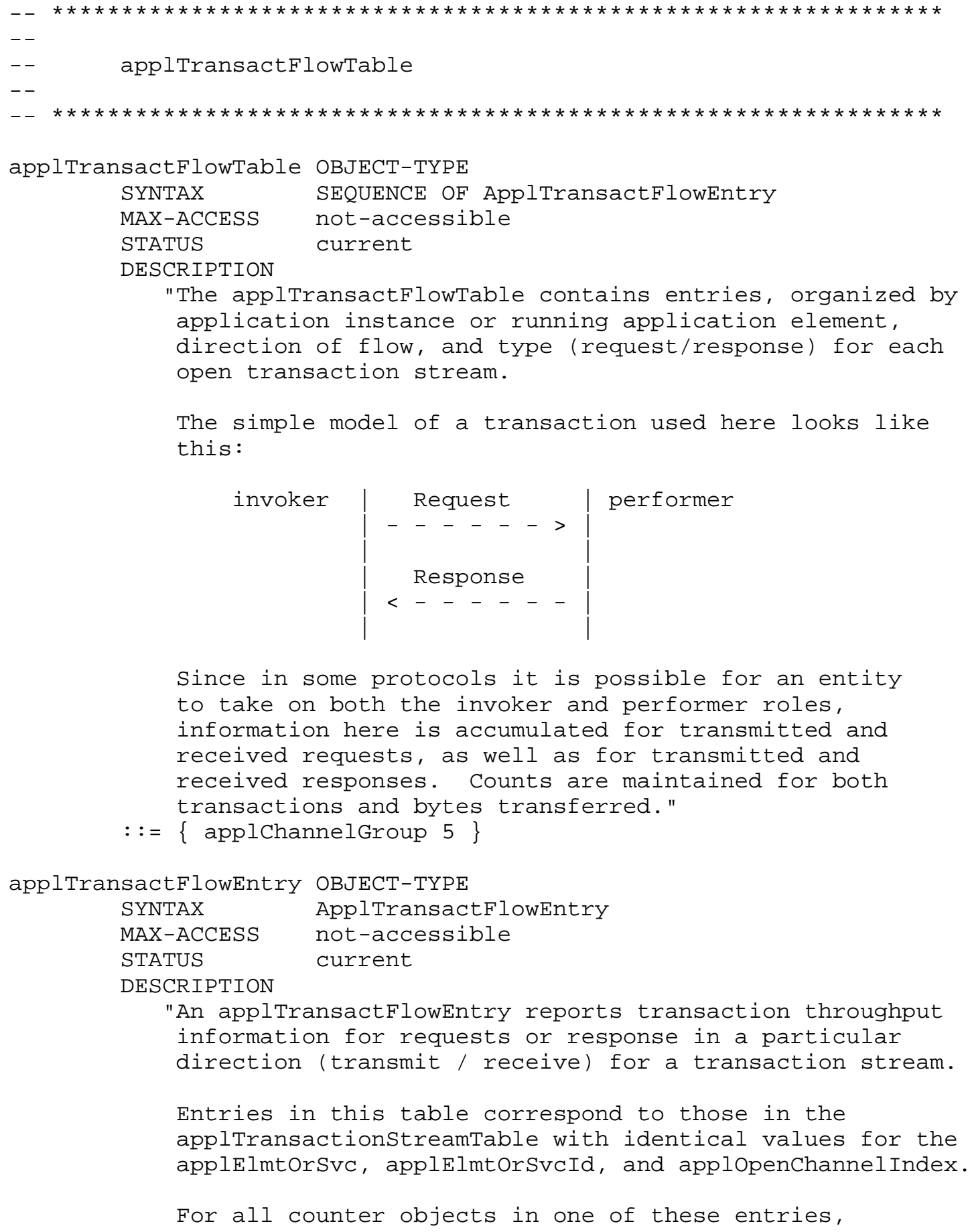




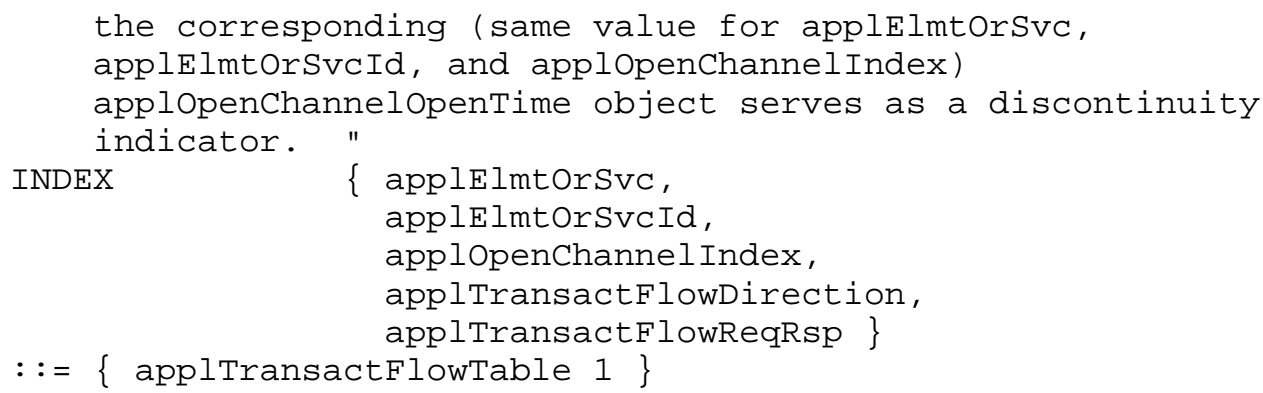




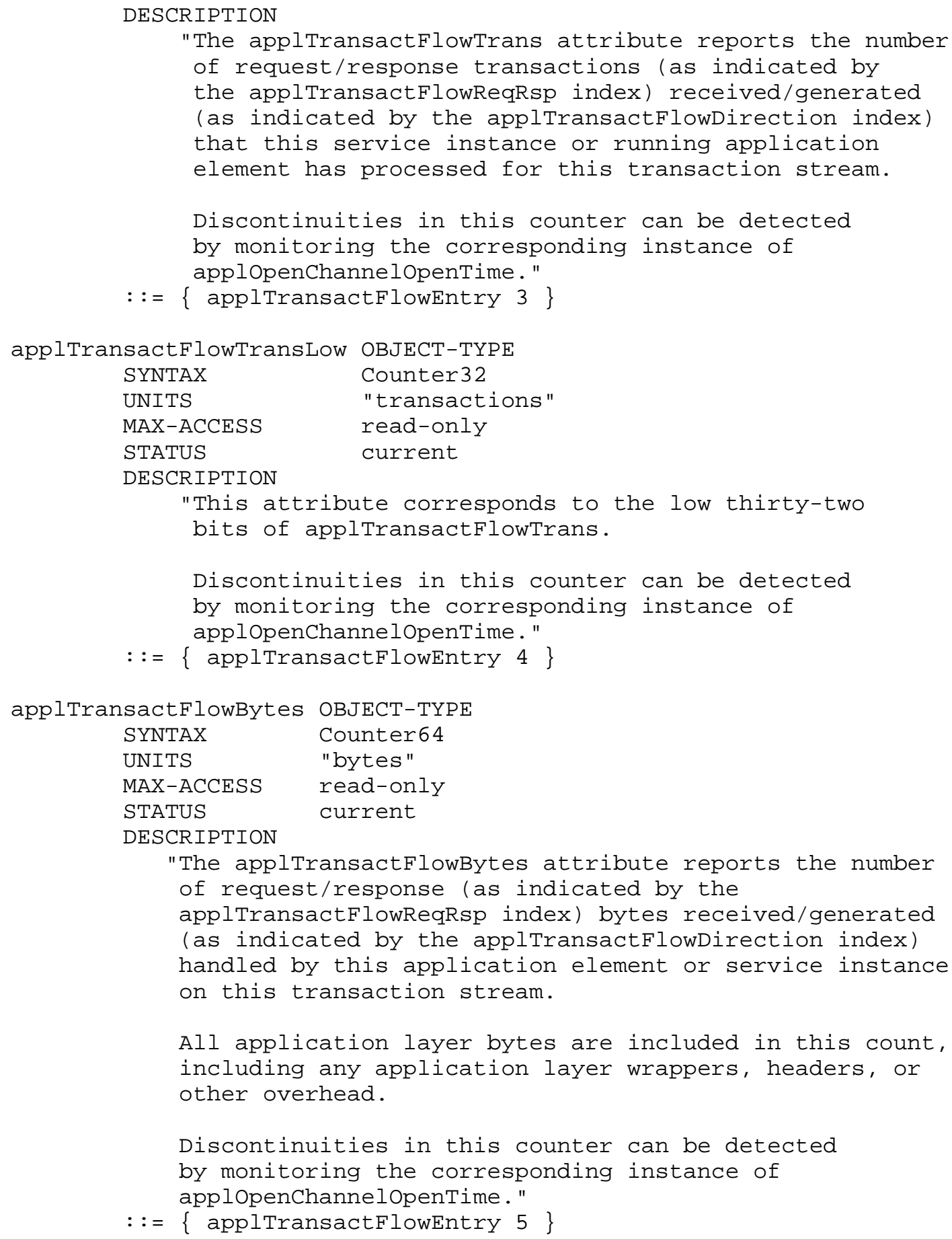

Kalbfleisch, et al.

Standards Track 


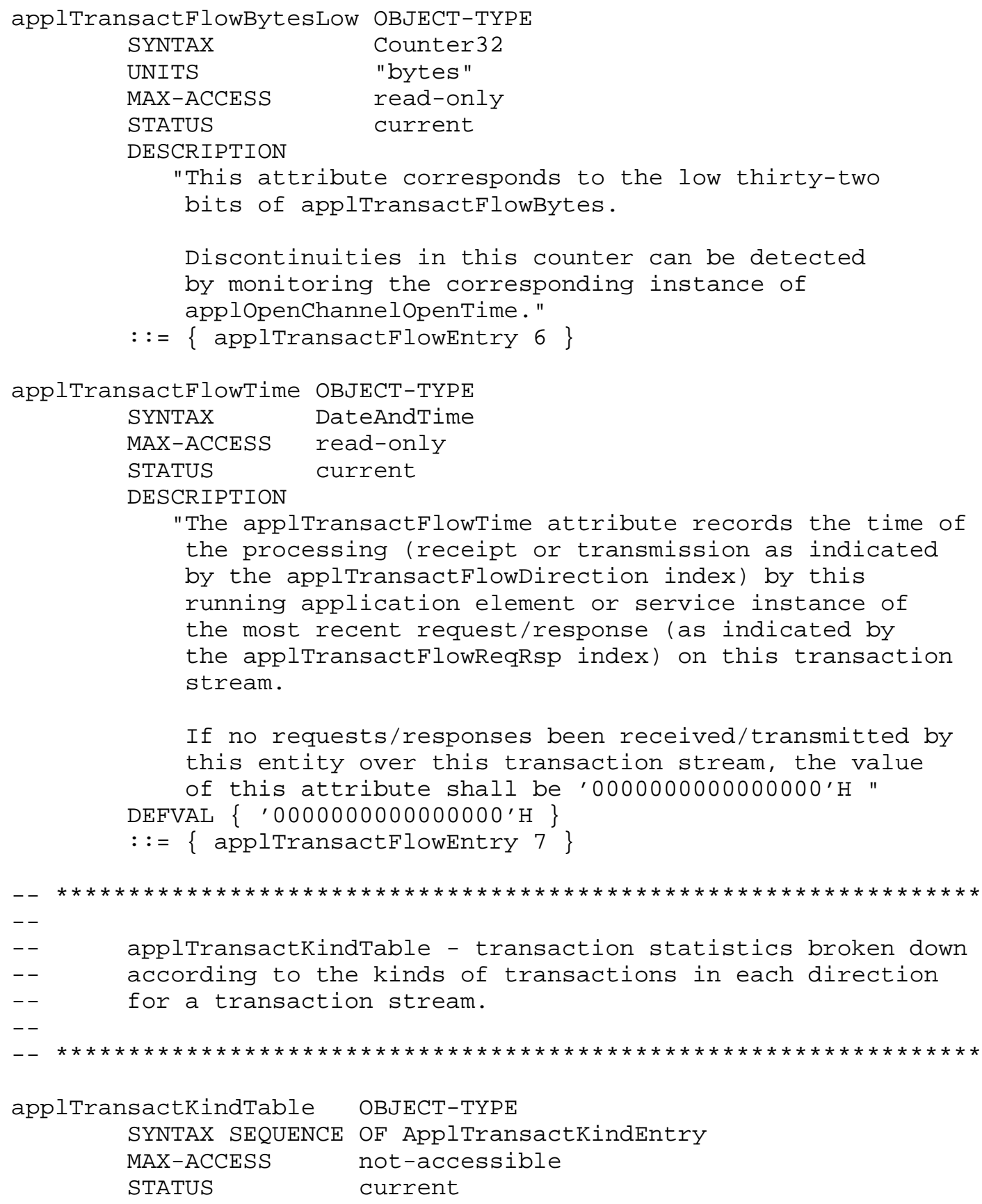




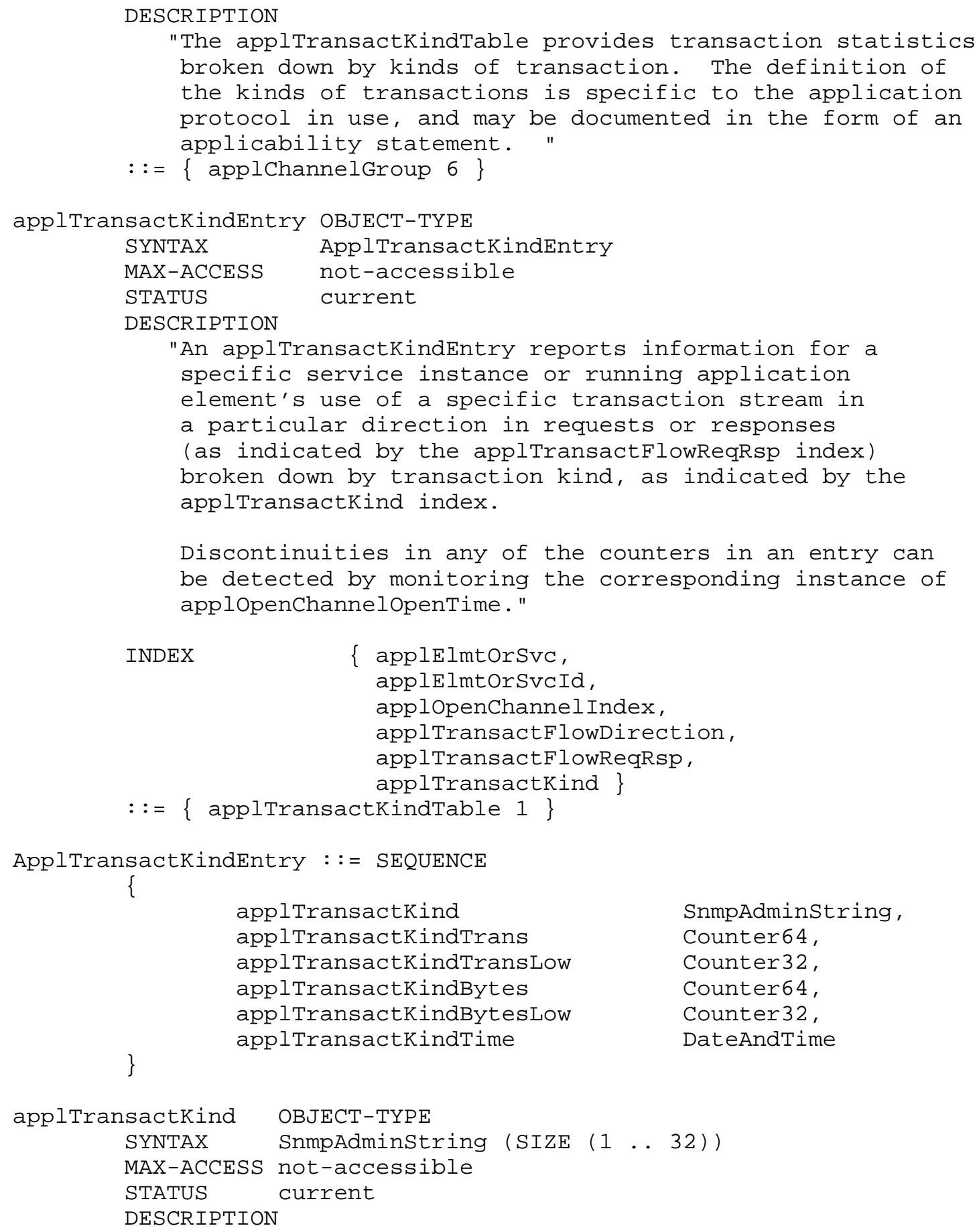




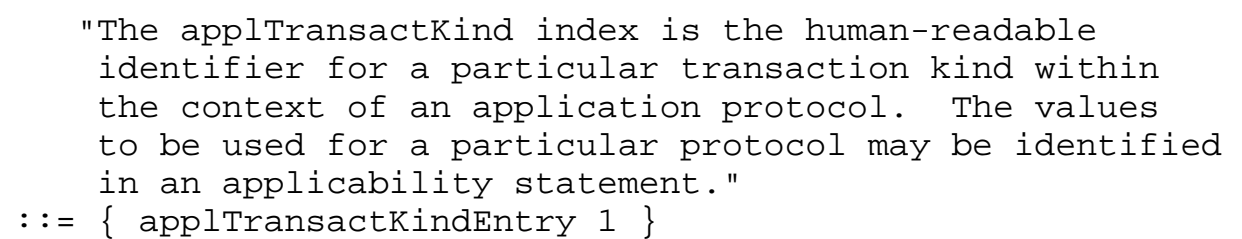

"The applTransactKindTrans attribute reports the number of request/response (as indicated by the applTransactFlowReqRsp index) transactions received/generated (as indicated by the applTransactFlowDirection index) handled by this application instance or application element on this transaction stream for this transaction kind.

Discontinuities in this counter can be detected by monitoring the corresponding instance of applopenChannelopentime."

$::=\{$ applTransactKindEntry 2$\}$

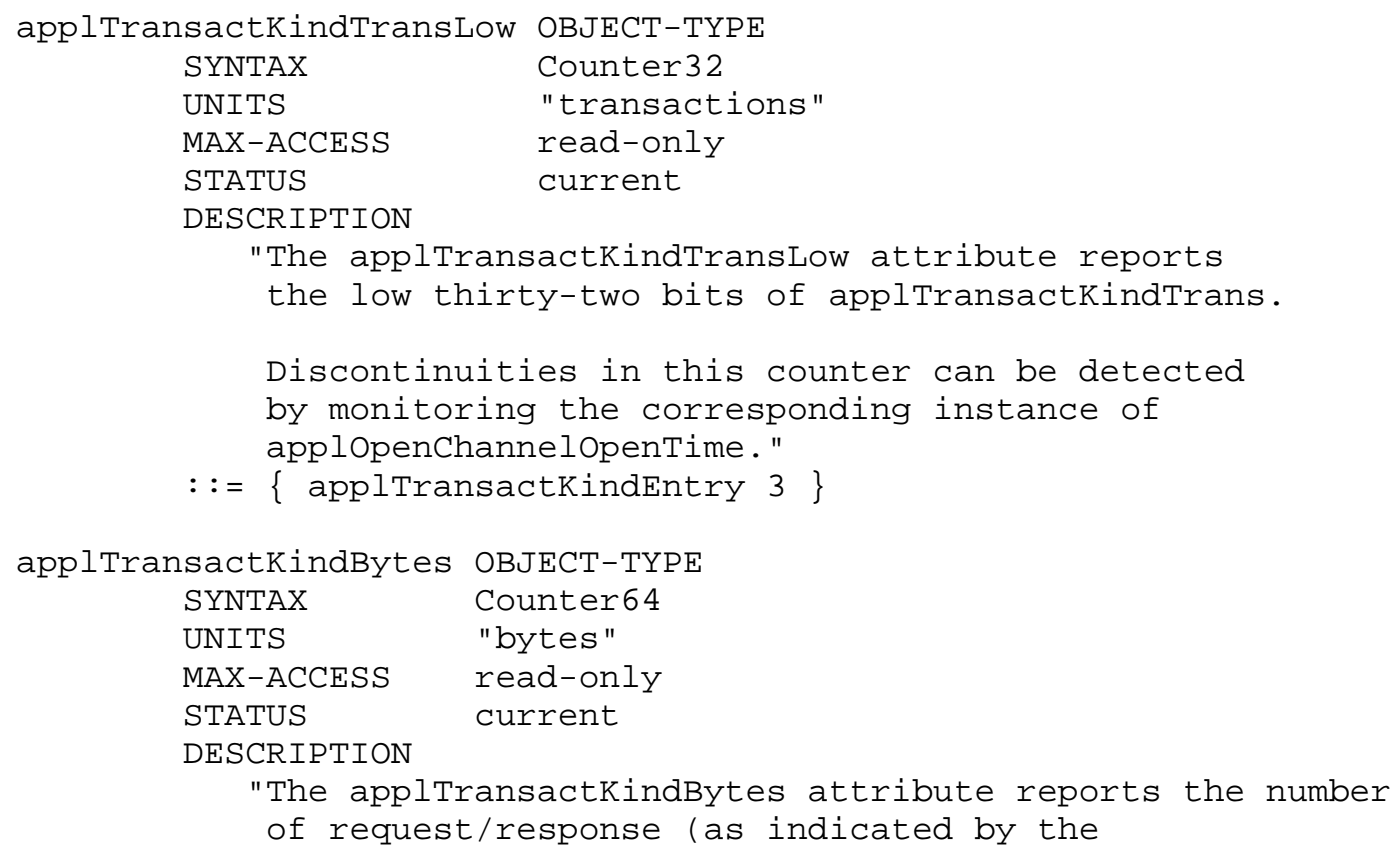


applTransactFlowReqRsp index) bytes received/generated (as indicated by the applTransactFlowDirection index) handled by this application element on this transaction stream for this transaction kind.

All application layer bytes are included in this count, including any application layer wrappers, headers, or other overhead.

Discontinuities in this counter can be detected by monitoring the corresponding instance of applopenChannelopenTime."

$::=\{$ applTransactKindEntry 4$\}$

applTransactKindBytesLow OBJECT-TYPE

SYNTAX Counter32

UNITS "bytes"

MAX-ACCESS read-only

STATUS current

DESCRIPTION

"The applTransactKindBytesLow attribute corresponds

to the low thirty-two bits of appliransactkindBytes.

Discontinuities in this counter can be detected

by monitoring the corresponding instance of applOpenChannelopenTime."

$::=\{$ applTransactKindEntry 5$\}$

applTransactKindTime OBJECT-TYPE

SYNTAX DateAndTime

MAX-ACCESS read-only

STATUS current

DESCRIPTION

"The applTransactKindTime attribute records the time of

the processing (receipt or transmission as indicated by the applTransactFlowDirection index) by this running application element or service instance of the most recent request/response (as indicated by the applTransactFlowReqRsp index) of this kind of transaction on this transaction stream.

If no requests/responses of this kind been received/transmitted by this running application element or service instance over this transaction stream, the value of this attribute shall be '0000000000000000'H " DEFVAL $\quad$ ' $0000000000000000 ' \mathrm{H}\}$ $::=\{$ applTransactKindEntry 6$\}$ 


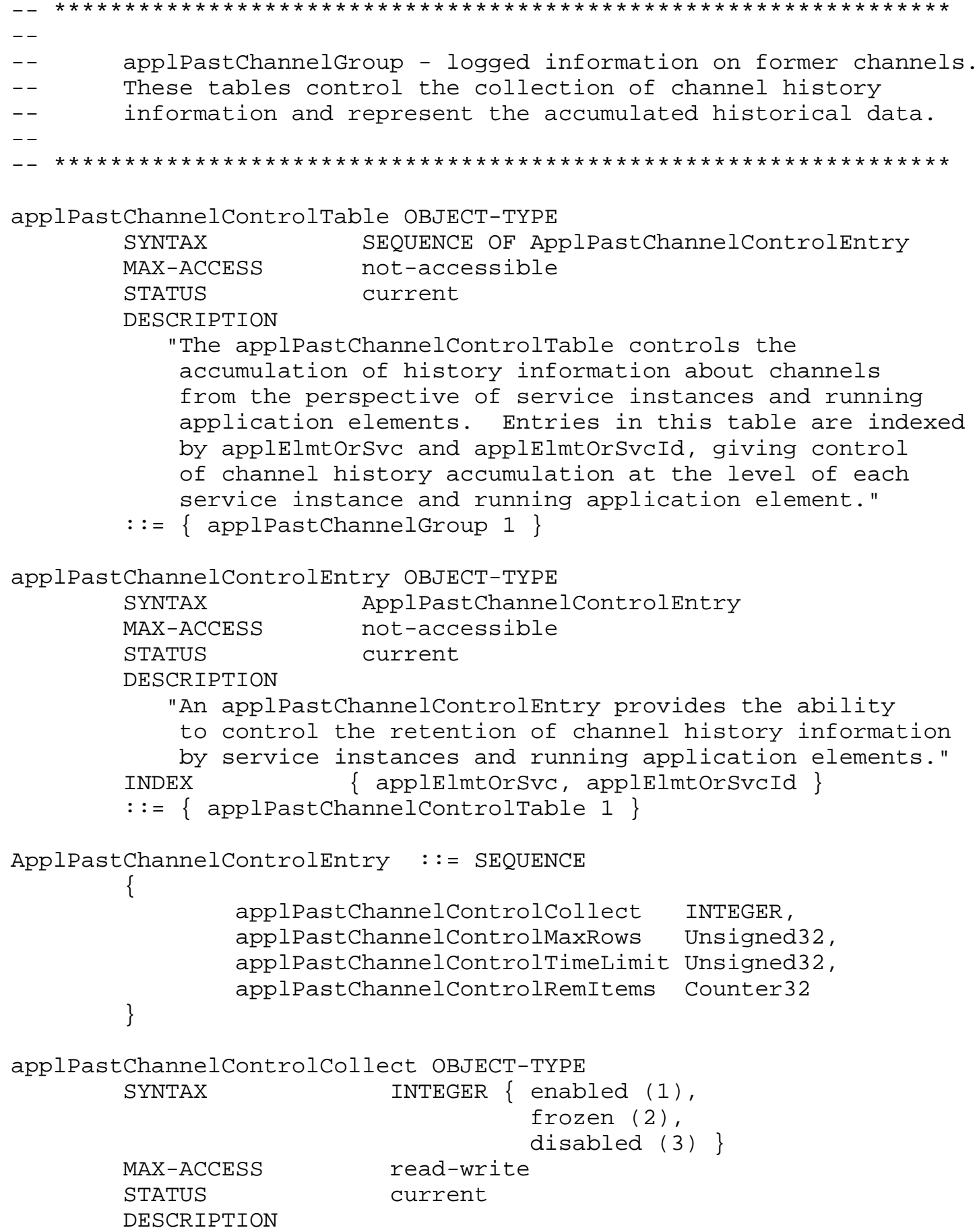


"When the value of applpastChannelcontrolcollect is

'enabled', each time the corresponding running application element or service instance closes an open channel a new entry will be added to the applpastchannelTable.

When the value of applpastChannelcontrolcollect is 'frozen', no new entries are added to the applpastChannelTable for this running application element or service instance, and old entries are not aged out.

When the value of applpastChannelcontrolcollect is 'disabled', all entries are removed from applpastChannelTable for this running application or service instance, and no new entries are added." DEFVAL \{ enabled \} $::=\{$ applpastChannelControlentry 1$\}$

applPastChannelControlMaxRows OBJECT-TYPE

SYNTAX Unsigned32

UNITS "channel history entries"

MAX-ACCESS read-write

STATUS current

DESCRIPTION

"The maximum number of entries allowed in the applpastChannelTable for this running application element or service instance. Once the number of rows for this running application element or service instance in the applPastChannelTable reaches this value, when new entries are to be added the management subsystem will make room for them by removing the oldest entries. Entries will be removed on the basis of oldest applPastChannelcloseTime value first."

DEFVAL $\quad\{500\}$

$::=\{$ applpastChannelControlEntry 2$\}$

applPastChannelControlTimeLimit OBJECT-TYPE

SYNTAX Unsigned32

UNITS "seconds"

MAX-ACCESS read-write

STATUS current

DESCRIPTION

"The maximum time in seconds which an entry for this running application element or service instance may exist in the applpastChannelTable before it is removed. Any entry that is older than this value will be removed (aged out) from the table, unless the 


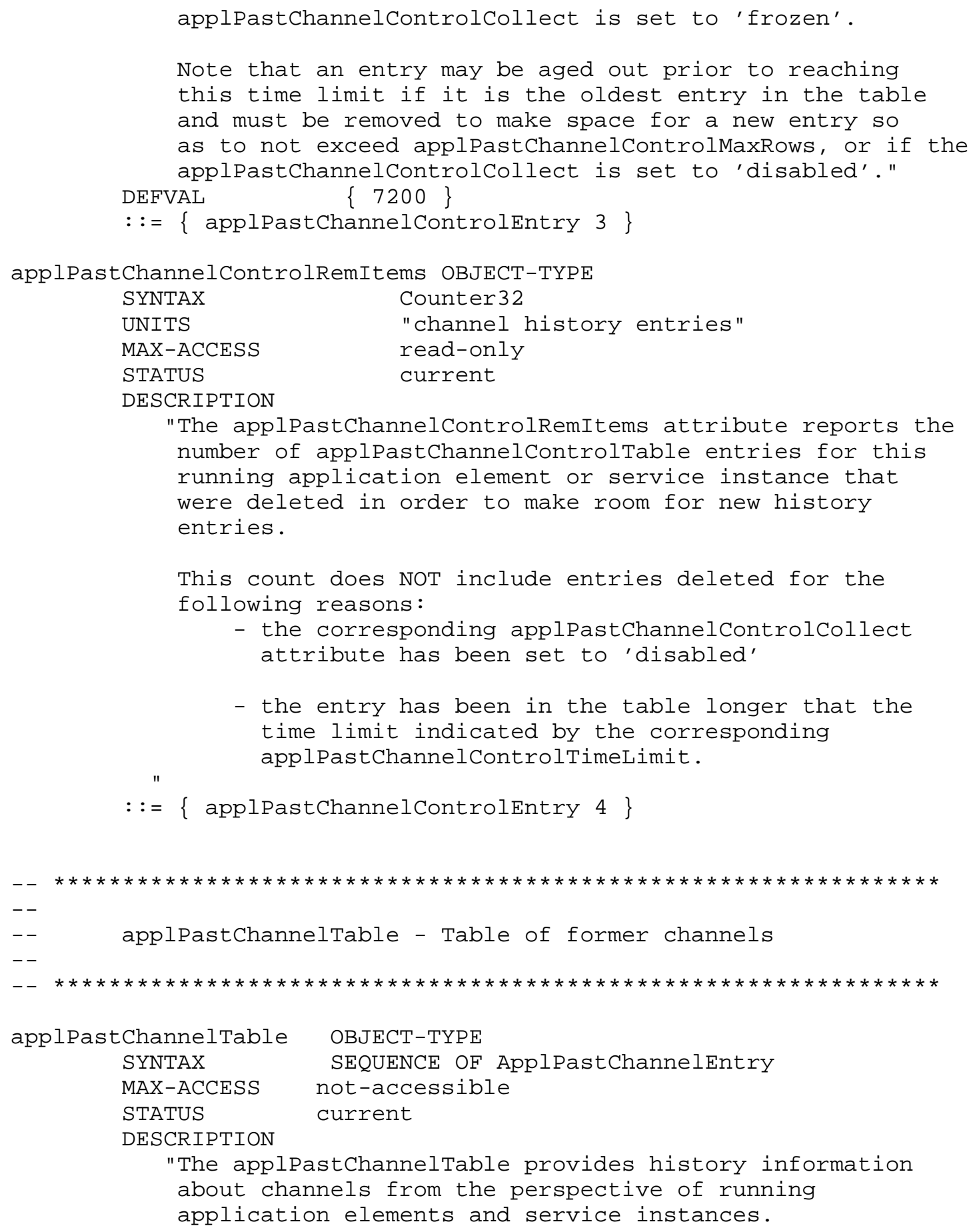




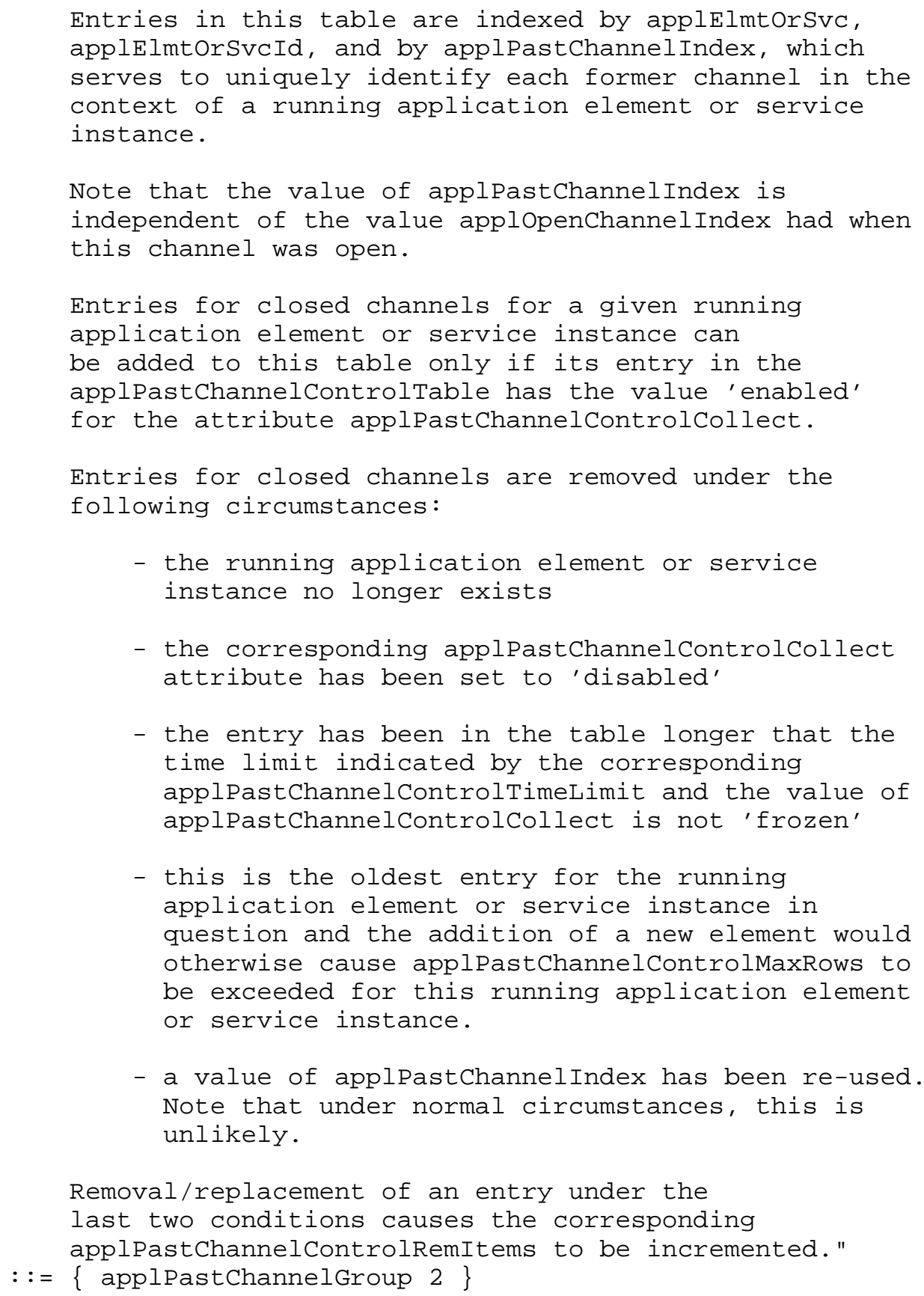

Kalbfleisch, et al.

Standards Track

[Page 50] 


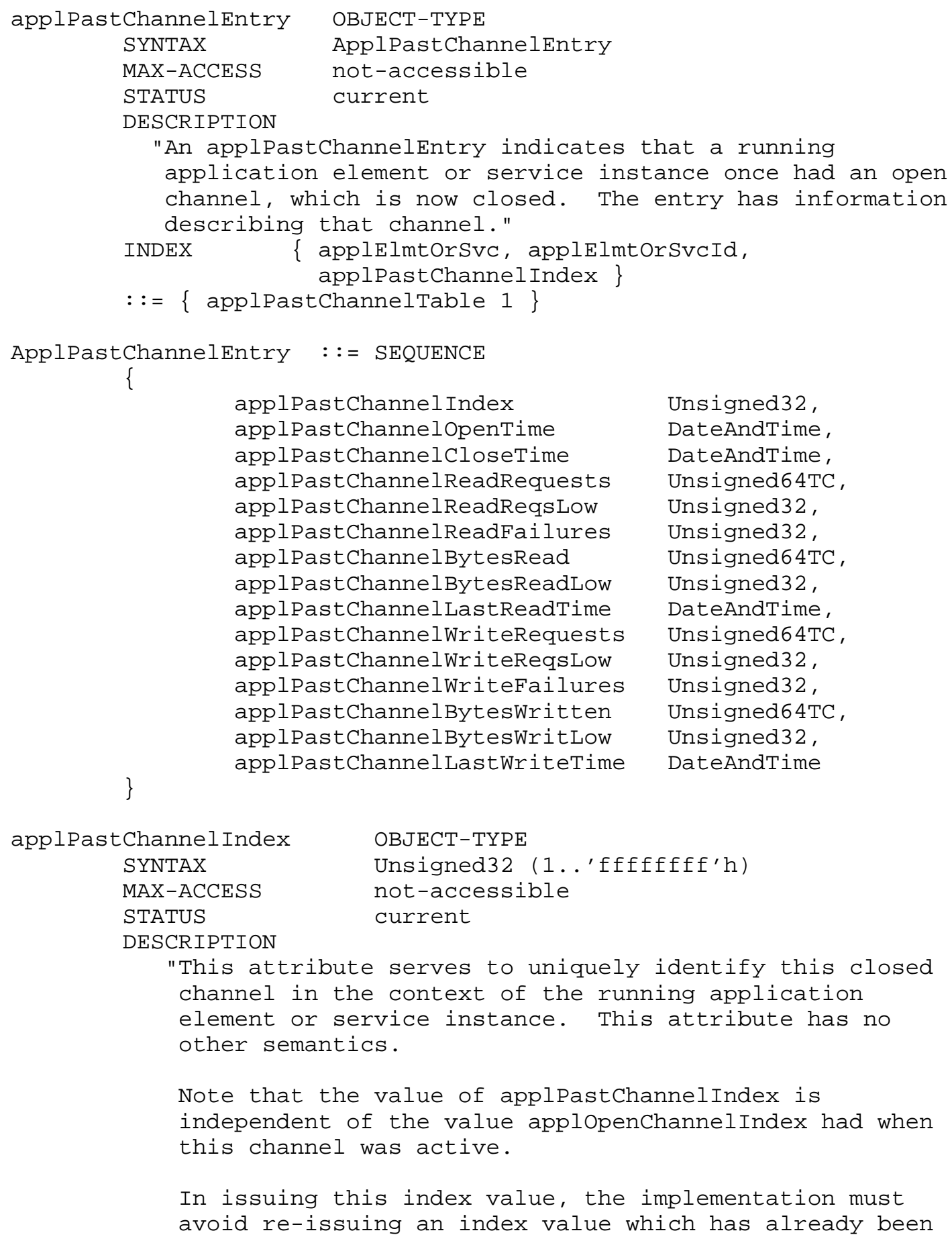




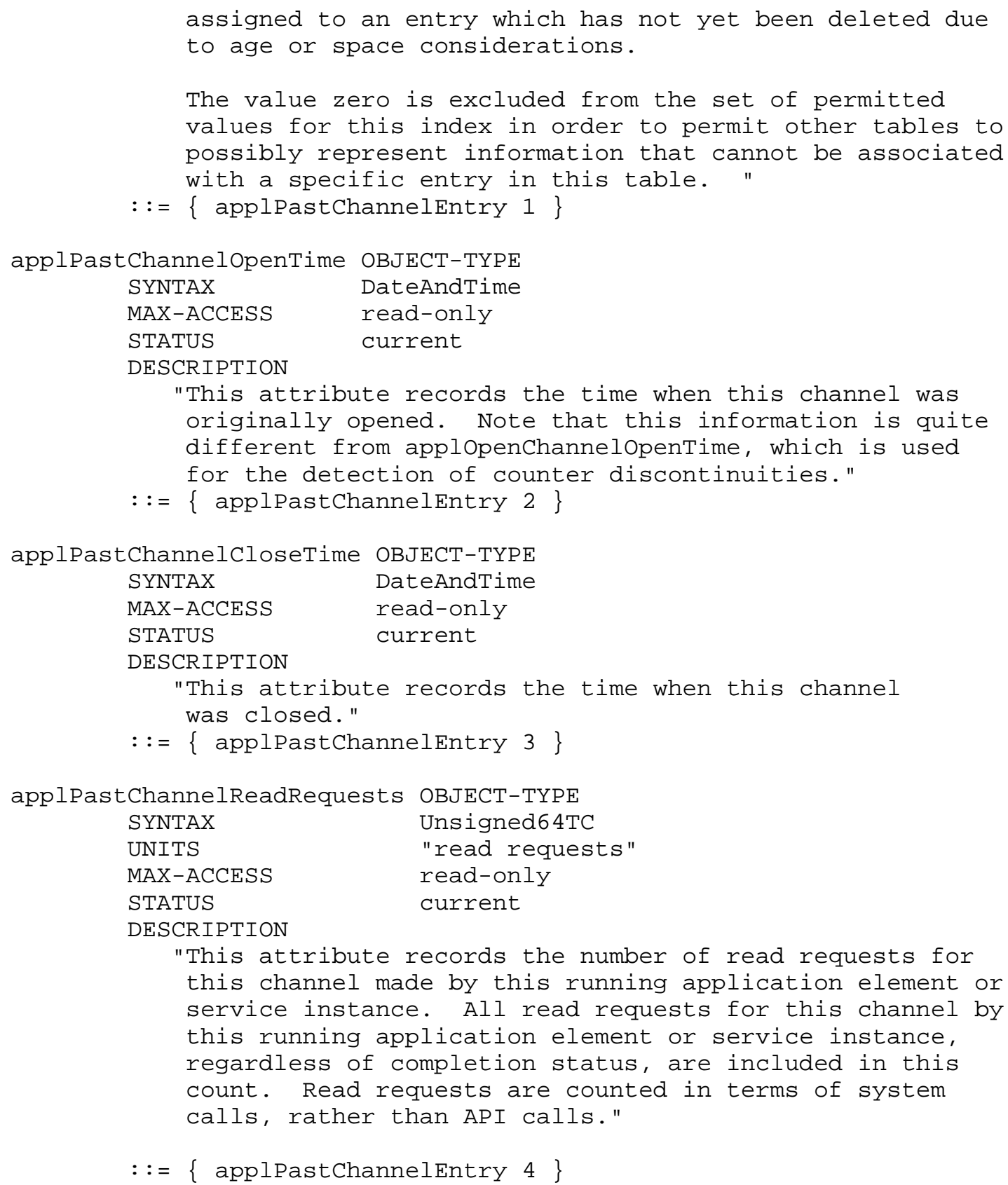




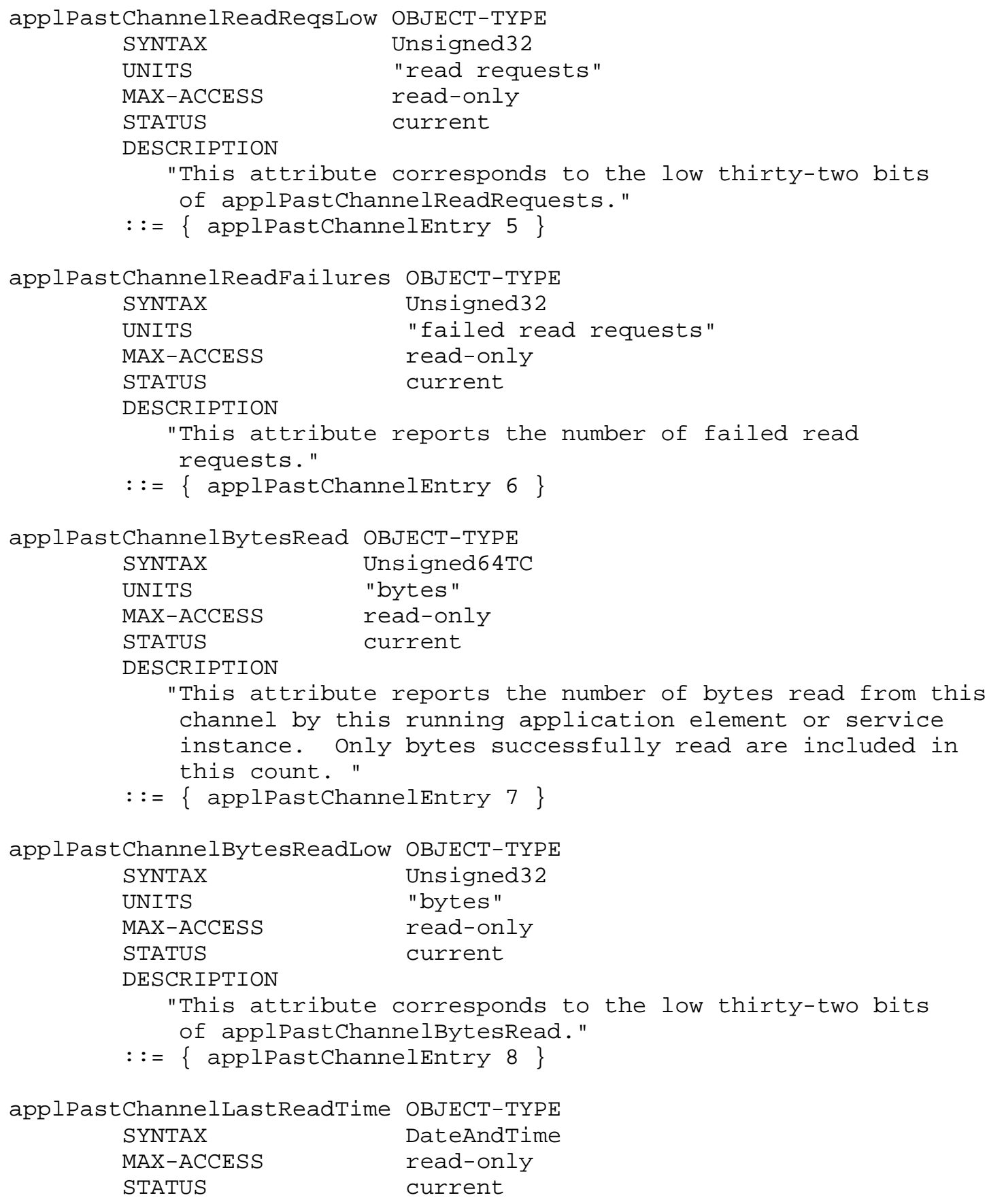

Kalbfleisch, et al. Standards Track [Page 53] 


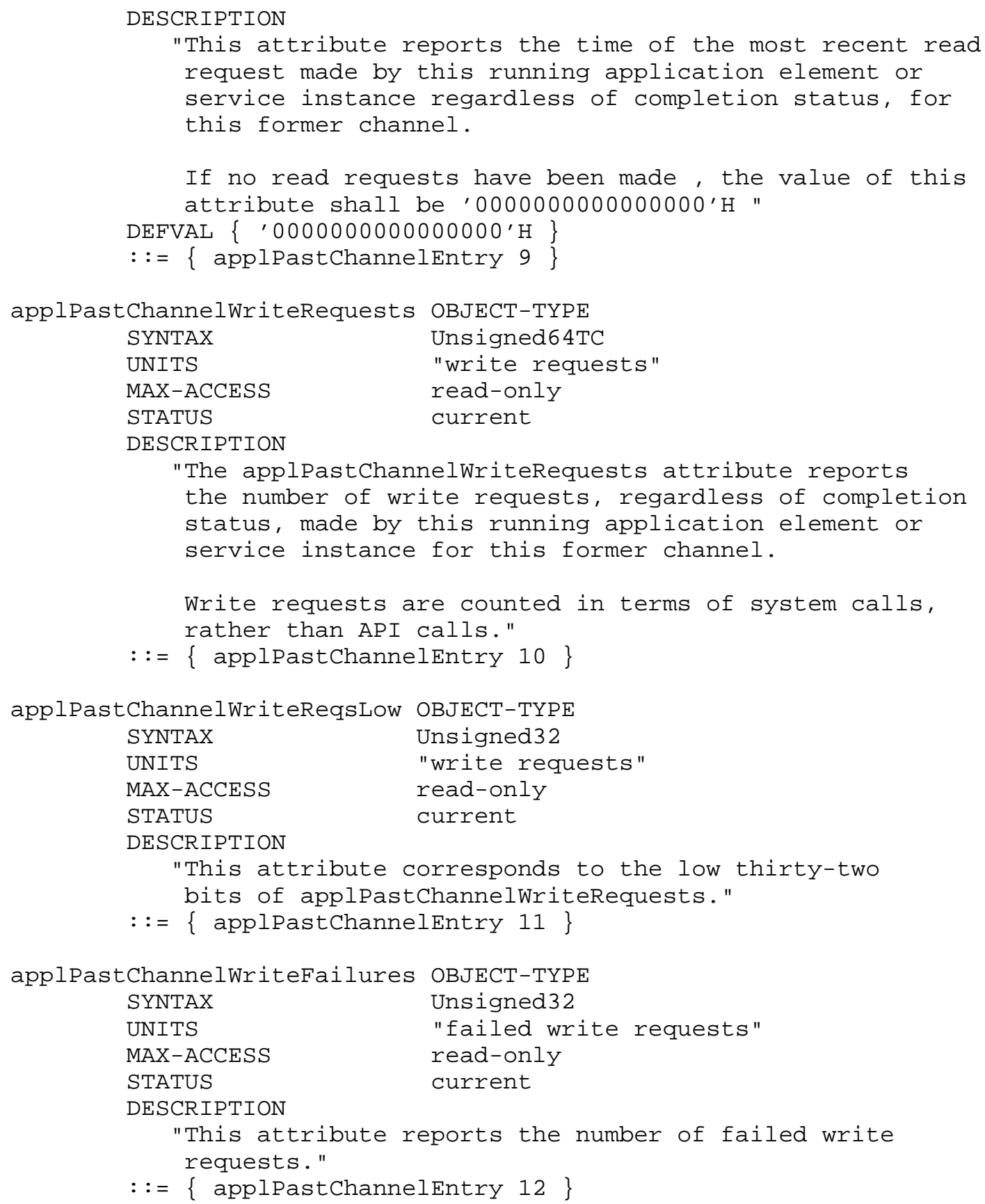




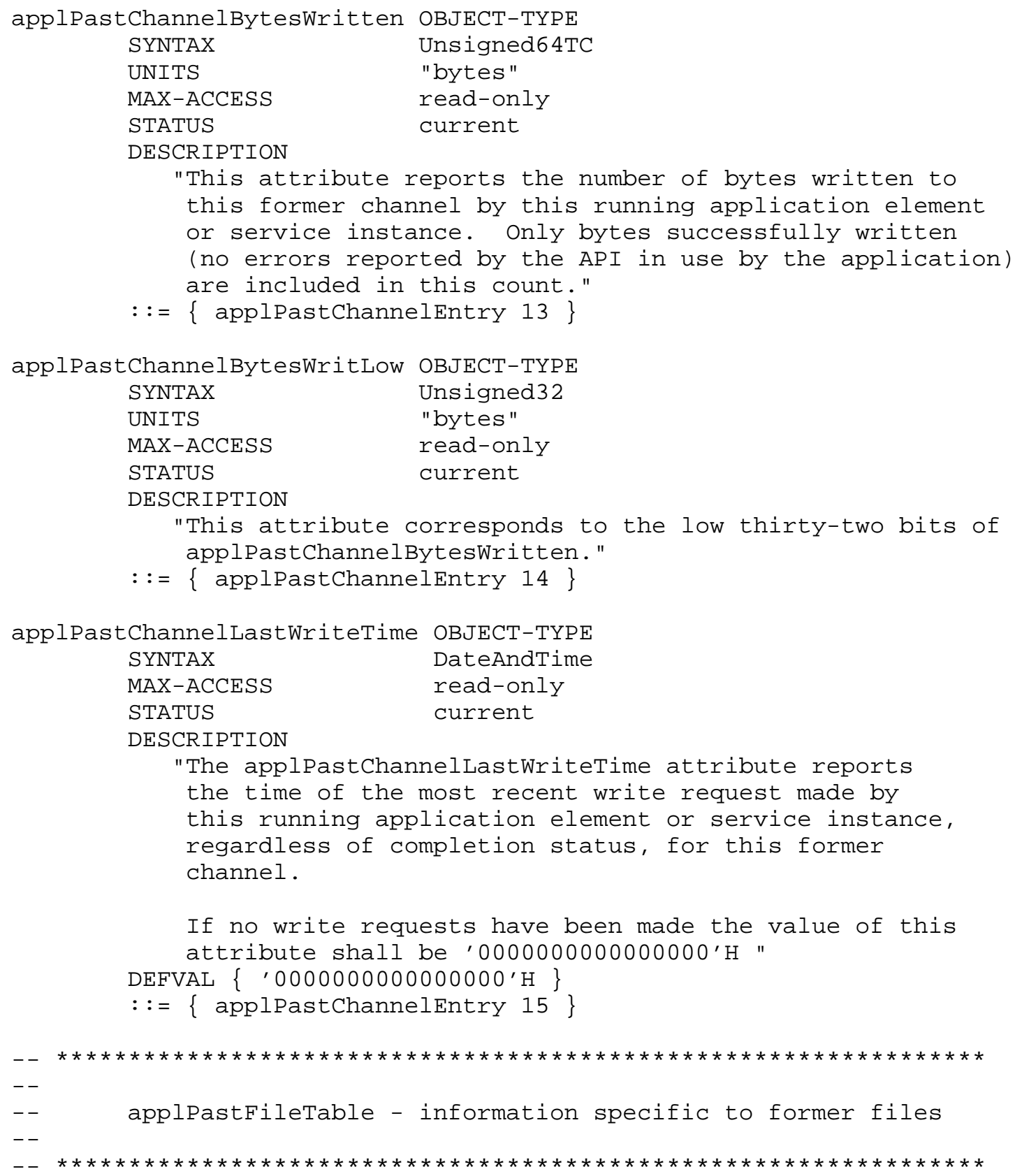




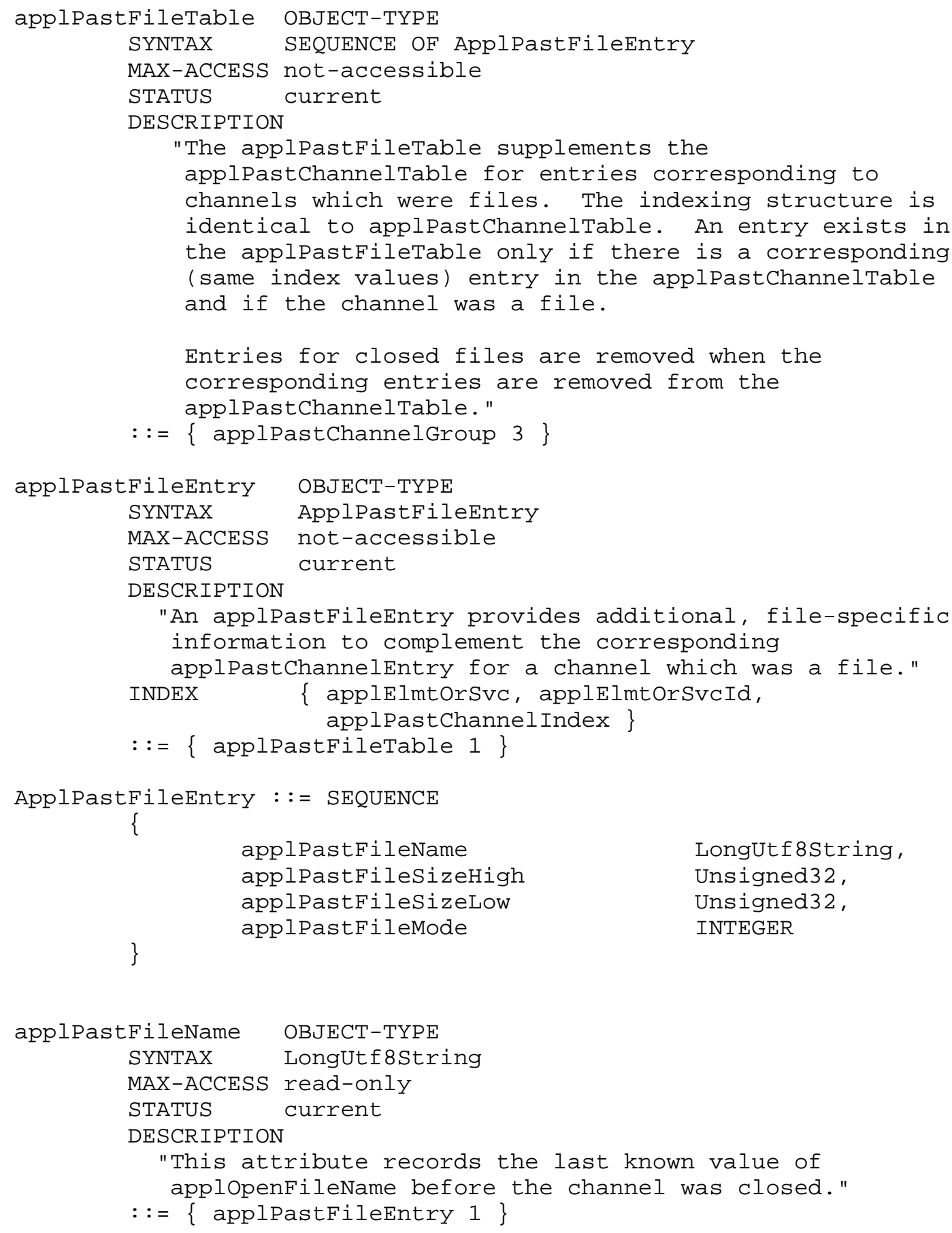




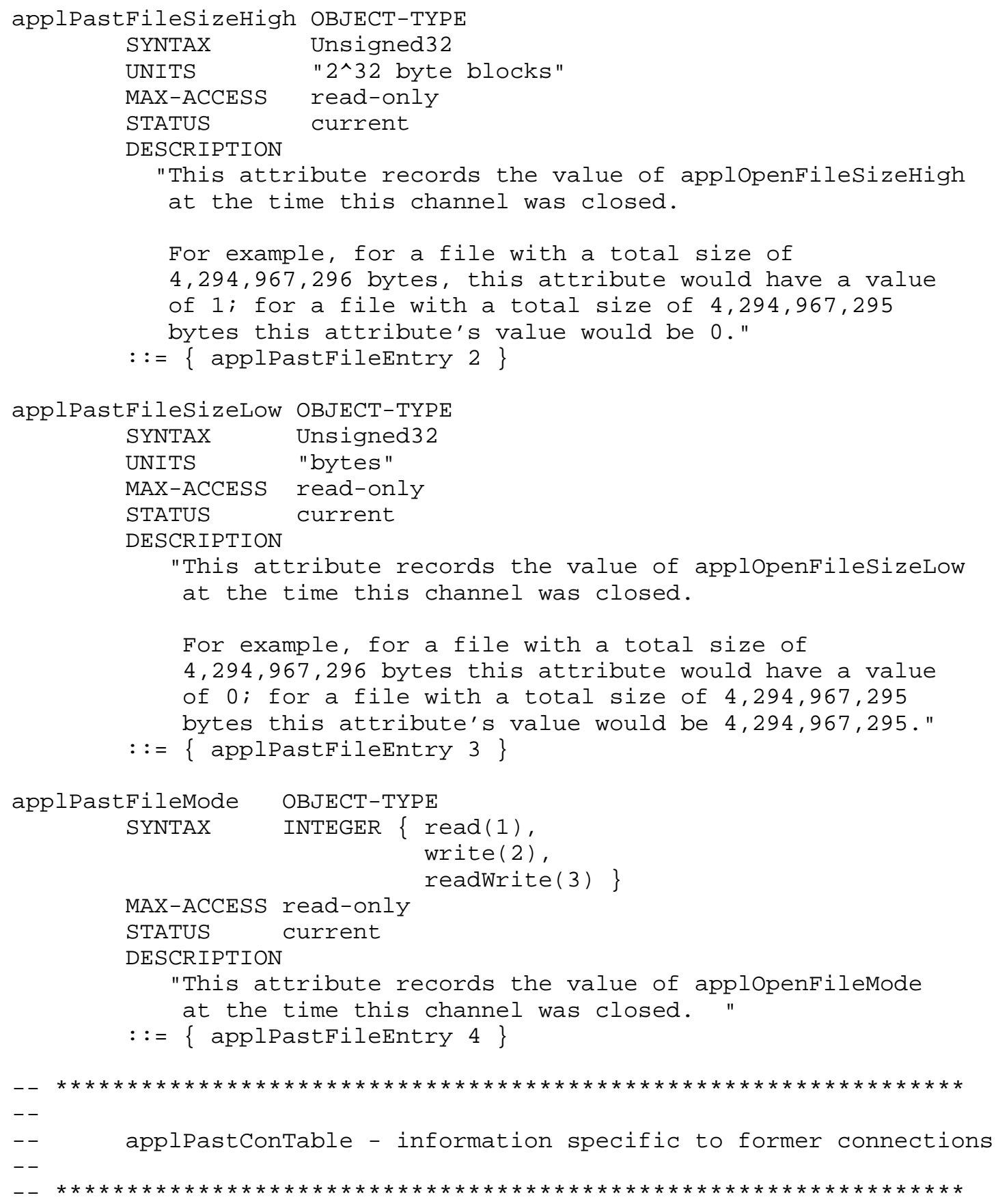




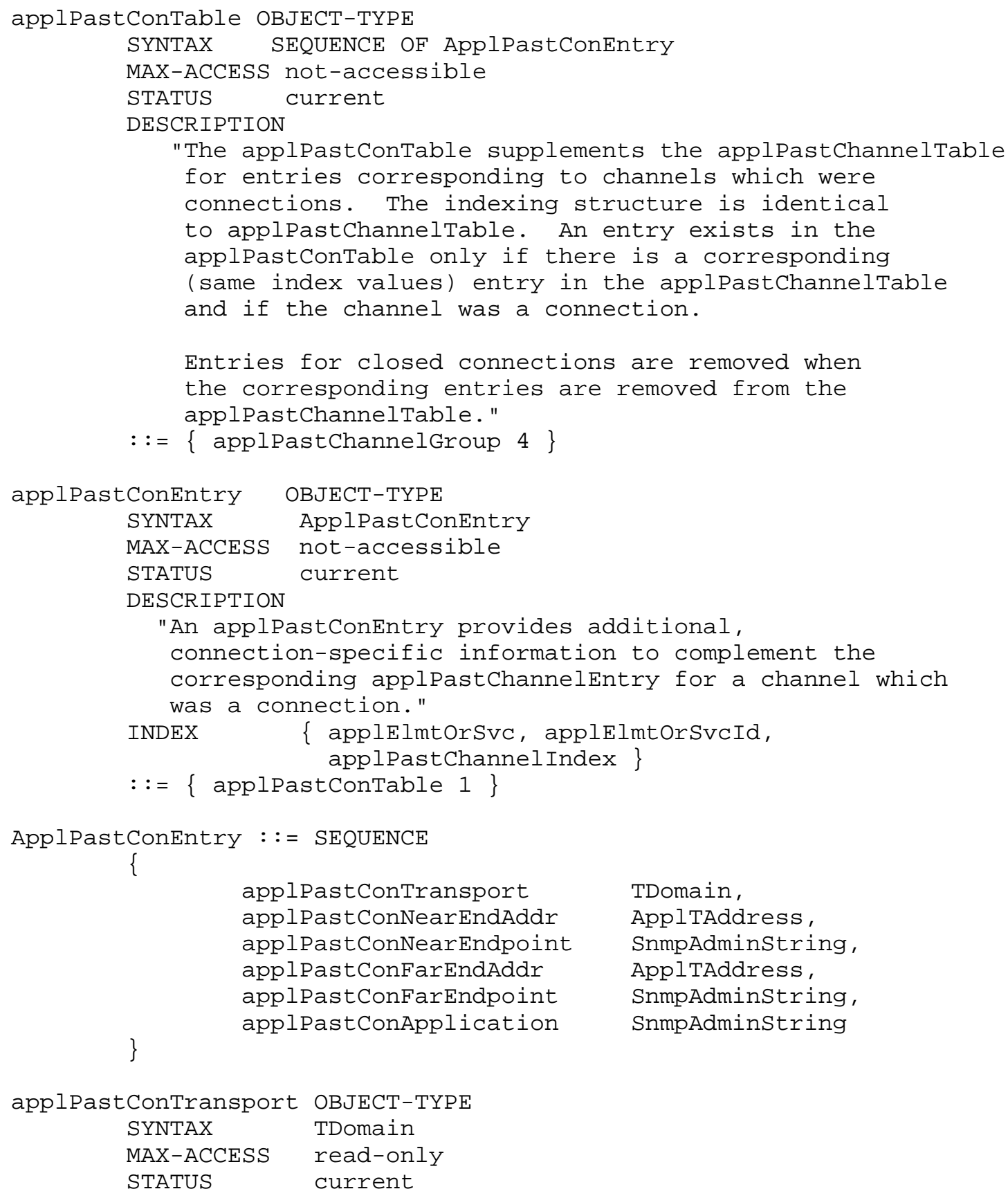




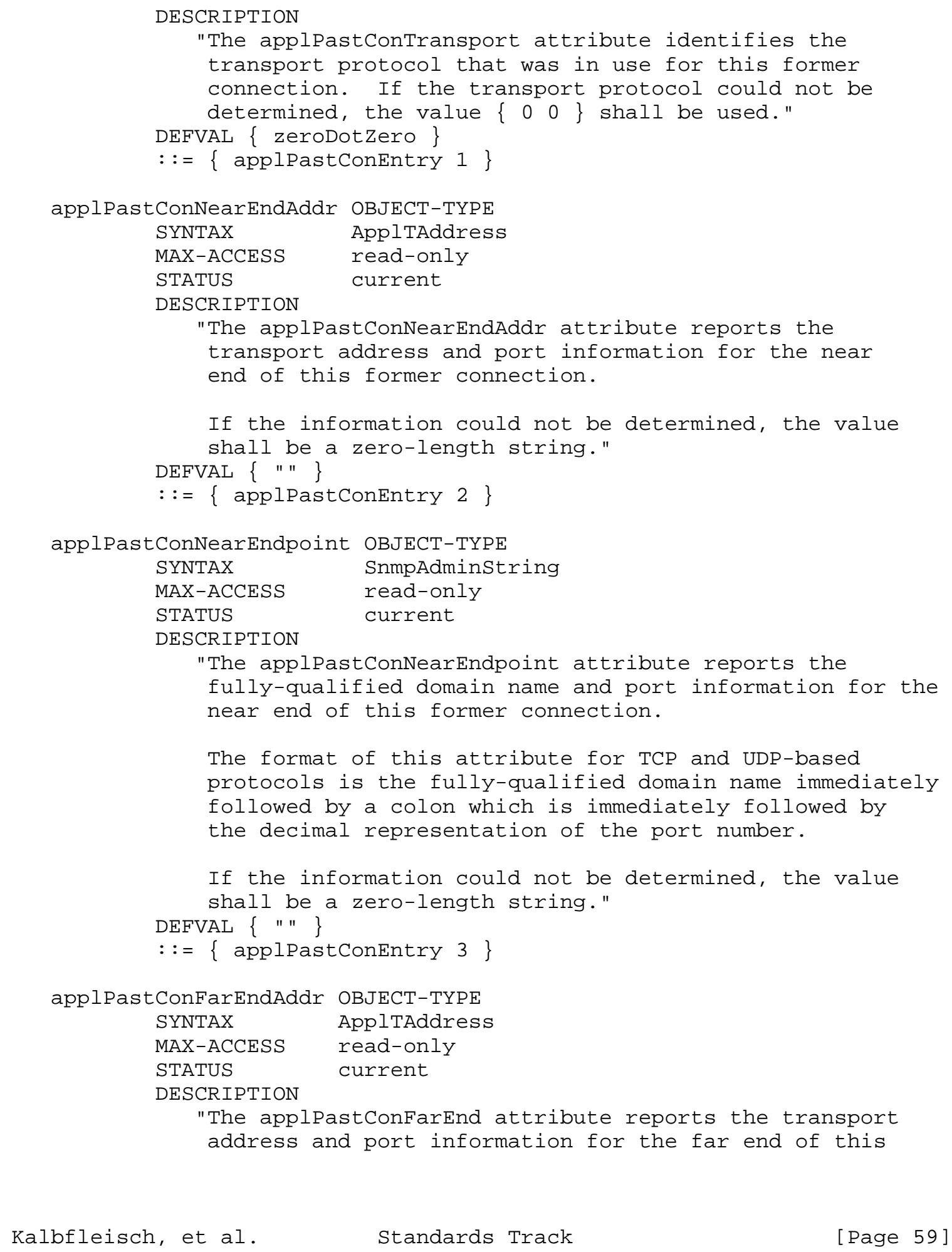









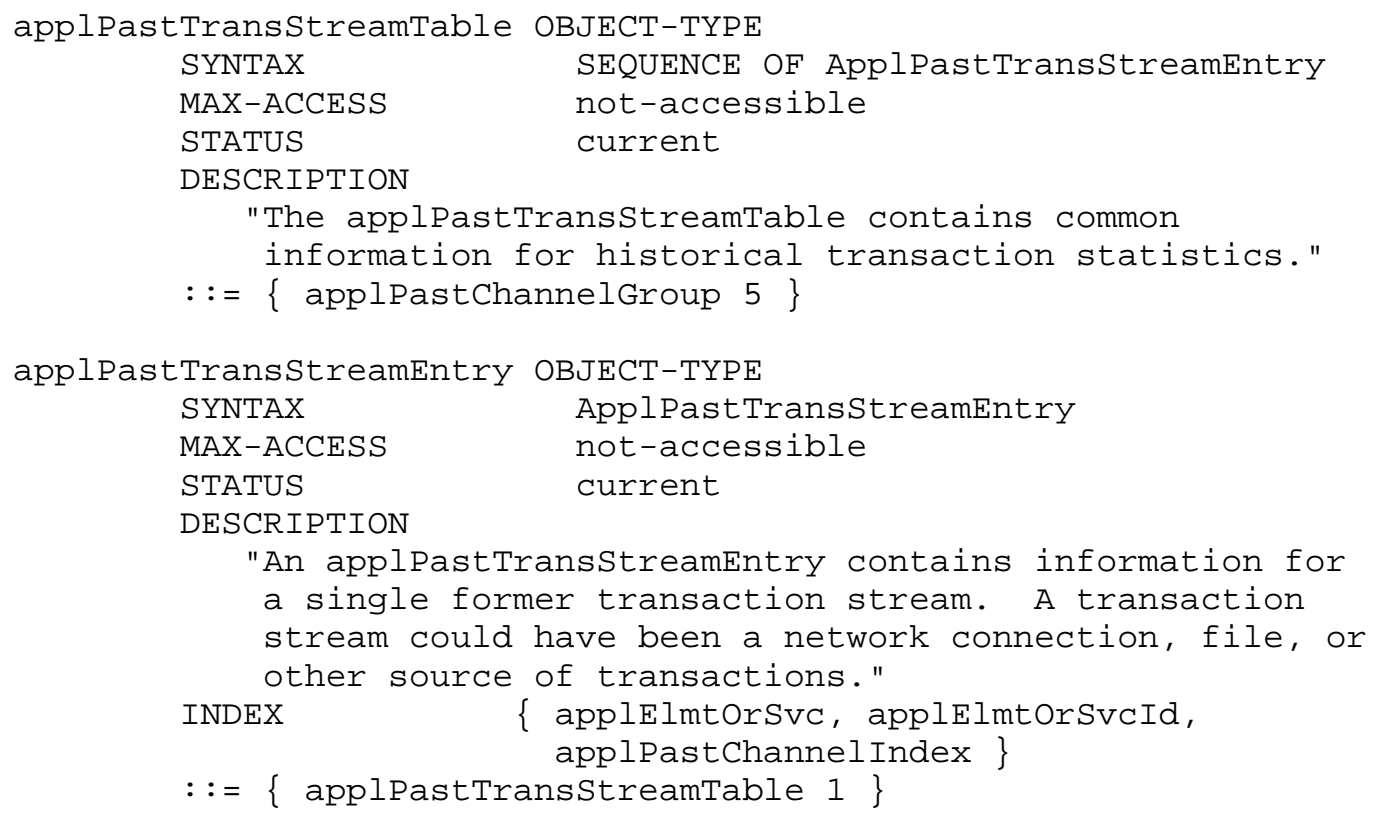




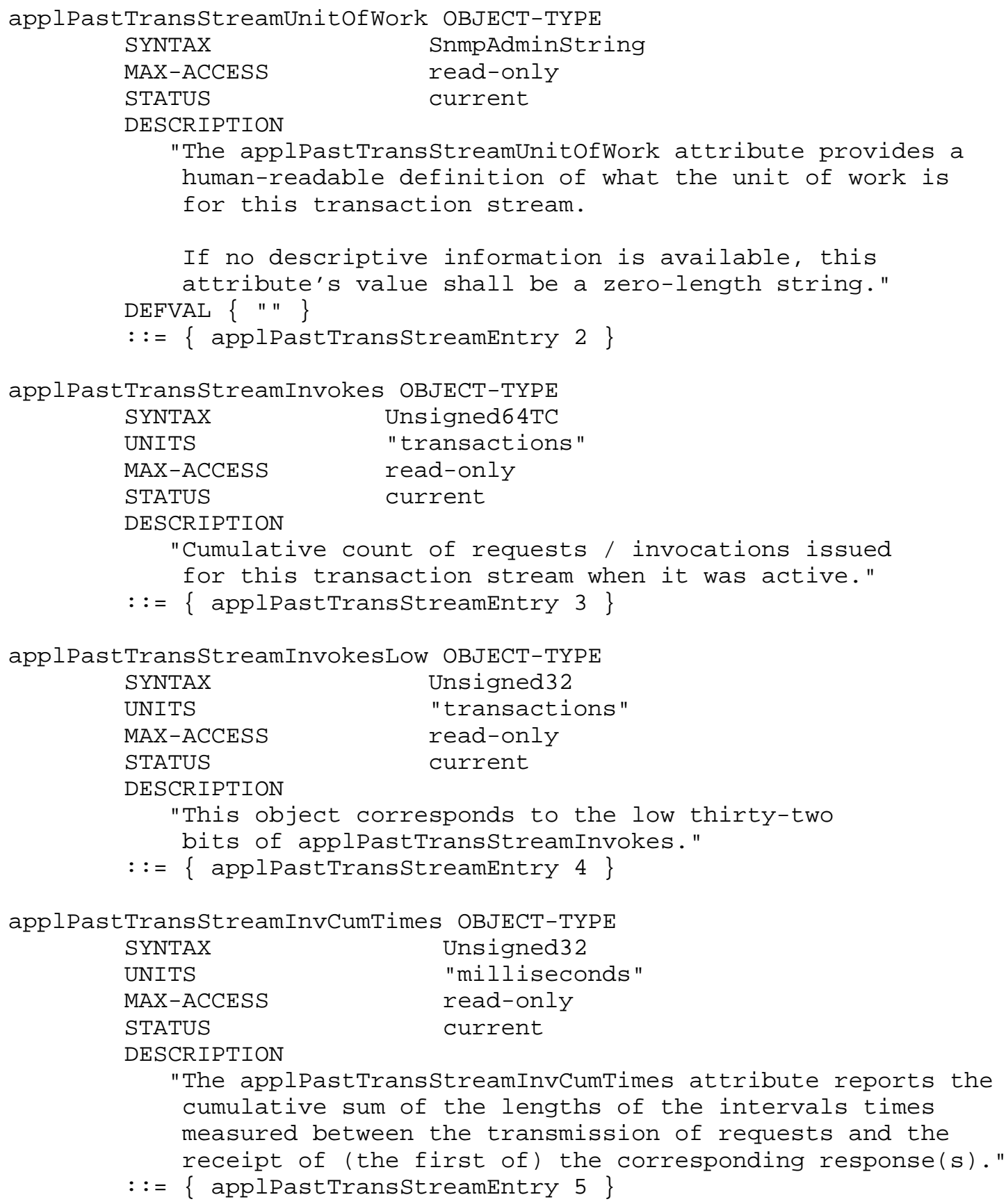




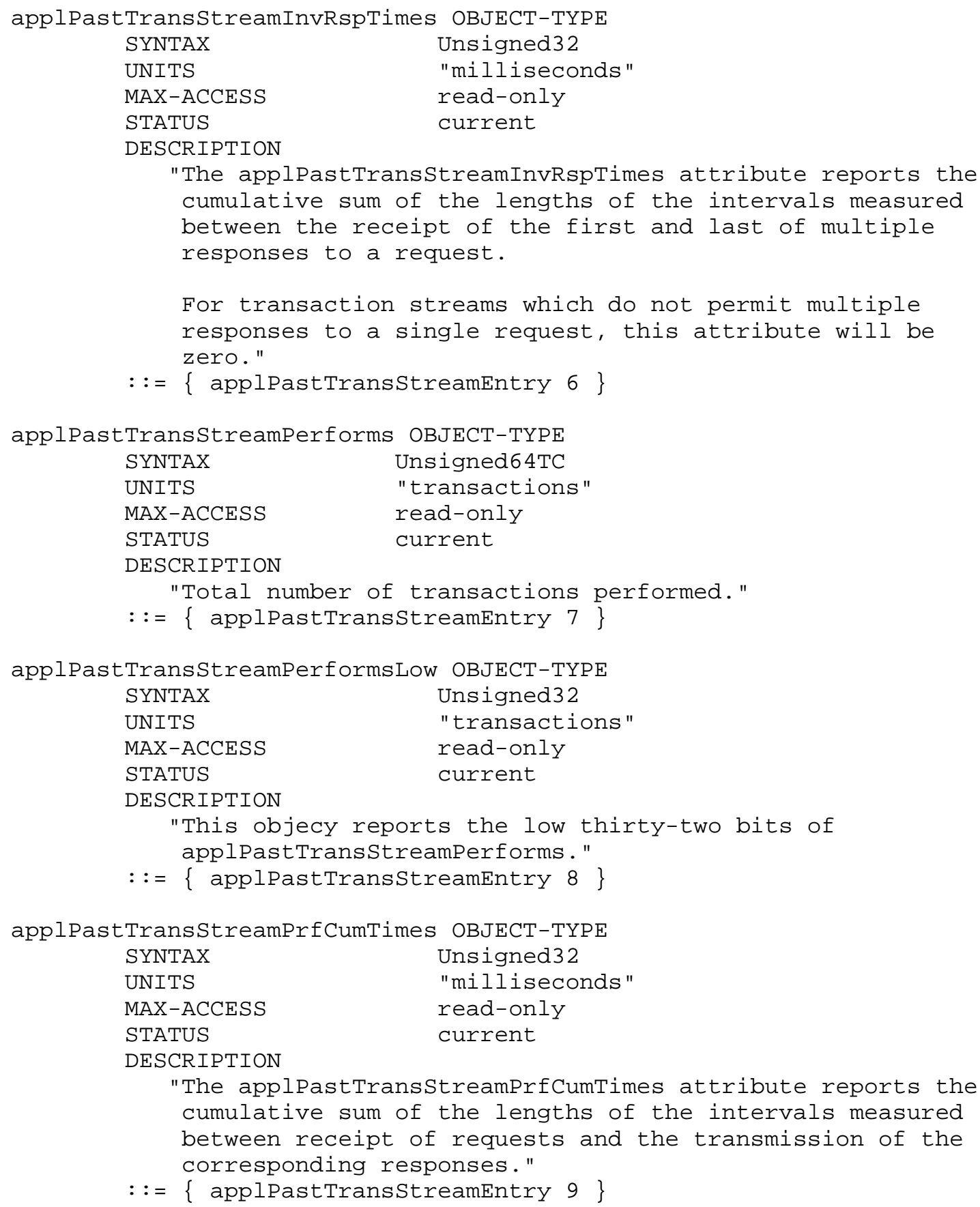

Kalbfleisch, et al.

Standards Track

[Page 63] 


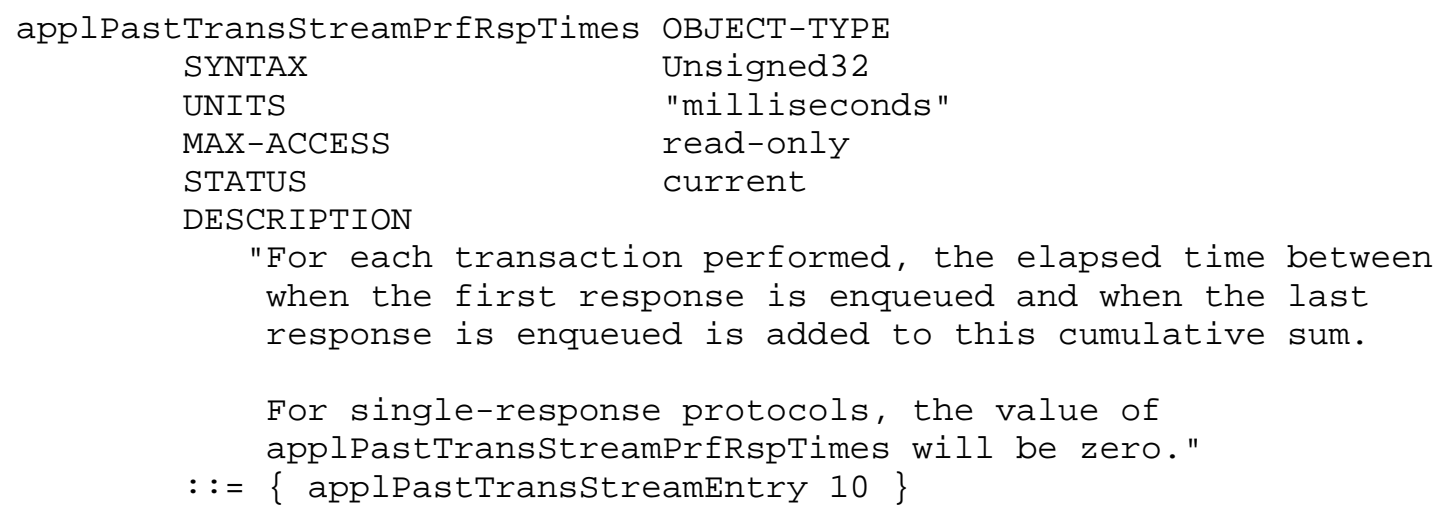

Kalbfleisch, et al.

Standards Track 


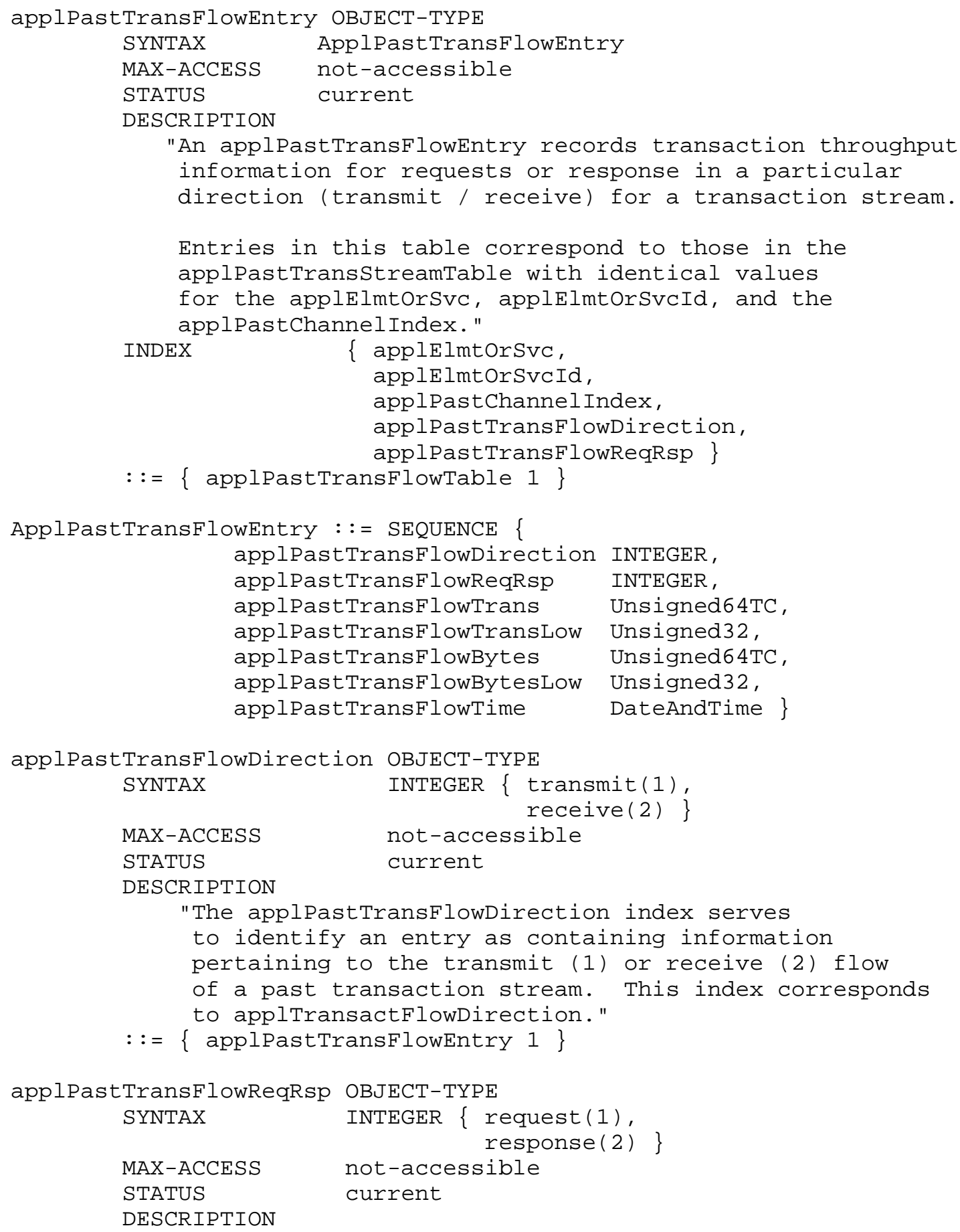

Kalbfleisch, et al.

Standards Track

[Page 65] 


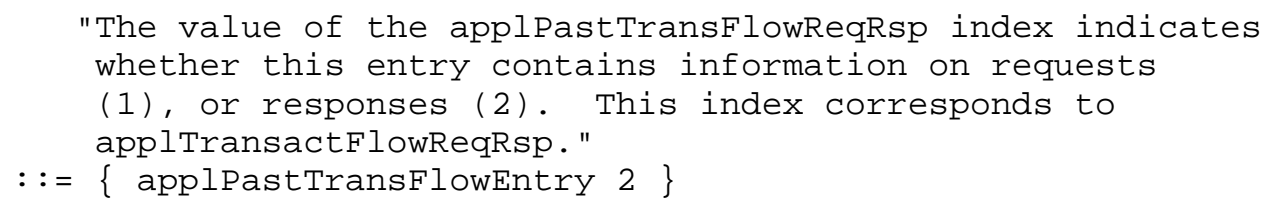

applPastTransFlowTrans OBJECT-TYPE

SYNTAX Unsigned64TC

UNITS "transactions"

MAX-ACCESS read-only

STATUS current

DESCRIPTION

"The applpastTransFlowTrans attribute reports the number of request/response (as indicated by the applPast TransFlowRegRsp index) transactions received/generated (as indicated by the applpast TransflowDirection index) handled on this transaction stream."

$::=\{$ applpastTransflowEntry 3$\}$

applPastTransFlowTransLow OBJECT-TYPE

SYNTAX Unsigned32

UNITS "transactions"

MAX-ACCESS read-only

STATUS current

DESCRIPTION

"This attribute corresponds to the low thirty-two

bits of applpastTransFlowTrans."

$::=\{$ applPastTransflowEntry 4$\}$

$\begin{array}{cl}\text { applPastTransFlowBytes } & \text { OBJECT-TYPE } \\ \text { SYNTAX } & \text { Unsigned64TC } \\ \text { UNITS } & \text { "bytes" } \\ \text { MAX-ACCESS } & \text { read-only } \\ \text { STATUS } & \text { current } \\ \text { DESCRIPTION } & \end{array}$

DESCRIPTION

"The applpastTransflowBytes attribute reports the number of request/response (as indicated by the applPastTransFlowReqRsp index) bytes received/generated (as indicated by the applpastTransFlowDirection index) handled on this transaction stream.

All application layer bytes are included in this count, including any application layer wrappers, headers, or other overhead."

$::=\{$ applpastTransFlowEntry 5$\}$ 


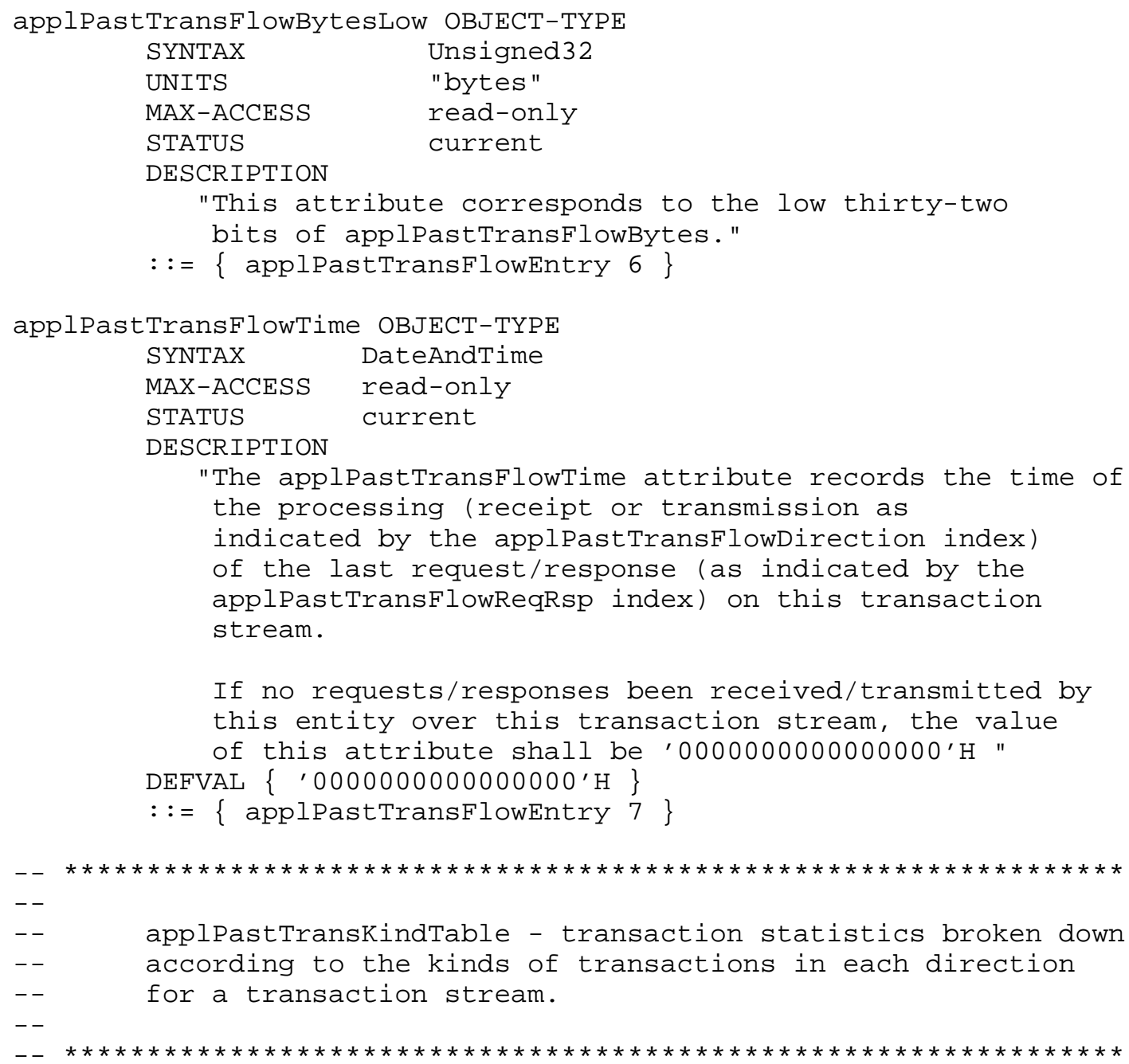




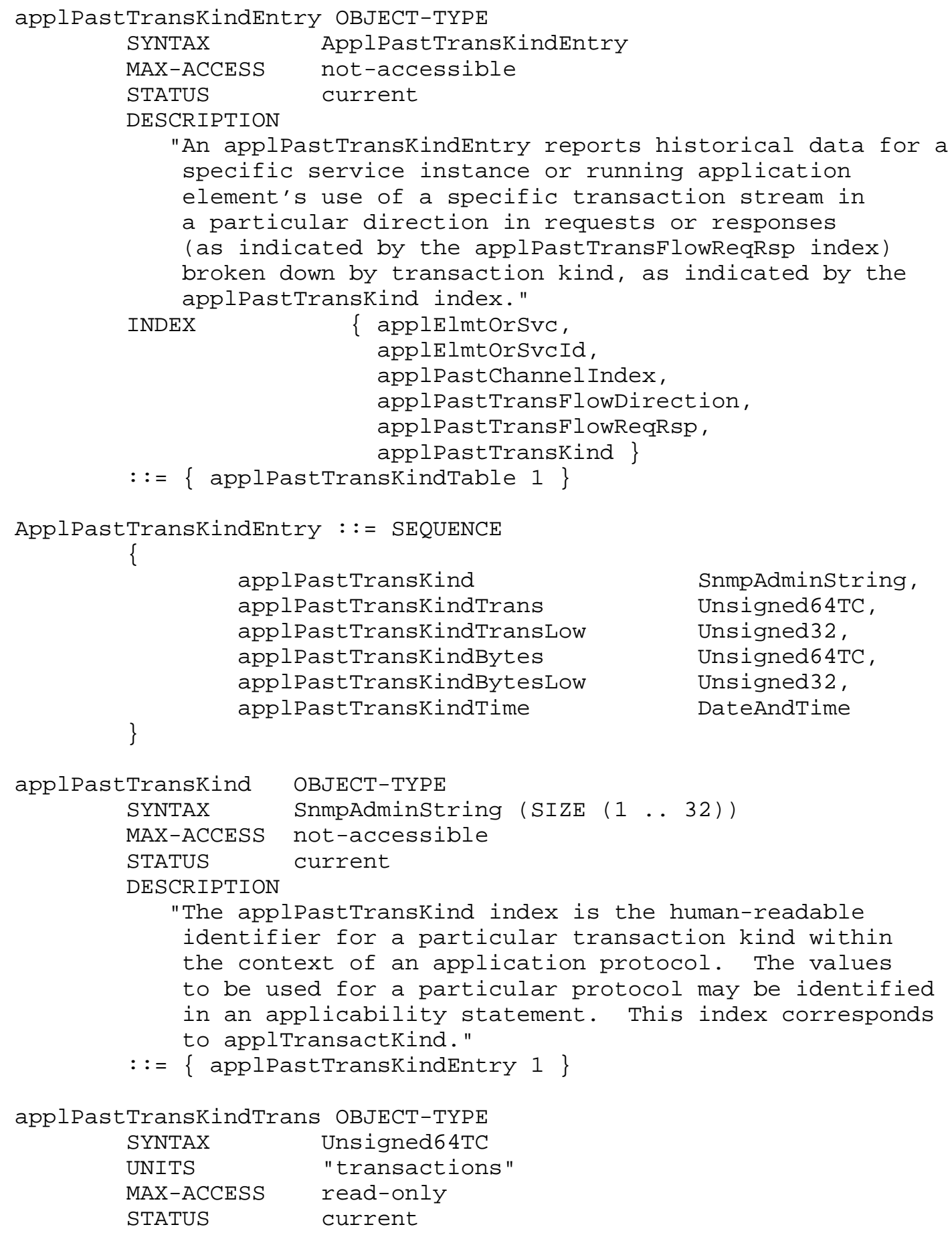




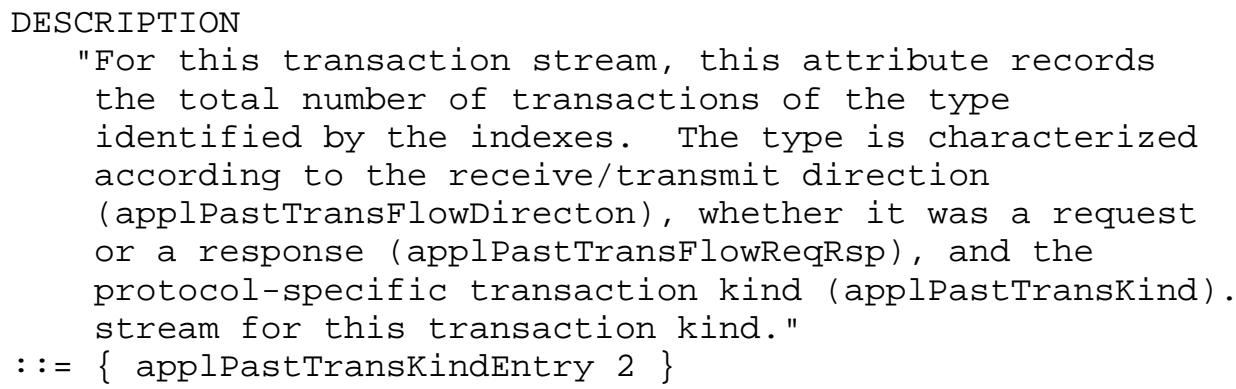

All application layer bytes are included in this count, including any application layer wrappers, headers, or other overhead."

$::=\{$ applpastTranskindEntry 4$\}$

applPastTranskindBytesLow OBJECT-TYPE

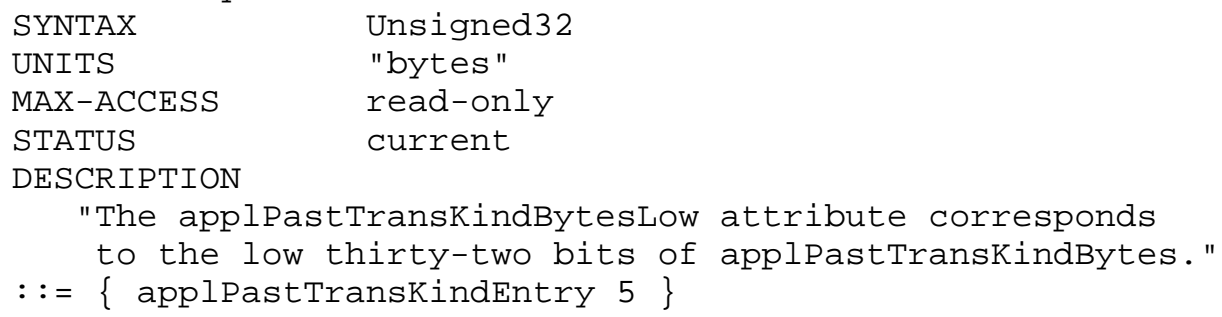




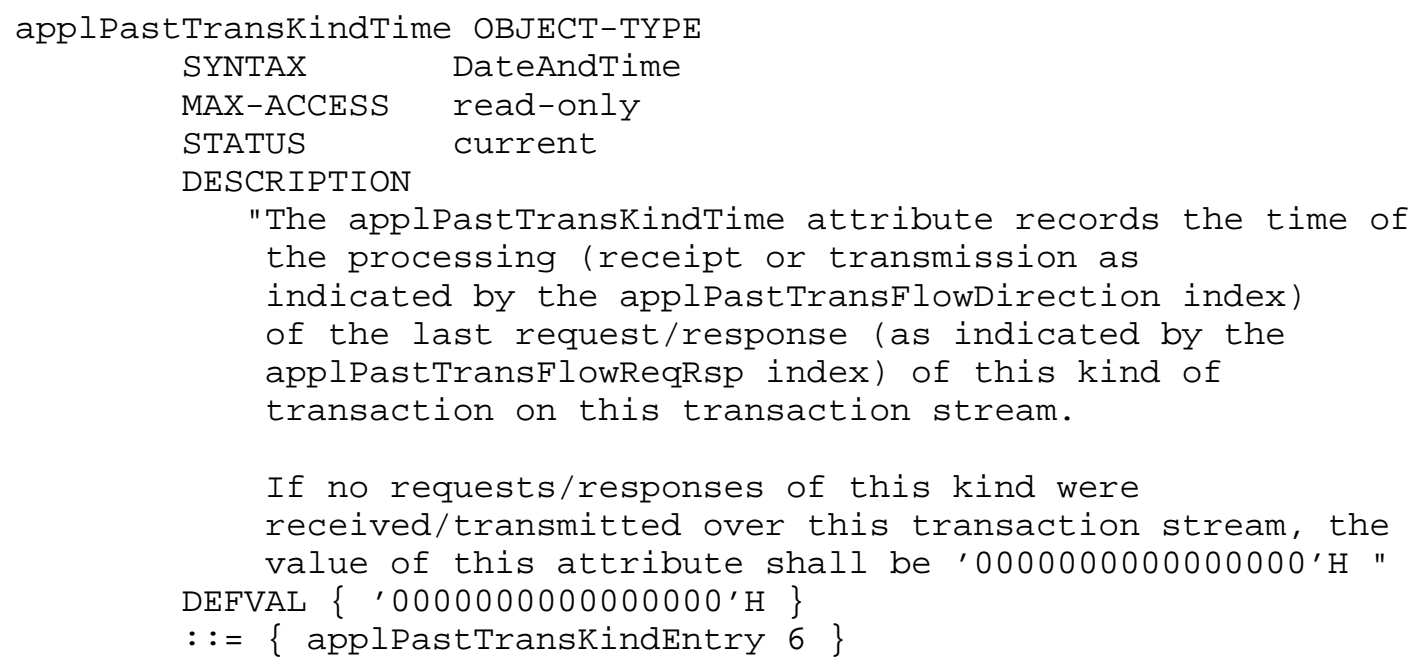

"An applElmtRunstatusEntry contains information to support the control and monitoring of a single running application element."

INDEX \{ sysApplElmtRunIndex \}

$::=\{$ applelmtRunstatusTable 1$\}$ 


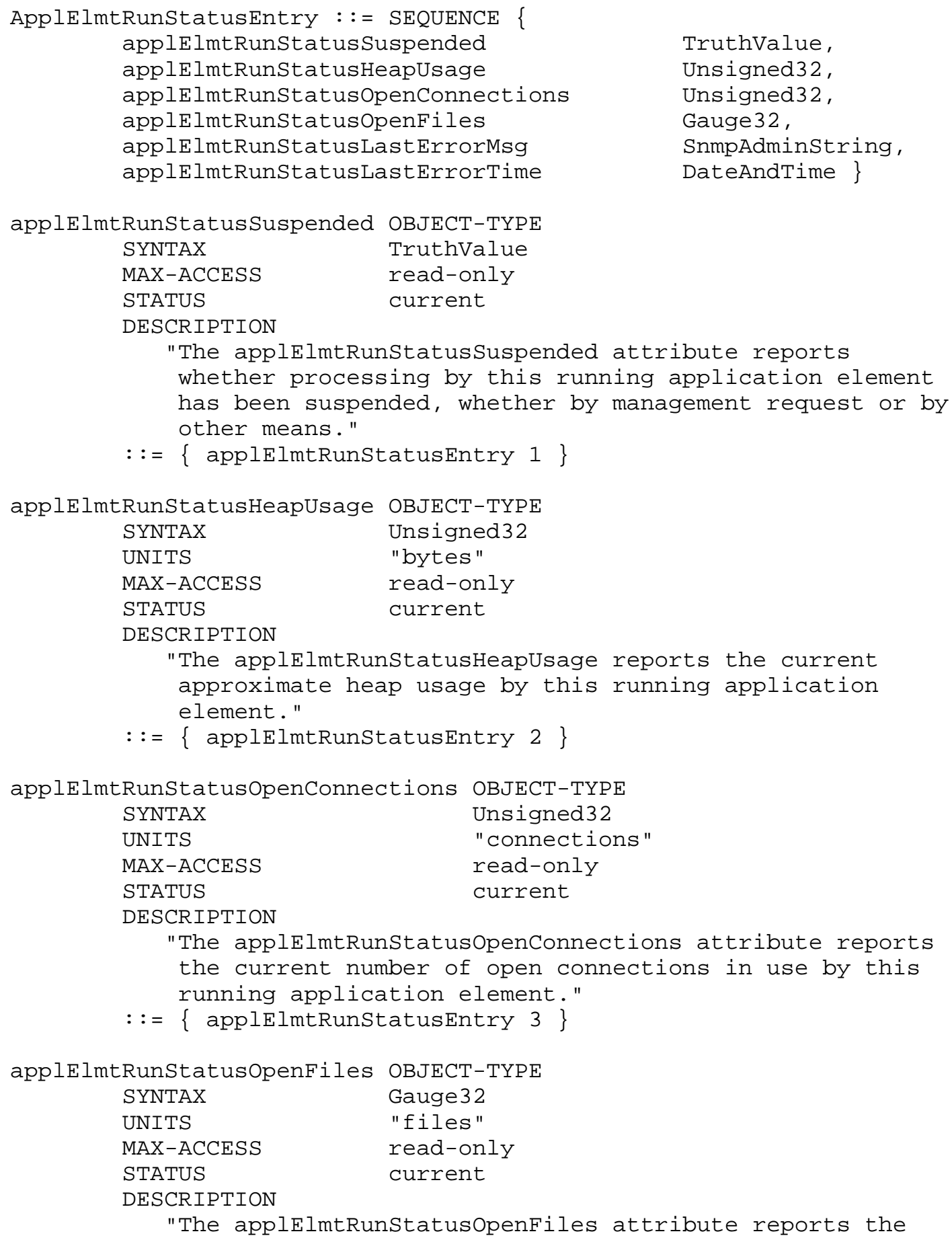




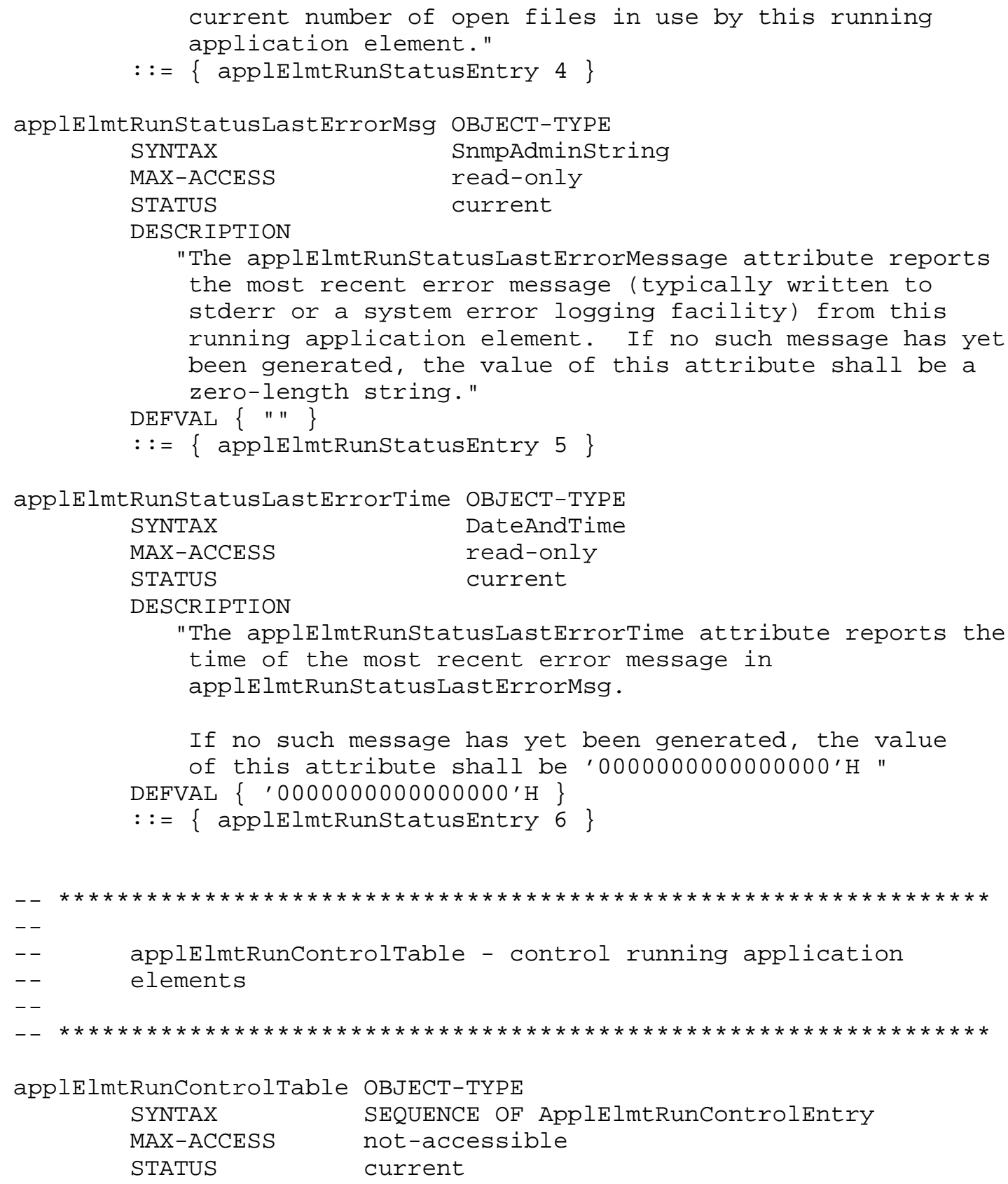

Kalbfleisch, et al. Standards Track [Page 72] 


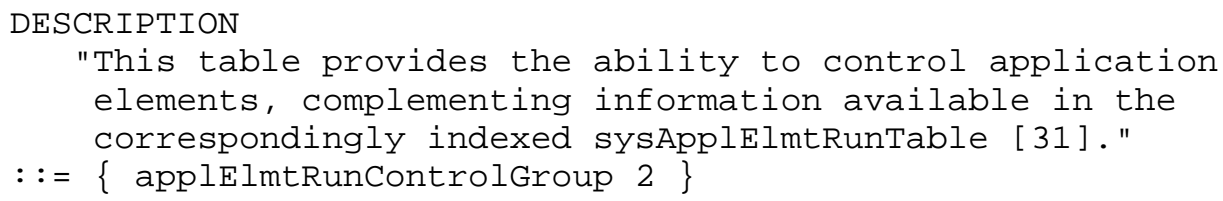




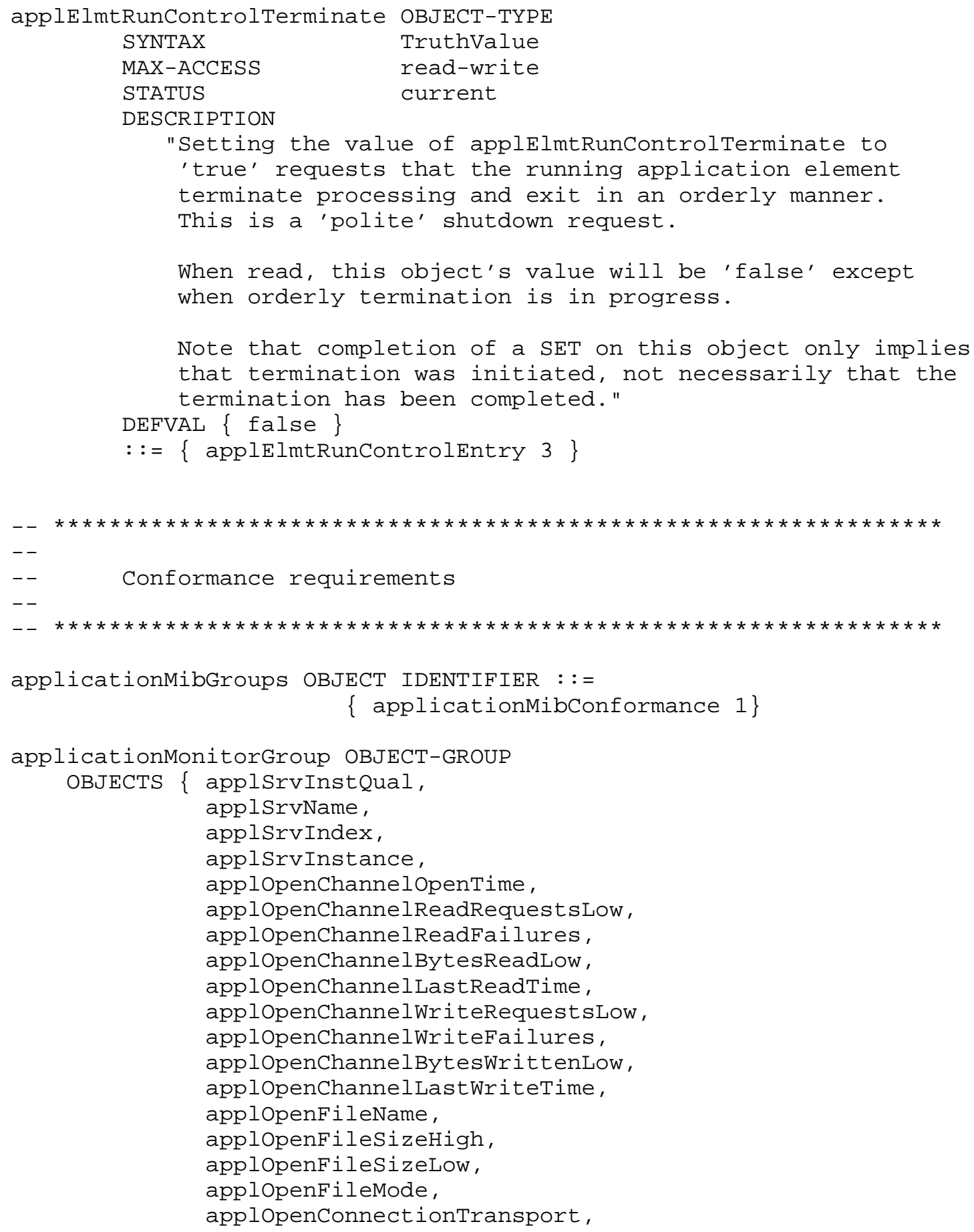




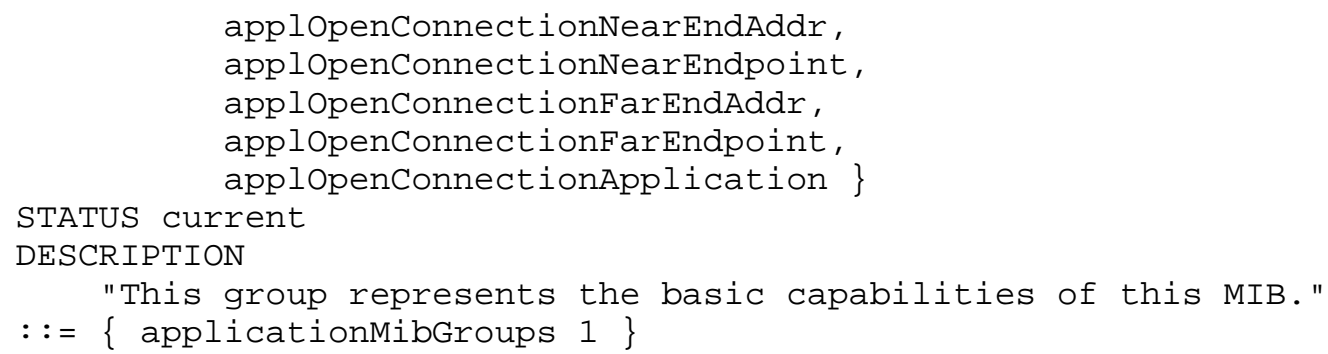

Kalbfleisch, et al.

Standards Track

[Page 75] 


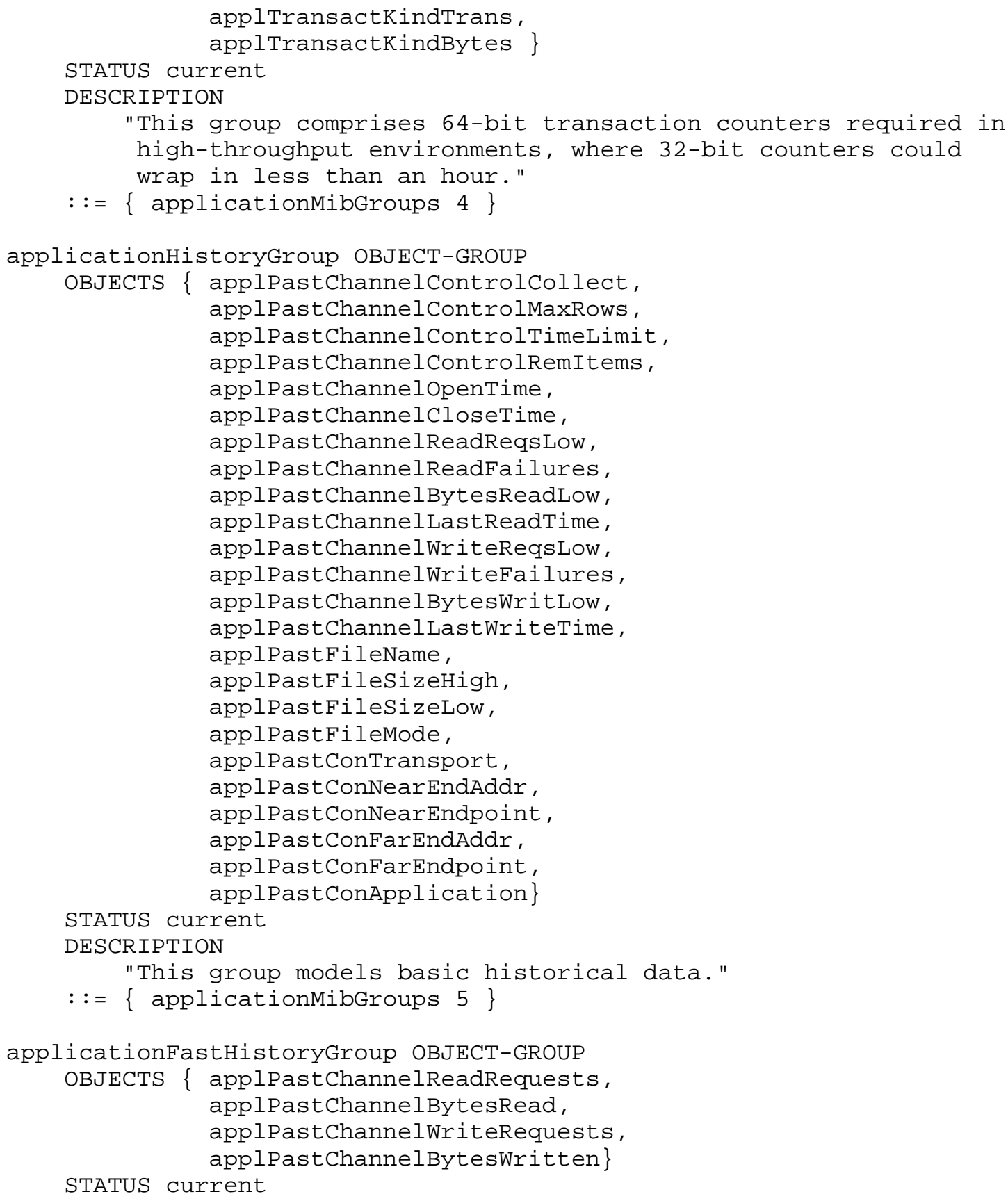




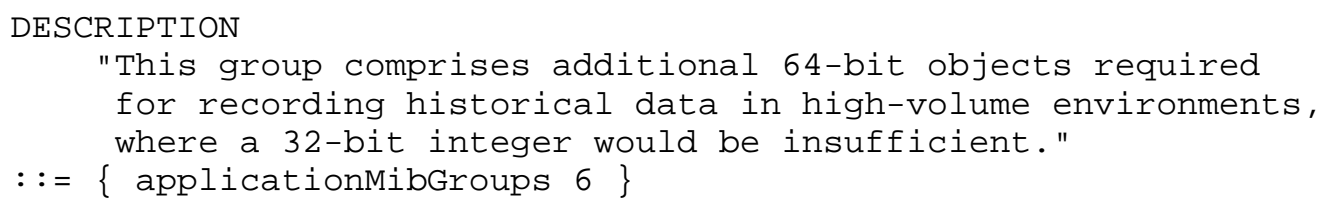

applicationFastTransHistoryGroup OBJECT-GROUP

OBJECTS \{ applPastTransFlowTrans, applPastTransflowBytes, applPastTranskindTrans, applPastTranskindBytes, applpast Trans Streamperforms, applPastTransStreamInvokes \}

STATUS current

DESCRIPTION

"This group contains 64-bit objects required for historical records on high-volume transaction-structured streams, where 32-bit integers would be insufficient." $::=\{$ applicationMibGroups 8 \}

applicationRunGroup OBJECT-GROUP

OBJECTS \{ applElmtRunStatusSuspended, applElmtRunStatusHeapUsage, applElmtRunstatusOpenConnections, applelmtRunstatusOpenfiles, applElmtRunStatusLastErrorMsg, applElmtRunStatusLastErrorTime, 
STATUS current

applelmtRunControlsuspend, applelmtRunControlReconfigure, applelmtRunControlTerminate \}

DESCRIPTION

"This group represents extensions to the system application MIB. "

$::=\{$ applicationMibGroups 9$\}$

applicationMibcompliance MODULE-COMPLIANCE

STATUS current DESCRIPTION "The compliance statement for the application MIB." MODULE

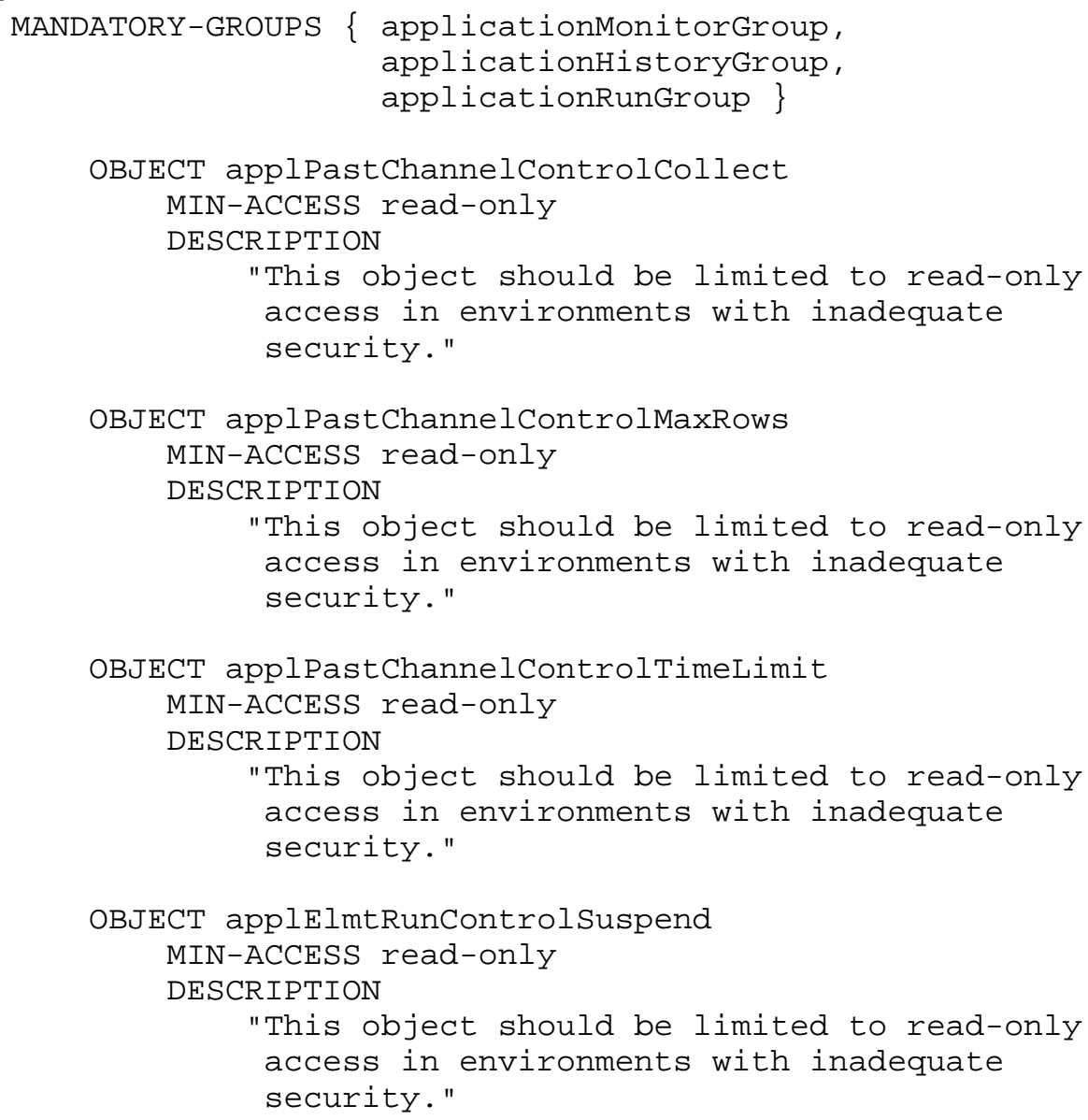




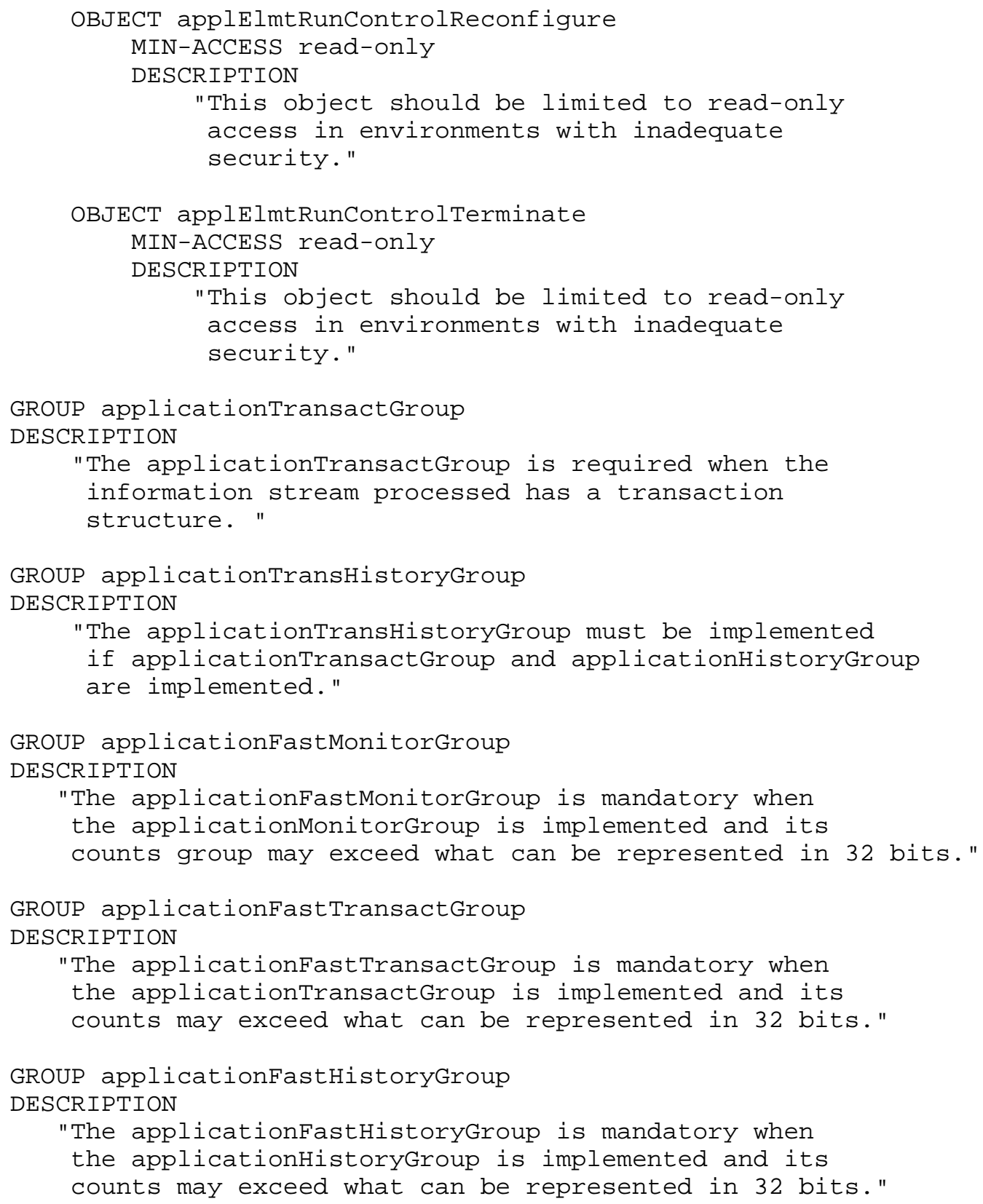




\section{GROUP applicationFastTransHistoryGroup DESCRIPTION \\ "The applicationfastTransHistoryGroup is mandatory when the applicationTransHistoryGroup is implemented and its counts may exceed what can be represented in 32 bits." $::=\{$ applicationMibConformance 2 \}}

END

6. Implementation Issues

Unlike the system application MIB [31], in many environments support for much of this MIB requires instrumentation built into the managed resource. Some tables may be implemented by a single monitor process; for others, the implementation may be distributed within the managed system with the resources being managed.

As a practical matter, this means that the management infrastructure of the managed system must support different subagents taking responsibility for different rows of a single table. This can be supported by Agentx [25], as well as some other subagent protocols such as [8], [9], and [11].

The sysApplRunElmtIndex is the key connection between this MIB and the systems application MIB. Implementations of these two MIBs intended to run concurrently on a given platform must employ a consistent policy for assigning this value to running application elements.

Some of the objects defined in this MIB may carry a high run-time cost in some environments. For example, tracking transaction elapsed time could be expensive if it required two kernel calls (start and finish) per transaction. Similarly, maintaining tables of pertransaction information, rather than aggregating information by transaction type or transaction stream, could have significant storage and performance impacts.

Unless a collision-free mechanism for allocating service instance indexes is in place, the structure of the service-level tables makes an index-reservation mechanism necessary. Agentx [25] is an example of a subagent protocol capable of satisfying this requirement.

7. Intellectual Property

The IETF takes no position regarding the validity or scope of any intellectual property or other rights that might be claimed to pertain to the implementation or use of the technology described in 
this document or the extent to which any license under such rights might or might not be available; neither does it represent that it has made any effort to identify any such rights. Information on the IETF's procedures with respect to rights in standards-track and standards-related documentation can be found in BCP-11. Copies of claims of rights made available for publication and any assurances of licenses to be made available, or the result of an attempt made to obtain a general license or permission for the use of such proprietary rights by implementors or users of this specification can be obtained from the IETF Secretariat.

The IETF invites any interested party to bring to its attention any copyrights, patents or patent applications, or other proprietary rights which may cover technology that may be required to practice this standard. Please address the information to the IETF Executive Director.

\section{Acknowledgements}

This document was produced by the Application MIB working group.

The editor gratefully acknowledges the comments and contributions of the following individuals:

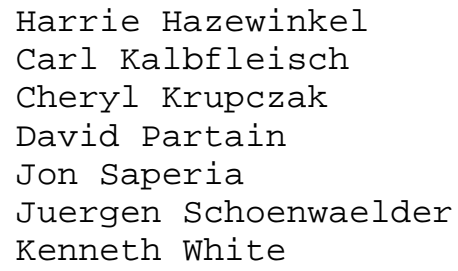

9. Security Considerations

By making potentially sensitive information externally accessible, the capabilities supported by the MIB have the potential of becoming security problems. How security fits into SNMP frameworks is described in [26], and a specific access control model is described in $[30]$.

The tables in this MIB are organized to separate sensitive control capabilities from less sensitive usage information. For example, the objects to control application suspend/resume are separated from those to handle reconfiguration, which in turn are distinct from those for termination. This recognizes the need to support configurations where the level of authorization needed by a manager to do a "reconfigure" might be substantially less than the level needed to terminate an application element. By keeping these in 
separate columns, we make it possible to set up access control that allows, for example, "reconfigure" but not "kill".

The MIB is structured to be useful for managers with read-only access rights. In some environments, it may be approprate to restrict even read-only access to these MIBs.

The capabilities supported by this MIB include several that may be of value to a security administrator. These include the ability to monitor the level of usage of a given application, and to check the integrity of application components.

10. References

[1] ARM Working Group, "Application Response Measurement (ARM) API Guide, Version 2", September, 1997.

[2] IEEE P1387.2, POSIX System Administration - Part 2: Software Administration. (Draft)

[3] ITU-T Recommendation X.744| ISO/IEC IS 10164-18:1996, Information Technology - Open Systems Interconnection - Systems Management: Software Management Function, 1996.

[4] Rose, M. and K. McCloghrie, "Structure and Identification of Management Information for TCP/IP-based Internets", STD 16, RFC 1155, May 1990.

[5] Case, J., Fedor, M., Schoffstall, M. and J. Davin, "Simple Network Management Protocol", STD 15, RFC 1157, May 1990.

[6] Rose, M. and K. McCloghrie, "Concise MIB Definitions", STD 16, RFC 1212, March 1991.

[7] Rose, M., "A Convention for Defining Traps for use with the SNMP", RFC 1215, March 1991.

[8] Rose, M., "SNMP MUX Protocol and MIB", RFC 1227, May 1991.

[9] Carpenter, G. and B. Wijnen, "SNMP-DPI Simple Network Management Protocol Distributed Program Interface", RFC 1228, May 1991.

[10] Grillo, P. and S. Waldbusser, "Host Resources MIB", RFC 1514, September 1993 .

[11] Carpenter, G., Curran, K., Sehgal, A., Waters, G. and B. Wijnen, "Simple Network Management Protocol Distributed Protocol Interface Version 2.0", RFC 1592, March 1994. 
[12] Brower, D., Purvy, R., Daniel, A., Sinykin, M. and J. Smith, "Relational Database Management System (RDBMS) Management Information Base (MIB) using SMIv2", RFC 1697, August 1994.

[13] Reynolds, J. and J. Postel, "Assigned Numbers", STD 2, RFC 1700, October 1994 .

[14] Case, J., McCloghrie, K., Rose, M. and S. Waldbusser, "Introduction to Community-based SNMPv2", RFC 1901, January 1996.

[15] McCloghrie, K., Perkins, D. and J. Schoenwaelder, "Structure of Management Information Version 2 (SMIV2)", STD 58, RFC 2578, April 1999.

[16] McCloghrie, K., Perkins, D. and J. Schoenwaelder, "Textual Conventions for SMIv2", STD 58, RFC 2579, April 1999.

[17] McCloghrie, K., Perkins, D. and J. Schoenwaelder, "Conformance Statements for SMIv2", STD 58, RFC 2580, April 1999.

[18] Case, J., McCloghrie, K., Rose, M. and S. Waldbusser, "Protocol Operations for Version 2 of the Simple Network Management Protocol (SNMPv2)", RFC 1905, January 1996.

[19] Case, J., McCloghrie, K., Rose, M. and S. Waldbusser, "Transport Mappings for Version 2 of the Simple Network Management Protocol (SNMPv2)", RFC 1906, January 1996.

[20] McCloghrie, K. and A. Bierman, "Entity MIB using SMIv2", RFC 2037, October 1996.

[21] Kalbfleisch, C., "Applicability of Standards Track MIBs to Management of World Wide Web Servers", RFC 2039, November 1996.

[22] Bradner, S., "Key words for use in RFCs to Indicate Requirement Levels", BCP 14, RFC 2119, March 1997.

[23] Freed, N. and S. Kille, "Network Services Monitoring MIB", RFC 2248, January 1998 .

[24] Freed, N. and S. Kille, "Mail Monitoring MIB", RFC 2249, January 1998 .

[25] Daniele, M., Francisco, D. and B. Wijnen, "Agent Extensibility (AgentX) Protocol", RFC 2257, January, 1998.

[26] Harrington, D., Presuhn, R. and B. Wijnen, "An Architecture for 
describing SNMP Management Frameworks", RFC 2571, May 1999.

[27] Case, J., Harrington D., Presuhn R. and B. Wijnen, "Message Processing and Dispatching for the Simple Network Management Protocol (SNMP)", RFC 2572, May 1999.

[28] Levi, D., Meyer, P. and B. Stewart, "SNMPv3 Applications", RFC 2573, May 1999.

[29] Blumenthal, U. and B. Wijnen, "User-based Security Model (USM) for version 3 of the Simple Network Management Protocol (SNMPv3)", RFC 2574, May 1999.

[30] Wijnen, B., Presuhn, R. and K. McCloghrie, "View-based Access Control Model for the Simple Network Management Protocol (SNMP) ", RFC 2575, May 1999.

[31] Krupczak, C. and J. Saperia, "Definitions of System-Level Managed Objects for Applications", RFC 2287, February 1998.

11. Authors' Addresses

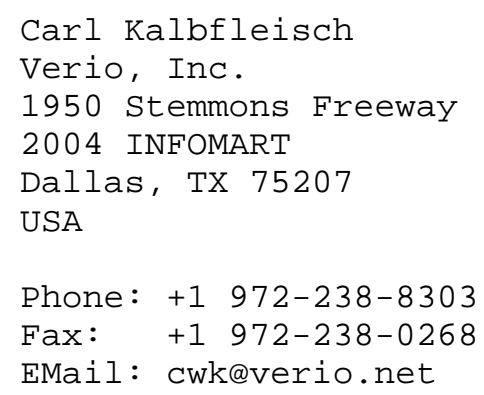




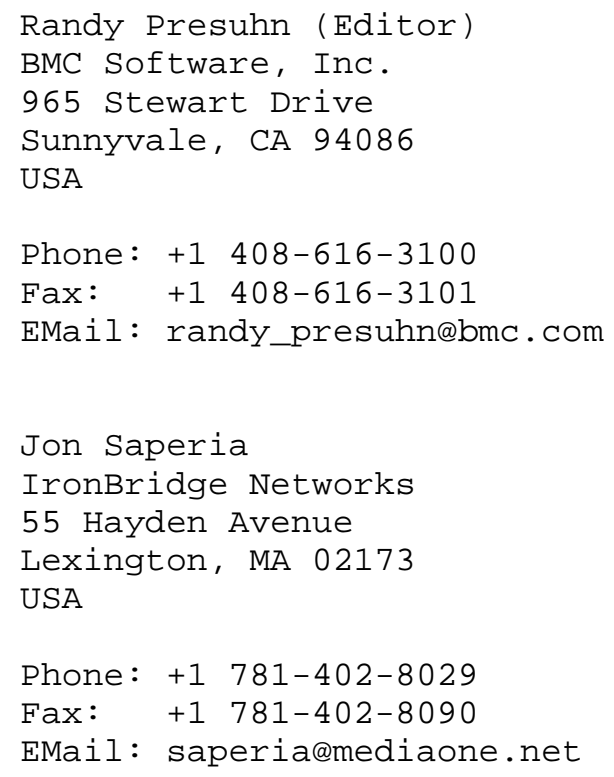


12. Full Copyright statement

Copyright (C) The Internet Society (1999). All Rights Reserved.

This document and translations of it may be copied and furnished to others, and derivative works that comment on or otherwise explain it or assist in its implementation may be prepared, copied, published and distributed, in whole or in part, without restriction of any kind, provided that the above copyright notice and this paragraph are included on all such copies and derivative works. However, this document itself may not be modified in any way, such as by removing the copyright notice or references to the Internet society or other Internet organizations, except as needed for the purpose of developing Internet standards in which case the procedures for copyrights defined in the Internet Standards process must be followed, or as required to translate it into languages other than English.

The limited permissions granted above are perpetual and will not be revoked by the Internet society or its successors or assigns.

This document and the information contained herein is provided on an "AS IS" basis and THE INTERNET SOCIETY AND THE INTERNET ENGINEERING TASK FORCE DISCLAIMS ALL WARRANTIES, EXPRESS OR IMPLIED, INCLUDING BUT NOT LIMITED TO ANY WARRANTY THAT THE USE OF THE INFORMATION HEREIN WILL NOT INFRINGE ANY RIGHTS OR ANY IMPLIED WARRANTIES OF MERCHANTABILITY OR FITNESS FOR A PARTICULAR PURPOSE.

Acknowledgement

Funding for the RFC Editor function is currently provided by the Internet society. 